\title{
HERWIG 6: an event generator for hadron emission reactions with interfering gluons (including supersymmetric processes)*
}

\section{Gennaro Corcella \\ Department of Physics and Astronomy, University of Rochester, USA \\ E-mail: 'corceilia@pas.rochester edư}

\section{Ian G. Knowles}

Department of Physics and Astronomy, University of Edinburgh, UK E-mail: knowles@ph.ed.ac.uk

\section{Giuseppe Marchesini}

Dipartimento di Fisica, Università di Milano-Bicocca, and

I.N.F.N., Sezione di Milano, Italy

E-mail: Giuseppe.Marchesini@mi.infn. ìtín

\section{Stefano Moretti}

Theory Division, CERN, Switzerland

E-mail: 'Stefano.Morettīecern.chi

\section{Kosuke Odagiri}

Theory Group, KEK, Japan

E-mail: odagiriki @post.kek.jpi

\section{Peter Richardson}

Department of Applied Mathematics and Theoretical Physics and

Cavendish Laboratory, University of Cambridge, UK

E-mail: ríchardnohep.phy.cam.ac.uk

\section{Michael H. Seymour}

Rutherford Appleton Laboratory, UK

E-mail: M. Seymour@rì. ac.uk

\section{Bryan R. Webber}

Cavendish Laboratory, University of Cambridge, UK

E-mail: ivebber@hep.phy.cam.ac.uki 
Abstract: HERWIG is a general-purpose Monte Carlo event generator, which includes the simulation of hard lepton-lepton, lepton-hadron and hadron-hadron scattering and soft hadron-hadron collisions in one package. It uses the parton-shower approach for initial- and final-state QCD radiation, including colour coherence effects and azimuthal correlations both within and between jets. This article updates the description of HERWIG published in 1992, emphasising the new features incorporated since then. These include, in particular, the matching of first-order matrix elements with parton showers, a more correct treatment of heavy quark decays, and a wide range of new processes, including many predicted by the Minimal Supersymmetric Standard Model, with the option of R-parity violation. At the same time we offer a brief review of the physics underlying HERWIG, together with details of the input and control parameters and the output data, to provide a self-contained guide for prospective users of the program.

KeYwords: 'Supersymmetric Standard Model, QCD

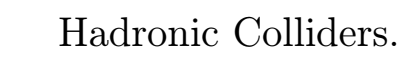

\footnotetext{
${ }^{*}$ Work supported in part by the U.K. Particle Physics and Astronomy Research Council, by the italian Ministero della Università e Ricerca Scientifica, and by the E.U. Fourth Framework Programme 'Training and Mobility of Researchers', Network 'Quantum Chromodynamics and the Deep Structure of Elementary Particles', contract FMRX-CT98-0194 (DG 12 - MIHT). Research of G.C. supported by grant number DE-FG02-91ER40685 from the U.S. Department of Energy.
} 


\section{Contents}

ii. Introduction

2. Using HERWIG

3. Physics simulated by HERWIG 6

13.1 Elementary hard subprocess

13.2 Parton showers

P. $2 . \overline{1} \quad$ Final-state showers

3.2 .2 Initial-state showers

Matrix-element corrections

$13.3 . \quad$ Heavy flavour production and decay

13.4. Gauge and Higgs boson decays

13.5. Supersymmetry

B. $\overline{5} . \overline{1}$ Data input

13. 5.2 Processes

1.5.5. Related changes

13.64 Hadronization

3. $3 . \overline{1} \overline{1} \quad$ Cluster model

3. 6.2 Underlying soft event

3. 3 Minimum bias processes

13.7. Spacetime structure

$3 . \overline{7} \quad$ Particle decays

3.7.2. Parton showers

Hadronization

3. Colour rearrangement

B- $\bar{B}$ mixing

i4. Processes

'T.'i' Beams

A.1.1.'Parton distributions

14.2 Summary of subprocesses

A. 1 Treatment of quark masses

$\overline{4} .2$ Couplings

14 Lepton-antilepton Standard Model processes

'A.3. I' IPROC $=100-127$ : hadron production

IPROC=150-250: lepton and electroweak boson production

APROC=300-499: Higgs boson production 
1.

IPROC $=600-656$ : four jet production

14.4epton-antilepton supersymmetric processes (MSSM)

IPROC=700-799: gaugino, slepton and/or squark production

14.5. Other lepton-antilepton non-Standard-Model processes

A.5. IPROC=800-899: R-parity violating SUSY processes

14.61 Hadron-hadron Standard Model processes

A. 6 . IPROC $=1300-1499:$ Drell-Yan processes

IPROC=1500: QCD $2 \rightarrow 2$ processes

IPROC = 1600-1699: Higgs boson production by parton fusion

IPROC=1700-1706: heavy quark production

APROC=1800: QCD direct photon plus jet production

A. $6 . \overline{6}$ IPROC=1900-1999: Higgs boson production by weak boson fusion

IA.6. IPROC $=2000-2008$ : single top production

IPROC $=2100-2170$ : electroweak boson plus jet production

IPROC=2200: direct photon pair production

19. 10 IPROC=2300-2399: Higgs boson plus jet production

1. IPROC $=2400-2450$ : colour singlet exchange

A

1. 6.13, IPROC $=2600-2799$ : Higgs plus weak boson production

1.7. Hadron-hadron supersymmetric processes (MSSM)

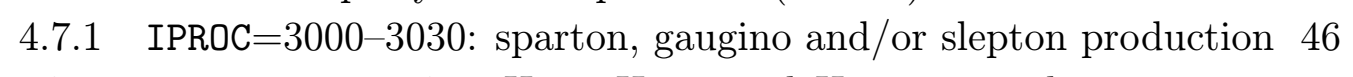

IA. IPROC=3310-3375: Higgs-Higgs and Higgs-gauge boson pair production

IT. $\overline{4}$ IPROC $=3410-3450$ : Higgs boson plus heavy quark production

APROC=3610-3630: neutral Higgs production by parton fusion

'4.

I. 7 IPROC $=4000-4199:$ R-parity violating SUSY processes

IPROC $=4200-4299$ : graviton resonance production

14. Photon-hadron and photon-photon processes

'4. $9 . \overline{1} \quad$ IPROC $=5000-5520$ : pointlike photon-hadron processes

IPROC=6000-6010: pointlike photon-photon processes

:4.10" Baryon number violating processes

1.10 IPROC=7000-7999: generated by the HERBVI package

4.

i. 1 IPROC=8000: Minimum bias soft hadron-hadron event

4.12 Deep inelastic lepton scattering

I.12.1; IPROC=9000-9006: neutral current

作

运 


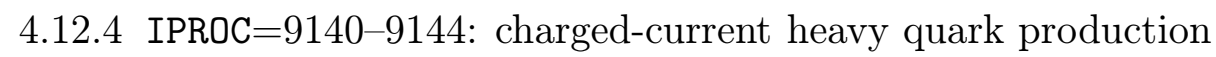

'A. $12 .{ }_{1}^{\prime}$ IPROC=9500-9599: Higgs boson production by weak boson fusion

'T. 13 Including new subprocesses

5. Parameters

6. Control switches, constants and options

it. Particle data

8. Structure and output

18.11 Main program

18.2 Form factor file

Event data

$8.3 .1, \quad$ Status codes

8.3 Event weights

18.4. Error conditions

Sample output

i $\overline{8} \cdot \overline{-} \cdot \overline{1}, \quad$ Guide to sample output

18.6 Subroutine descriptions

\section{Introduction}

HERWIG is a general-purpose event generator for high-energy processes, with particular emphasis on the detailed simulation of QCD parton showers. The program provides a full simulation of hard lepton-lepton, lepton-hadron and hadron-hadron scattering and soft hadron-hadron collisions in a single package, and has the following special features:

- Initial- and final-state QCD jet evolution with soft gluon interference taken into account via angular ordering;

- Colour coherence of (initial and final) partons in all hard subprocesses, including the production and decay of heavy quarks and supersymmetric particles;

- Azimuthal correlations within and between jets due to gluon interference and polarization;

- A cluster model for jet hadronization based on non-perturbative gluon splitting, and a similar cluster model for soft and underlying hadronic events; 
- A space-time picture of event development, from parton showers to hadronic decays, with an optional colour rearrangement model based on space-time structure.

Several of these features were already present in HERWIG version 5.1 and were described accordingly in some detail in ref. [ii]. This was in turn based on the work of refs. [2]-[i] corporated into HERWIG since 1992. The most important of these are the matching of

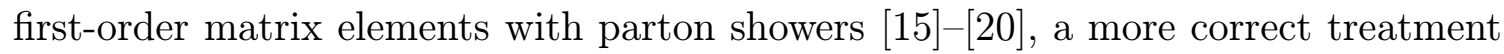

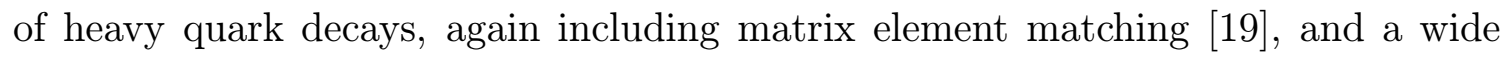
range of new hard processes. In particular HERWIG now includes the production and decay of supersymmetric particles, with or without the assumption of R-parity conservation [201].

The precise HERWIG version described here is 6.200 , which we shall generally refer to as "version 6 " in the following.

Let us recall briefly the main features of a generic hard, i.e. high momentum transfer, process of the type simulated by HERWIG. It can be divided notionally into four components, corresponding roughly to increasing scales of distance and time:

1. Elementary hard subprocess. A pair of incoming beam particles or their constituents interact to produce one or more primary outgoing fundamental objects. This can be computed exactly to lowest order in perturbation theory. The hard momentum transfer scale $Q$ together with the colour flow of the subprocess set the boundary conditions for the initial- and final-state parton showers, if there are any.

2. Initial- and final-state parton showers. A parton constituent of an incident beam hadron with low spacelike virtuality can radiate timelike partons. In the process it decreases its energy to a fraction $x$ of that of the beam and increases its spacelike virtuality, which is bounded in absolute value by the scale $Q$ of the hard subprocess. This initial-state emission process leads to the evolution of the structure function $F(x, Q)$ of the incident hadron. On a similar timescale, an outgoing parton with large timelike virtuality can generate a shower of partons with lower virtuality. The amount of emission depends on the upper limit on the virtuality of the initiating parton, which is again controlled by the momentum transfer scale $Q$ of the hard subprocess. Timelike partons from the initial-state emission may in turn initiate parton showering. The coherence of soft gluon emission from different parton showers is controlled by the colour flow of the subprocess. Within the showers, it is simulated by angular ordering of successive emissions.

3. Heavy object decays. Massive produced objects such as top quarks, electroweak gauge and Higgs bosons, and possibly non-Standard Model (e.g. SUSY) parti- 
cles, can decay on time-scales that may be shorter than or comparable to that of the QCD parton showers. Depending on their nature and the decay mode, they may also initiate parton showers before and/or after decaying.

4. Hadronization process. In order to construct a realistic simulation one needs to combine the partons into hadrons. This applies to the constituent partons of incoming hadronic beams as well as to the outgoing products of parton showering, which give rise to hadronic jets. This hadronization process takes place at a low momentum transfer scale, for which the strong coupling $\alpha_{\mathrm{S}}$ is large and perturbation theory is not applicable. In the absence of a firm theoretical understanding of non-perturbative processes, it must be described by a phenomenological model. The model adopted in HERWIG is intended to disrupt as little as possible the event structure established in the parton showering phase. Showering is terminated at a low scale, $Q_{0}<1 \mathrm{GeV}$, and the preconfinement property of perturbative QCD [2근, $\overline{2} \overline{2} \overline{3}]$ is exploited to form colour-neutral clusters [i] which decay into the observed hadrons. Initial-state partons are incorporated into the incoming hadron beams through a soft, nonperturbative "forced branching" phase of spacelike showering. The remnants of incoming hadron, i.e. those constituent partons which do not participate in the hard subprocess, undergo a soft "underlying event" interaction modelled on soft minimum bias hadron-hadron collisions.

After a brief, practical section on the use of HERWIG, in the following sections we discuss each of these components in turn, concentrating on the new features since version 5.1 .

\section{Using HERWIG}

The latest version of the program, together with all relevant information, is always available via the official HERWIG web page:

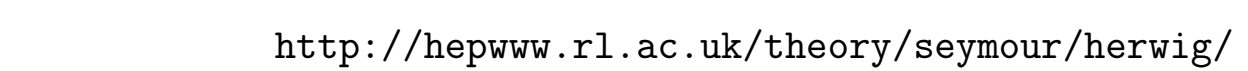

The program is written in Fortran and the user has to modify the main program HWIGPR to generate the type and number of events required. See section 8.1 . for a sample main program. The program operates by setting up parameters in common blocks and then calling a sequence of subroutines to generate an event. Parameters not set explicitly in the block data HWUDAT or in HWIGPR are set to default values in the main initialisation routine HWIGIN. Output data are delivered in the LEP standard

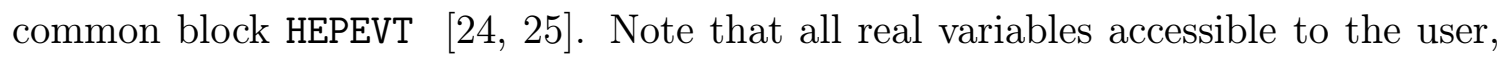
including those in HEPEVT, are of type DOUBLE PRECISION. 
To generate events the user must first set up the beam particle names PART1, PART2 (type CHARACTER*8) and momenta PBEAM1, PBEAM2 (in $\mathrm{GeV} / c$ ), a process code IPROC and the number of events required MAXEV.

See section '言for beams and processes available.

All analysis of generated events (histogramming, etc.) should be performed by the user-provided routines HWABEG (to initialise the run), HWANAL (to analyse an event) and HWAEND (to terminate the run).

A detailed event summary is printed out for the first MAXPR events (default MAXPR $=1$ ). Setting IPRINT $=2$ lists the particle identity codes, properties and decay schemes used in the program.

The programming language is standard Fortran 77 as far as possible. However, the following may require modification for running on some computers

- Most common blocks are inserted by INCLUDE 'HERWIG62. INC' statements the file HERWIG62. INC is part of the standard program package.

- Many common blocks are initialized by BLOCK DATA HWUDAT. Although BLOCK DATA is standard Fortran 77, it can cause linkage problems for some systems.

- Variables of type DOUBLE COMPLEX are used, which may be called COMPLEX*16 on some systems.

\section{Physics simulated by HERWIG}

\subsection{Elementary hard subprocess}

In HERWIG version 6 there is a fairly large library of QCD, electroweak and supersymmetric elementary subprocesses. These are listed and discussed in section 垈.

The hard subprocess plays an important rôle in defining the phase space of any associated initial- and final-state parton showers. As discussed in ref. [1. ences therein, the parton showers are branching processes in which the branchings are ordered in angle from a maximum to a minimum value determined by the cutoff $Q_{0}$. The maximum value is determined by the elementary subprocess and is due

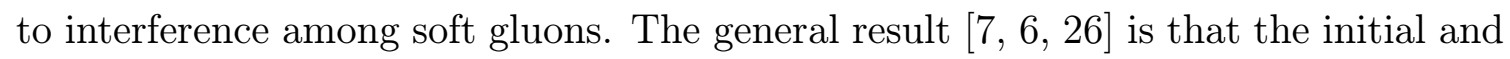
final branchings are approximately confined within cones around the incoming and outgoing partons from the elementary subprocess. For the branching of parton $i$, the aperture of the cone is defined by the direction of the other parton $j$ that is colour-connected to $i$. The relation between soft gluon interference and the colour connection structure of the elementary subprocess leads to many detectable effects in hadronic final states. For recent examples, see e.g. refs. [2] $27,1587$.

For a general process there are various contributions with different colour connections. The HERWIG library of elementary subprocesses includes the separate colour 
connection contributions. In general there is some ambiguity in the separation of contributions which are suppressed by inverse powers of the number of colours $N_{c}$. In earlier versions of HERWIG, these sub-leading terms were divided amongst the various colour connection contributions according to the recipe in ref. [i]i]. In version 6 the following improved prescription [29] has been followed, for both the QCD and SUSY QCD subprocesses. The matrix element squared $\left|\mathcal{M}_{i}\right|^{2}$ for each colour connection is computed in the limit $N_{c} \rightarrow \infty$ and the corresponding contribution is defined as

$$
\frac{\left|\mathcal{M}_{i}\right|^{2}}{\sum_{j}\left|\mathcal{M}_{j}\right|^{2}}|\mathcal{M}|^{2},
$$

where $|\mathcal{M}|^{2}$ represents the sum over all colour connections including terms sub-leading in $N_{c}$. This ensures that each colour connection contribution is positive-definite and has the correct pole structure, while the sum of contributions yields the exact (Bornlevel) cross section.

Parton emission into phase space regions which are outside the above-mentioned angular cones, called in the following "dead zones", do not contribute to leading order and often not even to next-to-leading order. However, for a more complete description of the event it is also necessary to take into account emission into these "dead zones", which may be done using exact fixed-order matrix elements (see the following sections).

Another important function of the elementary subprocess is to set up the polarizations of any electroweak bosons or gluon jets that may be involved. These polarizations give rise to angular asymmetries and correlations in boson decays and jet fragmentation. They are included in HERWIG for many of the subprocesses provided,

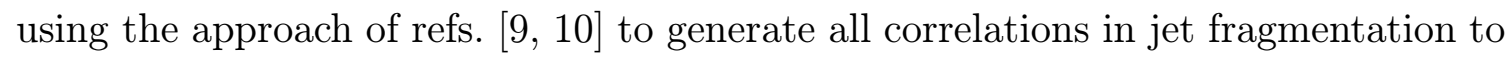
leading-logarithmic accuracy.

\subsection{Parton showers}

\subsubsection{Final-state showers}

Final-state parton showering in HERWIG is generated by a coherent branching algorithm with the following properties:

1. The energy fractions are distributed according to the leading-order DokshitzerGribov-Lipatov-Altarelli-Parisi (DGLAP) splitting functions.

2. The full available phase space is restricted to an angular-ordered region. Such a restriction is the result of interference and correctly takes leading infrared singularities into account. At each branching, the angle between the two emitted partons is smaller than that of the previous branching.

3. The emission angles are distributed according to the Sudakov form factors, which sum the virtual corrections and unresolved real emissions. The Sudakov 
form factor normalizes the branching distributions to give the probabilistic interpretation needed for a Monte Carlo simulation. This fact is a consequence of unitarity and of the infrared finiteness of inclusive quantities.

4. The azimuthal angular distribution in each branching is determined by two effects:

(a) for a soft emitted gluon the azimuth is distributed according to the eikonal dipole distribution [iiㅁ;

(b) for non-soft emission one finds azimuthal correlations due to spin effects.

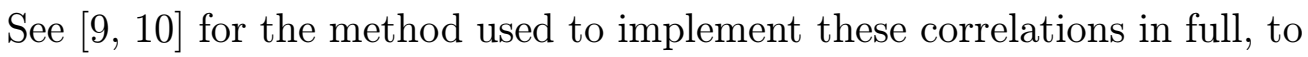
leading collinear logarithmic accuracy, in HERWIG.

5. In each branching the scale of $\alpha_{\mathrm{S}}$ is the relative transverse momentum of the two daughter partons.

6. In the case of heavy flavour production the mass of the quark modifies the angular-ordered phase space. The most important effect is that the soft radiation in the direction of the heavy quark is depleted. One finds that emission within an angle of order $M / E$ is suppressed, $M$ and $E$ being the mass and energy of the heavy quark: this is known as the "dead cone" $[\overline{3} \overline{0} \overline{0}$.

Specifically, the HERWIG parton shower evolution is done in terms of the parton energy fraction $z$ and an angular variable $\xi$. In the parton splitting $i \rightarrow j k, z_{j}=$ $E_{j} / E_{i}$ and $\xi_{j k}=\left(p_{j} \cdot p_{k}\right) /\left(E_{j} E_{k}\right)$. Thus $\xi_{j k} \simeq \frac{1}{2} \theta_{j k}^{2}$ for massless partons at small angles. The values of $z$ are chosen according to the DGLAP splitting functions and the distribution of $\xi$ values is determined by the Sudakov form factors. Angular ordering implies that each $\xi$ value must be smaller than the $\xi$ value for the previous branching of the parent parton.

The parton showers are terminated as follows. For partons there is a cutoff of the form $Q_{i}=m_{i}+Q_{0}$, where $m_{i}(i=1, \ldots, 6$ for $d, u, s, c, b, t)$ is set by the relevant mass parameter RMASS $(i)$ and $Q_{0}$ is set by the quark and gluon virtuality cutoff parameters VQCUT and VGCUT (see section $\overline{\underline{1}}$ ). Showering from any parton stops when a value of $\xi$ below $Q_{i}^{2} / E_{i}^{2}$ is selected for the next branching. For heavy quarks, the condition $\xi>Q_{i}^{2} / E_{i}^{2}$ corresponds to the "dead cone" mentioned above. At this point the parton is put on mass-shell or given a small non-zero effective mass in the case of gluons. ${ }^{1}$ Working backwards from these on-shell partons, one can now construct the virtual masses of all the internal lines of the shower and the overall jet mass, from the energies and opening angles of the branchings. Finally one can assign the azimuthal angles of the branchings, including EPR-type correlations (from Einstein-

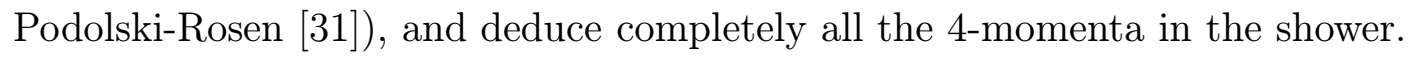

\footnotetext{
${ }^{1}$ The quark mass parameters should also be thought of as effective or constituent masses rather than current quark masses.
} 
Next the parton showers are used to replace the (on mass-shell) partons that were generated in the original hard process. This is done in such a way that the jet 3-momenta have the same directions as the original partons in the c.m. frame of the hard process, but they are boosted to conserve 4-momentum taking into account their extra masses.

The main improvements in the final-state emission algorithm of HERWIG version 6 , relative to version 5.1 , are as follows.

The Sudakov form factors can be calculated using the one-loop or two-loop $\alpha_{\mathrm{S}}$, according to the variable SUDORD (default $=1$ ). The parton showering still incorporates the two-loop $\alpha_{\mathrm{S}}$ in either case but if SUDORD $=1$ this is done using a veto algorithm, whereas if SUDORD $=2$ no vetoes are used in the final-state evolution. The usefulness of this option is discussed briefly in section 's. 1.21 .

Matrix element corrections have been introduced into final-state parton showers

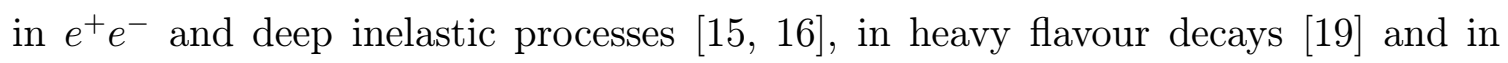

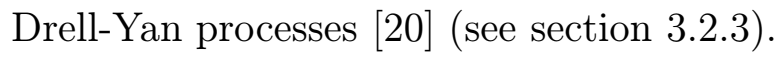

In HERWIG, the angular-ordering constraint, which is derived for soft gluon emission, is applied to all parton shower vertices, including $g \rightarrow q \bar{q}$. In versions before 6.1 , this resulted in a severe suppression (an absence in fact) of configurations in which the gluon energy is very unevenly shared between the quarks. For light quarks this is irrelevant, because in this region one is dominated by gluon emissions, which are correctly treated. However, for heavy quarks this energy sharing (or equivalently the quarks' angular distribution in their rest frame) is a directly measurable quantity and was badly described. Related to this was an inconsistency in the calculation of the Sudakov form factor for $g \rightarrow q \bar{q}$. This was calculated using the entire allowed kinematic range (with massless kinematics) for the energy fraction, $0 \leq z \leq 1$, while the $z$ distribution generated was actually confined to the angular-ordered region, $z, 1-z \geq m / E \sqrt{\xi}$.

In version 6 , these defects are corrected as follows. We generate the $E, \xi$ and $z$ values for the shower as before. We then apply an a posteriori adjustment to the kinematics of the $g \rightarrow q \bar{q}$ vertex during the kinematic reconstruction. At this stage, the masses of the $q$ and $\bar{q}$ showers are known. We can therefore guarantee to stay within the kinematically allowed region. In fact, the adjustment we perform is purely of the angular distribution of the $q$ and $\bar{q}$ showers in the $g$ rest frame, preserving all the masses and the gluon four-momentum. Therefore we do not disturb the kinematics of the rest of the shower at all.

Although this cures the inconsistency above, it actually introduces a new one: the upper limit for subsequent emission is calculated from the generated $E, \xi$ and $x$ values, rather than from the finally-used kinematics. This correlation is of NNLL importance, so we can formally neglect it. It would be manifested as an incorrect correlation between the masses and directions of the produced $q$ and $\bar{q}$ jets. This is, in principle, physically measurable, but it seems less important than getting the 
angular distribution itself right. In fact the solution we propose maps the old angular distribution smoothly onto the new, so the sign of the correlation will still be preserved, even if the magnitude is wrong. Even with this modification, the HERWIG kinematic reconstruction can only cope with particles that are emitted into the forward hemisphere in the showering frame. Thus one cannot populate the whole of kinematically-allowed phase space. Nevertheless, we find that this is usually a rather weak condition and that most of phase space is actually populated.

Using this procedure, we find that the predicted angular distribution for secondary $b$ quarks at LEP energies is well-behaved, i.e. it looks reasonably similar to the leading-order result $\left(1+\cos ^{2} \theta^{*}\right)$, and has relatively small hadronization corrections.

Real photon emission is included in timelike parton showering. The infrared photon cutoff is VPCUT, which defaults to $0.4 \mathrm{GeV}$. Agreement with LEP data is satisfactory if showering is used together with the matrix element correction to produce photons in the back-to-back region. The results are insensitive to VPCUT variations in the range $0.1-1.0 \mathrm{GeV}$. Setting VPCUT greater than the total c.m. energy switches off such emission. As an expedient way of improving the efficiency of photon final-state radiation studies, the electromagnetic coupling ALPHEM can be multiplied by a factor ALPFAC (default $=1$ ) for all quark-photon vertices in jets, and in the 'dead zone' in $e^{+} e^{-}$. Results at small photon-jet separation become sensitive to ALPFAC above about 5 .

\subsubsection{Initial-state showers}

The theoretical analysis of initial-state showering is more complex than the finalstate case. The most relevant parameters of the hard subprocess are the hard scale $Q$ and the energy fraction $x$ of the incoming parton after the emission of initial state radiation. For lepton-hadron processes $x$ corresponds to the Bjorken variable, while for hadron-hadron processes $x$ is related to $Q^{2} / s$ where $s$ is the c.m. energy squared.

The main result is that for any value of $x$, even for $x$ small [i]2], the initial-state emission process factorizes and can be described as a coherent branching process suitable for Monte Carlo simulations. The properties which characterize this process include all those discussed above for the final-state emission. However, in the initialstate case the angular-ordering restriction on the phase space applies to the angles $\theta_{i}$ between the directions of the incoming hadron and the emitted partons $i$.

For large $x$, the coherent branching algorithm sums correctly [1] 3 i] not only the leading but also the next-to-leading contributions. This accuracy allows us to identify the relation between the QCD scale used in the Monte Carlo program and the fundamental parameter $\Lambda_{\overline{\mathrm{MS}}}$. This is achieved by using the one-loop Altarelli-Parisi splitting functions and the two-loop expression for $\alpha_{\mathrm{S}}$ with the following universal relation between the scale parameter $\Lambda_{\text {phys }}$ [i $\overline{1} \overline{3}$, used in the simulation and $\Lambda_{\overline{\mathrm{MS}}}$ (here, 
$N_{f}$ is the number of flavours)

$$
\Lambda_{\text {phys }}=\exp \left(\frac{67-3 \pi^{2}-10 N_{f} / 3}{2\left(33-2 N_{f}\right)}\right) \Lambda_{\overline{\mathrm{MS}}} \simeq 1.569 \Lambda_{\overline{\mathrm{MS}}} \quad \text { for } N_{f}=5 .
$$

Therefore a Monte Carlo simulation with next-to-leading accuracy can be used to determine $\Lambda_{\overline{\mathrm{MS}}}$ from semi-inclusive data at large momentum fractions. ${ }^{2}$

In the case of small values of $x$, the initial state branching process has additional properties, which are not yet included fully in HERWIG. This was discussed in ref. [iㅔ] and the situation remains unchanged since version 5.1.

The initial-state branching algorithm in HERWIG is of the backward evolution type. It proceeds from the elementary subprocess, at a hard scale set by colour coherence (see section ' cutoff parameter QSPAC. At this point there is a forced non-perturbative stage of branching which ensures that the emitting parton fits smoothly with the valence parton distributions of the incoming hadron.

Matrix-element corrections have been introduced into initial-state parton showers

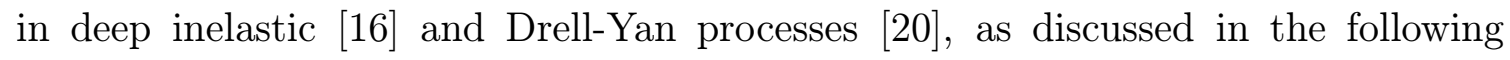
subsection.

To avoid double-counting of hard parton emission, all radiation at transverse momenta greater than the hard process scale EMSCA is vetoed. In the case of initialstate radiation, this affects all events, while for final-state radiation it only affects those events in which the two jets have a rapidity difference of more than about 3.4.

In the backward evolution of initial-state radiation for photons the anomalous branching $q \bar{q} \leftarrow \gamma$ is included. Variables ANOMSC(1,IBEAM) and ANOMSC(2,IBEAM) record the evolution scale and transverse momentum, respectively, at which an anomalous splitting was generated in the backward evolution of beam IBEAM. If zero, then no such splitting was generated.

The treatment of forced branching of gluons and sea (anti-)quarks in backward evolution has been improved, by allowing it to occur at a random scale between the spacelike cutoff QSPAC and the infrared cutoff, instead of exactly at QSPAC as before. A new option ISPAC $=2$ allows the freezing of structure functions at the scale QSPAC, while evolution continues to the infrared cutoff. The default, ISPAC $=0$ is equivalent to previous versions, in which perturbative evolution stops at QSPAC.

The width of the (gaussian) intrinsic transverse momentum distribution of valence partons in incoming hadrons at scale QSPAC is set by the parameter PTRMS (default value zero). The intrinsic transverse momentum is chosen before the initial state cascade is performed and is held fixed even if the generated cascade is rejected. This is done to avoid correlation between the amount of perturbative and non-perturbative transverse momentum generated.

\footnotetext{
${ }^{2}$ This applies also to final-state emission, i.e. to jet fragmentation at large values of the jet momentum fraction.
} 
It is possible to completely switch off initial-state emission, by setting NOSPAC $=$ .TRUE., in which case only the forced splitting of non-valence partons is generated.

\subsubsection{Matrix-element corrections}

One of the new features of HERWIG 6 is the matching of first-order matrix elements with parton showers.

The HERWIG parton showers are performed in the soft or collinear approximation and emission is allowed only in regions of the phase space satisfying the condition $\xi<$ 1 or $\xi<z^{2}$, for the final- (timelike branching) and initial-state (spacelike branching) radiation respectively, where $\xi$ and $z$ are the showering variables defined above.

The emission is entirely suppressed inside the so-called dead zones $(\xi>1$ or $\xi>z^{2}$ ), corresponding to hard and/or large-angle parton radiation. According to the exact matrix elements, the radiation in the dead zones is suppressed, since it is not soft or collinear logarithmically enhanced, but it is not completely absent as happens in the HERWIG standard shower algorithm. The HERWIG parton cascades need to be supplemented by matrix-element corrections for a full description of the physical phase space.

The method of matrix-element corrections to the HERWIG parton showers is dis-

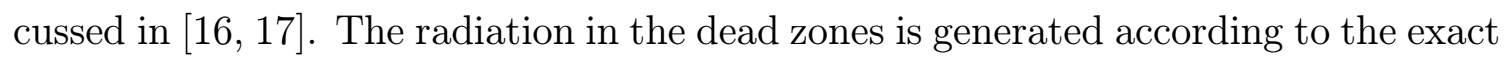
first-order matrix element ('hard correction'); the shower in the already-populated region of the phase space is corrected by the use of the exact $\mathcal{O}\left(\alpha_{S}\right)$ amplitude any time an emission is capable of being the 'hardest so far' ('soft correction'3).

By 'hardest-so-far', we mean the radiation of a parton whose transverse momentum relative to the splitting one is larger than all those previously emitted. This is not always the first emission, as angular ordering does not necessarily imply ordering in transverse momentum. As shown in [1] $\left[\begin{array}{l}6] \\ \text { ] }\end{array}\right.$, if we corrected only the first emission, we would have problems in the implementation of the Sudakov form factor whenever a subsequent harder emission occurs, as we would find that the probability of hard radiation would depend on the infrared cutoff, which is clearly unphysical. Using the $\mathcal{O}\left(\alpha_{S}\right)$ result for the hardest-so-far emission in the already-filled phase space as well as in the dead zone allows one to have matching over the boundary of the dead zone itself.

Since the fraction of events which receive a hard correction is typically small, we neglect multiple hard emissions in the dead zones and rely on the first-order result plus showering in those regions.

Our method is quite different from the one used to implement matrix-element cor-

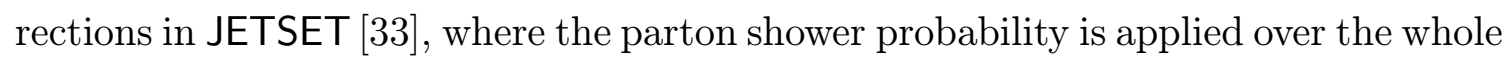
phase space and the first-order amplitude is used only to correct the first emission.

\footnotetext{
${ }^{3}$ We point out that in the expression 'soft correction', 'soft' refers to the phase space where such corrections are applied and not to the amplitude, since we still use the 'hard' exact matrix element for the soft correction as well.
} 
Following these general prescriptions, matrix-element corrections have been implemented in some $e^{+} e^{-}$processes [1]

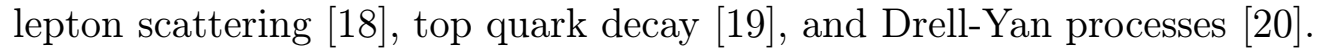

The variables HARDME (default $=$. TRUE. $)$ and SOFTME (default $=$. TRUE.) allow respectively the application of hard and soft matrix-element corrections to the HERWIG parton cascades.

\subsection{Heavy flavour production and decay}

Heavy quark decays are treated as secondary hard subprocesses. Top quarks and any hypothetical heavier quarks always decay before hadronization. Heavy-flavoured hadrons are split into collinear heavy quark and spectator and the former decays independently. After decay, parton showers may be generated from coloured decay products, in the usual way. See ref. [i] for details of the treatment of colour coherence in these showers.

In HERWIG version 6 matrix-element corrections to the simulation of top quark decays are available. The routine HWBTOP implements the hard corrections; HWBRAN has been modified to implement the soft corrections. Since the dead zone includes part of the soft singularity, a cutoff is required: only gluons with energy above GCUTME (default value $2 \mathrm{GeV}$ ) in the top rest frame are corrected. Physical quantities are not strongly dependent on GCUTME in the range 1 to $5 \mathrm{GeV}$, after the typical experimental cuts are applied. For more details see ref. [i 19 .

The structure of the program has been altered so that the secondary hard subprocess and subsequent fragmentation associated with each partonic heavy hadron decay appear separately in the event record. Thus top quark decays are treated individually as are any subsequent bottom hadron partonic decays. Note that the statement CALL HWDHOB, which deals with the decays of all heavy objects (including SUSY particles), must appear in the main program between the calls to HWBGEN and HWCFOR, in order to carry out any decays before hadronization.

The partonic decay fractions of heavy quarks are specified in the decay tables like the decay modes of other particles. This permits different decays to be given to individual heavy hadrons. Changes to the decay table entries can be made on an event by event basis if desired. Partonic decays of charm hadrons and quarkonium states are also now supported. The order of the products in a partonic decay mode is significant. For example, if the decay is $Q \rightarrow W+q \rightarrow\left(f+\bar{f}^{\prime}\right)+q$ occurring inside a $Q \bar{s}$ hadron, the required orderings are:

$$
\begin{aligned}
Q+\bar{s} & \rightarrow\left(f+\bar{f}^{\prime}\right)+(q+\bar{s}) \\
& \text { or }\left(q+\bar{f}^{\prime}\right)+(f+\bar{s}) \quad \text { ('colour rearranged') } .
\end{aligned}
$$

In both cases the $(V-A)^{2}$ matrix element-squared is proportional to $\left(p_{Q} \cdot p_{2}\right)\left(p_{1} \cdot p_{3}\right)$, where $p_{1}$ etc. correspond to the ordering given. Decays of heavy-flavoured hadrons 
to exclusive non-partonic final states are also supported. No check is made against double counting from partonic modes. However this is not expected to be a major problem for the semi-leptonic and two-body hadronic modes supplied.

The default masses of the $c$ and $b$ quarks have been lowered to 1.55 and 4.95 respectively: this corresponds to the mass of the lightest meson minus the $u$ or $d$ quark mass. This increases the number of heavy mesons, and hence total multiplicities, and slightly softens their momentum spectrum. The rate of photoproduced charm states increases and B- $\pi$ momentum correlations become smoother. The default top quark mass is $174.3 \mathrm{GeV} / c^{2}$. The same value is used in the production and decay matrix elements and for all kinematics. Note that higher-order corrections are not fully included, and so the HERWIG top mass does not necessarily correspond to that defined in any particular rigorous scheme (e.g. the pole mass or the $\overline{\mathrm{MS}}$ running mass). However, since it is probably the decay kinematics that are most sensitive to this parameter, it should be close to the pole mass. See subsection the treatment of quark masses in various processes.

\subsection{Gauge and Higgs boson decays}

The total decay widths of the electroweak gauge bosons $V=W, Z$ are specified by the input parameters GAMW and GAMZ. Their branching fractions to various final states are computed automatically from the other SM input parameters. Which decays actually occur is controlled as follows. The variable $\operatorname{MODBOS}(i)$ controls the decay of the $i$ th gauge boson per event (table $\left.i_{-1}^{1}\right)$.

All entries of MODBOS default to 0 . Bosons which are produced in pairs (i.e.

\begin{tabular}{|c|c|c|}
\hline $\operatorname{MODBOS}(i)$ & $W^{ \pm}$Decay & $Z^{0}$ Decay \\
\hline 0 & all & all \\
1 & $q \bar{q}$ & $q \bar{q}$ \\
2 & $e \nu$ & $e^{+} e^{-}$ \\
3 & $\mu \nu$ & $\mu^{+} \mu^{-}$ \\
4 & $\tau \nu$ & $\tau^{+} \tau^{-}$ \\
5 & $e \nu+\mu \nu$ & $e^{+} e^{-}+\mu^{+} \mu^{-}$ \\
6 & all & $\nu \bar{\nu}$ \\
7 & all & $b \bar{b}$ \\
$>7$ & all & all \\
\hline
\end{tabular}

Table 1: $W, Z$ decays. from $V V$ pair production, or Higgs decay) are symmetrized in $\operatorname{MODBOS}(i)$ and $\operatorname{MODBOS}(i+1)$. For processes which directly produce gauge bosons, the event weight includes the branching fraction to the requested decay, but this is only true for Higgs production if decay to $W^{+} W^{-} / Z^{0} Z^{0}$ is forced (IPROC $=310,311$ but not 399, etc.). Users can thus force $Z \rightarrow b \bar{b}$ decays, with $\operatorname{MODBOS}(i)=7$. For example, $\operatorname{IPROC}=250, \operatorname{MODBOS}(1)=7, \operatorname{MODBOS}(2)=0$ gives $Z^{0} Z^{0}$ production with one $Z^{0}$ decaying to $b \bar{b}$.

The spin correlations in the decays are handled in one of two ways:

1. The diagonal members of the spin density matrix are stored in $\operatorname{RHOHEP}(i, \operatorname{IHEP})$, where $i=1,2,3$ for helicity $=i-2$ in the centre-of-mass frame of their produc- 
tion, for processes where this matrix is diagonal (i.e. there is no interference between spin states).

2. The correlations in the decay are handled directly by the production routine where (1) is not possible.

The processing of the parton showers in hadronic $W$ and $Z$ decays is handled in the rest frame of the vector boson if WZRFR is . TRUE. (the default), otherwise in the lab frame. In the latter case, which was the default in earlier versions, the initial cone angles of the showers depend on the velocity of the boson, which leads to a slight Lorentz non-invariance of decay distributions.

The total decay width of the SM Higgs boson is computed from its input mass RMASS (201) and stored as GAMH. Its decay branching fractions are also computed and stored in BRHIG(I): I $=1-6$ for $d \bar{d}, \ldots, t \bar{t} ; \mathrm{I}=7-9$ for $e^{+} e^{-}, \ldots, \tau^{+} \tau^{-} ; \mathrm{I}=10,11,12$ for $W^{+} W^{-}, Z^{0} Z^{0}, \gamma \gamma$. Non-SM Higgs bosons, on the other hand, such as those in supersymmetric models, have to have their widths and decay tables provided as input data (see section is.5. distinct identity code in HERWIG and is represented by the special symbol $H_{\mathrm{SM}}^{0}$.

There are two choices for the treatment of the SM Higgs width, both controlled by the variable IOPHIG:

$$
\mathrm{IOPHIG}=2 I+J
$$

where $I$ and $J$ are both zero or one. Whenever a Higgs boson is generated, its mass is chosen from a distribution that, for heavy SM Higgs bosons, can be rather broad. The choice of $I$ makes a significant difference to the physical meaning of the distribution generated: for $I=0$, the cross section corresponds to the tree level process containing an $s$-channel Breit-Wigner resonance for the Higgs boson with a running Higgs width. As discussed in [3- $\left.{ }^{3} \overline{4}\right]$, this neglects important contributions from interference with non-resonant diagrams and can violate unitarity at high energy, so

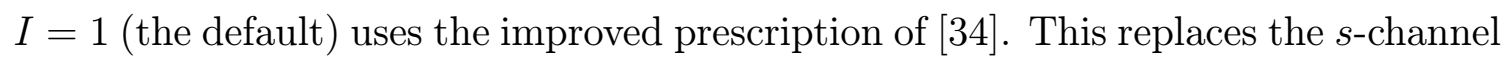
propagator by an effective propagator that sums the interference terms to all orders. This increases the cross section below resonance and decreases it above, causing an overall increase in cross section. More details can be found in [푸교.

The variable $J$ is a more technical parameter that does not affect the physical results, only the method by which they are generated: $J=1$ (the default) generates the mass according to a fixed-width Breit-Wigner resonance, while $J=0$ biases the distribution more towards higher masses. In either case, the appropriate jacobian factor is included in the event weight, so that the physical cross section is independent of $J$.

In all the above cases, the SM Higgs mass distribution is restricted to the range $\left[m_{\mathrm{H}}-\right.$ GAMMAX $\times \Gamma_{\mathrm{H}}, m_{\mathrm{H}}+$ GAMMAX $\left.\times \Gamma_{\mathrm{H}}\right]$. GAMMAX defaults to 10 , but in the nonperturbative region $m_{\mathrm{H}} \gtrsim 1 \mathrm{TeV}$ should be reduced to protect against poor weight 
distributions. These considerations do not affect the distribution noticeably for $m_{\mathrm{H}} \lesssim 500 \mathrm{GeV}$, and GAMMAX can safely be increased if necessary.

For a SUSY Higgs, the width is never large enough for unitarity to be violated and these issues are unimportant. In this case, the mass distribution is chosen according to a fixed-width Breit-Wigner resonance, like that of any other SUSY particle.

The SM Higgs decays that can occur are normally controlled by the process code IPROC, as in IPROC $=300+$ ID for example: ID $=1-6$ for quarks, $7-9$ for leptons, $10 / 11$ for $W^{+} W^{-} / Z^{0} Z^{0}$ pairs, and 12 for photons. In addition ID $=0$ gives quarks of all flavours, and $I D=99$ gives all decays. For each process, the average event weight is the cross section in nb times the branching fraction to the requested decay. The branching ratios to quarks use the next-to-leading logarithm corrections, those to $W^{+} W^{-} / Z^{0} Z^{0}$ pairs allow for one or both bosons being off mass-shell.

All Higgs vertices include an optional enhancement factor to account for non-SM and non-MSSM couplings. The amplitudes for all Higgs vertices are multiplied by the factor ENHANC (ID) where ID is the same as in IPROC $=300+$ ID except the $\gamma \gamma H$ 'vertex' which is calculated from ENHANC (6) and ENHANC (10) for the top and $W^{ \pm}$ loops. This allows the simulation of the production of any chargeless scalar Higgs-like particle. Note however that pseudoscalar and charged Higgs bosons, and processes involving more than one Higgs particle (e.g. the decay $H^{0} \rightarrow h^{0} Z$ ) are not included this way (see section $\left.{ }_{1}^{4} . \overline{7}_{1}\right)$.

The array ENHANC(ID) is initialised as usual in HWIGIN. Note, however, that it will be overwritten if MSSM Higgs production is required by IPROC. In that case, as mentioned earlier, the Higgs widths and decay modes are simply read from an input particle data file (see section $3 . \overline{3} \cdot \overline{1} \cdot \overline{1}$ ).

\subsection{Supersymmetry}

HERWIG now includes the production and decay of superparticles, as given by the

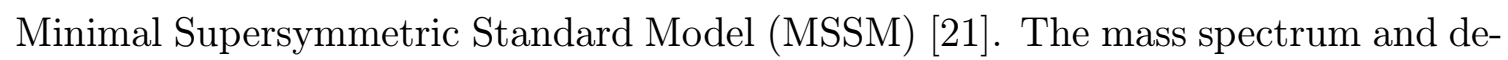
cay modes, being read from input files (see below), are completely general. The particle content, listed in the following table (table ${ }_{2}^{2}$ i) , includes the gravitino/goldstino. For sparticles that mix, the subscripts label the mass eigenstates in the ascending order of mass. The two Higgs Doublet Model (2HDM) Higgs sector, intrinsic to the MSSM, is also included. The three neutral Higgs bosons are denoted by $h^{0}, H^{0}$ and $A^{0}$.

The SUSY particle names in HERWIG are as shown in table $\overline{3 .}$. IDHW is the

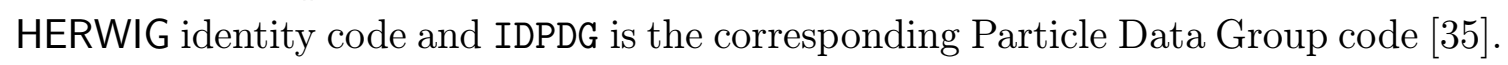

Antiparticles generally appear in sequence after the corresponding particles, e.g. antisquarks $\widetilde{d}_{L}^{*}-\widetilde{t_{1}^{*}}$ at IDHW $=407-412, \widetilde{d}_{R}^{*}-\widetilde{t}_{2}^{*}$ at $419-424$. They have 'BR' added to the name, e.g. 'SSDLBR ', or opposite charge, and negative PDG codes. A full list can be obtained using the print option IPRINT $=2$ (see section $\left.\underline{6}^{\prime} \bar{i}^{i}\right)$. 


\begin{tabular}{|c|c|c|c|c|c|}
\hline Particle & & Spin & Particle & & Spin \\
\hline quark & $q$ & $1 / 2$ & squarks & $\widetilde{q}_{L, R}$ & 0 \\
\hline charged lepton & $\ell$ & $1 / 2$ & charged sleptons & $\tilde{\ell}_{L, R}$ & 0 \\
\hline neutrino & $\nu$ & $1 / 2$ & sneutrino & $\widetilde{\nu}$ & 0 \\
\hline gluon & $g$ & 1 & gluino & $\widetilde{g}$ & $1 / 2$ \\
\hline photon & $\gamma$ & 1 & photino & $\widetilde{\gamma}$ & $1 / 2$ \\
\hline neutral gauge boson & $Z^{0}$ & 1 & zino & $\widetilde{Z}$ & $1 / 2$ \\
\hline neutral Higgs bosons & $h^{0}, H^{0}, A^{0}$ & 0 & neutral Higgsinos & $\widetilde{H}_{1,2}^{0}$ & $1 / 2$ \\
\hline charged gauge boson & $W^{ \pm}$ & 1 & wino & $\widetilde{W}^{ \pm}$ & $1 / 2$ \\
\hline charged Higgs boson & $H^{ \pm}$ & 0 & charged Higgsino & $\widetilde{H}^{ \pm}$ & $1 / 2$ \\
\hline graviton & $G$ & 2 & gravitino & $\widetilde{G}$ & $3 / 2$ \\
\hline \multicolumn{6}{|c|}{$\begin{array}{c}\widetilde{W}^{ \pm}, \widetilde{H}^{ \pm} \text {mix to form } 2 \text { chargino mass eigenstates } \widetilde{\chi}_{1}^{ \pm}, \widetilde{\chi}_{2}^{ \pm} \\
\widetilde{\gamma}, \widetilde{Z}, \widetilde{H}_{1,2}^{0} \text { mix to form } 4 \text { neutralino mass eigenstates } \widetilde{\chi}_{1}^{0}, \widetilde{\chi}_{2}^{0}, \widetilde{\chi}_{3}^{0}, \widetilde{\chi}_{4}^{0} \\
\left.\widetilde{t}_{L}, \widetilde{t}_{R} \text { (and similarly } \widetilde{b}, \widetilde{\tau}\right) \text { mix to form the mass eigenstates } \widetilde{t}_{1}, \widetilde{t}_{2}\end{array}$} \\
\hline
\end{tabular}

Table 2: SUSY particle content.

Note that the HERWIG particle labelling of the lightest MSSM Higgs boson departs from the PDG recommendation: it is given PDG code 26, to avoid confusion with the SM Higgs boson (PDG code 25) in our implementation (specifically, in our use of the array ENHANC for the MSSM processes: see the relevant Higgs sections for more details).

HERWIG does not contain any built-in models for SUSY scenarios beyond the MSSM, such as, Supergravity (SUGRA) or Gauge Mediated Symmetry Breaking (GMSB). In all cases the SUSY particle spectrum and decay tables must be provided just like those for any other particles. The subroutine HWISSP, if called, reads these from an input file. The production subprocesses are then generated by HWHESP, in lepton-antilepton collisions, HWHSSP, in hadron-hadron collisions, or by one of the $R_{\mathrm{p}}$ production routines. The decays of the sparticles produced, as well as any top quarks or Higgs bosons, are then performed by HWDHOB.

\subsubsection{Data input}

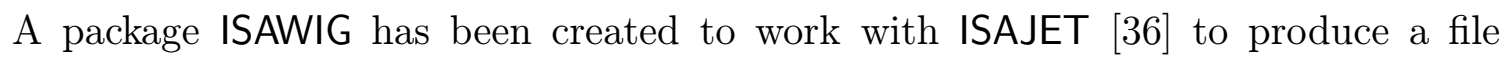
containing the SUSY particle masses, lifetimes and decay modes. This package takes the outputs of the ISAJET SUGRA or general MSSM programs and produces a data file in a format that can be read into HERWIG by the subroutine HWISSP. In principle the user can produce a similar file provided that the correct format is used, as explained below.

For the mixing terms of the MSSM lagrangian we follow the Haber-Kane [3] conventions, so that we differ from ISAJET on the sign for gaugino masses, the ordering and signs of the gaugino current eigenstates, the interchange of the rows 


\begin{tabular}{|c|c|c|c|c|c|c|c|}
\hline IDHW & & NAME & IDPDG & IDHW & & NAME & IDPDG \\
\hline 401 & $\widetilde{d}_{L}$ & 'SSDL ' & 1000001 & 413 & $\widetilde{d}_{R}$ & 'SSDR ' & 2000001 \\
\hline 402 & $\widetilde{u}_{L}$ & 'SSUL ' & 1000002 & 414 & $\widetilde{u}_{R}$ & 'SSUR ' & 2000002 \\
\hline 403 & $\widetilde{s}_{L}$ & 'SSSL ' & 1000003 & 415 & $\widetilde{s}_{R}$ & 'SSSR ' & 2000003 \\
\hline 404 & $\widetilde{c}_{L}$ & 'SSCL ' & 1000004 & 416 & $\widetilde{c}_{R}$ & 'SSCR ' & 2000004 \\
\hline 405 & $\widetilde{b}_{1}$ & 'SSB1 ' & 1000005 & 417 & $\widetilde{b}_{2}$ & 'SSB2 ' & 2000005 \\
\hline 406 & $\widetilde{t}_{1}$ & 'SST1 ' & 1000006 & 418 & $\widetilde{t}_{2}$ & 'SST2 ' & 2000006 \\
\hline 425 & $\widetilde{e}_{L}$ & 'SSEL- ' & 1000011 & 437 & $\widetilde{e}_{R}$ & 'SSER- ' & 2000011 \\
\hline 426 & $\widetilde{\nu}_{e}$ & 'SSNUEL ' & 1000012 & & & & \\
\hline 427 & $\tilde{\mu}_{L}$ & 'SSMUL- ' & 1000013 & 439 & $\widetilde{\mu}_{R}$ & 'SSMUR-' & 2000013 \\
\hline 428 & $\widetilde{\nu}_{\mu}$ & 'SSNUMUL ' & 1000014 & & & & \\
\hline 429 & $\tilde{\tau}_{1}$ & 'SSTAU1- ' & 1000015 & 441 & $\widetilde{\tau}_{2}$ & 'SSTAU2- ' & 2000015 \\
\hline 430 & $\widetilde{\nu}_{\tau}$ & 'SSNUTL ' & 1000016 & & & & \\
\hline 449 & $\widetilde{g}$ & 'GLUINO ' & 1000021 & 458 & $\widetilde{G}$ & 'GRAVTINO' & 1000039 \\
\hline 450 & $\widetilde{\chi}_{1}^{0}$ & 'NTLINO1 ' & 1000022 & 451 & $\tilde{\chi}_{2}^{0}$ & 'NTLINO2 ' & 1000023 \\
\hline 452 & $\widetilde{\chi}_{3}^{0}$ & 'NTLINO3 ' & 1000025 & 453 & $\tilde{\chi}_{4}^{0}$ & 'NTLINO4 ' & 1000035 \\
\hline 454 & $\tilde{\chi}_{1}^{+}$ & 'CHGINO1+' & 1000024 & 455 & $\tilde{\chi}_{2}^{+}$ & 'CHGINO2+' & 1000037 \\
\hline 204 & $h^{0}$ & 'HIGGSLO ' & 26 & 205 & $H^{0}$ & 'HIGGSHO ' & 35 \\
\hline 206 & $A^{0}$ & 'HIGGSAO ' & 36 & 207 & $H^{+}$ & 'HIGGS+ ' & 37 \\
\hline
\end{tabular}

Table 3: SUSY particle names.

and columns of the gaugino mixing matrices, and the sign of the neutral Higgs mixing angle $\alpha$.

In addition to the decay modes included in the ISAJET package ISAWIG allows for the possibility of violating R-parity and includes the calculation of all 2-body squark and slepton, and 3-body gaugino and gluino R-parity violating $\left(R_{\mathrm{p}}\right)$ decay modes.

It can happen that some of the SUSY particle decay modes generated by ISAJET are found to be kinematically forbidden in HERWIG, owing to the slightly different values assumed for the light quark masses. In this case a warning message is printed by HERWIG and these modes are deleted, the other branching ratios being rescaled accordingly. Such modes normally have negligible ISAJET branching ratios anyway, because of their tiny phase space.

The input file organisation expected by HWISSP is as follows. First the SUSY particle and top quark masses and lifetimes (in seconds) are given according to their HERWIG identity codes IDHW, for example:

65

$401927.3980 \quad 0.74510 \mathrm{E}-25$ 
That is,

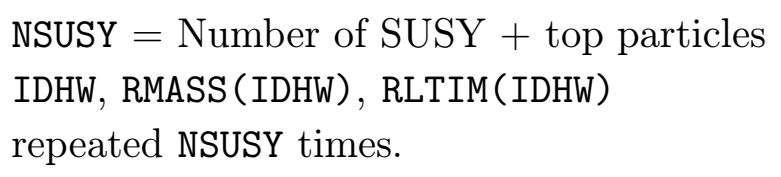

Next each particle's decay modes together with their branching ratios and matrix element codes are given as, for example:

$\begin{array}{lllccccc}6 & & & & & & & \\ 401 & 0.18842796 \mathrm{E}-01 & 0 & 450 & 1 & 0 & 0 & 0 \\ \quad: & : & : & : & : & : & : & : \\ 401 & 0.32755006 \mathrm{E}-02 & 0 & 457 & 2 & 0 & 0 & 0 \\ 6 & & & & & & & \\ 402 & 0.94147678 \mathrm{E}-02 & 0 & 450 & 2 & 0 & 0 & 0 \\ \ldots \text {. etc. }\end{array}$

That is,

Number of decay modes for a given particle IDK

IDK (IM), BRFRAC (IM), NME(IM), IDKPRD (1-5, IM)

repeated for each mode IM

all repeated for each particle (NSUSY times).

The order in which the decay products appear is important in order to obtain appropriate showering and hadronization. The correct orderings are indicated in the table below (table 'in').

A new matrix element code has been added for SUSY decays:

- $\mathrm{NME}=300$ for three-body $R_{\mathrm{p}}$ gaugino and gluino decays.

The indices $i, j, k$ in $R_{\mathrm{p}}$ gaugino/gluino decays refer to the ordering of the indices in the $R_{\mathrm{p}}$ couplings in the superpotential. The convention is as in ref. [3.

Next a number of parameters derived from the SUSY lagrangian must be given. These are: the ratio of Higgs VEVs, $\tan \beta$, and the scalar Higgs mixing angle, $\alpha$; the mixing parameters for the Higgses, gauginos and the sleptons; the trilinear couplings; and the Higgsino mass parameter $\mu$.

Finally the logical variable RPARTY must be set .FALSE. if R-parity is violated, and the $R_{\mathrm{p}}$ couplings must also be supplied; otherwise not.

Details of the FORMAT statements employed can be found by examining the subroutine HWISSP. 


\begin{tabular}{|c|c|c|c|c|c|}
\hline \multirow{2}{*}{$\begin{array}{l}\text { Decaying } \\
\text { Particle }\end{array}$} & \multirow{2}{*}{$\begin{array}{l}\text { No. of } \\
\text { products }\end{array}$} & \multirow[t]{2}{*}{ Type of mode } & \multicolumn{3}{|c|}{ Order of decay products } \\
\hline & & & 1 st & $2 \mathrm{nd}$ & $3 \mathrm{rd}$ \\
\hline \multirow[t]{2}{*}{ top } & 2 & two-body to Higgs & Higgs & bottom & \\
\hline & 3 & three-body via Higgs/W & \multicolumn{2}{|c|}{$\begin{array}{l}\text { quarks or leptons } \\
\text { from W/Higgs }\end{array}$} & bottom \\
\hline \multirow[t]{3}{*}{ gluinos } & \multirow[t]{2}{*}{2} & without gluon & \multicolumn{2}{|c|}{ any order } & \\
\hline & & with gluon & gluon & $\begin{array}{l}\text { colour } \\
\text { neutral }\end{array}$ & \\
\hline & 3 & R-parity conserved & $\begin{array}{c}\text { colour } \\
\text { neutral }\end{array}$ & \multicolumn{2}{|c|}{ quark or antiquark } \\
\hline \multirow[t]{2}{*}{$\begin{array}{c}\text { squark } \\
\text { or slepton }\end{array}$} & 2 & $\begin{array}{l}\text { gaugino/gluino } \\
\text { quark/lepton }\end{array}$ & $\begin{array}{l}\text { gaugino } \\
\text { gluino }\end{array}$ & $\begin{array}{l}\text { quark } \\
\text { lepton }\end{array}$ & \\
\hline & 3 & weak & sparticle & \multicolumn{2}{|c|}{$\begin{array}{c}\text { particles from } \\
\text { W decay }\end{array}$} \\
\hline \multirow[t]{2}{*}{ squarks } & \multirow[t]{2}{*}{2} & lepton number violated & quark & lepton & \\
\hline & & baryon number violated & quark & quark & \\
\hline sleptons & 2 & lepton number violated & \multicolumn{2}{|c|}{ quark or antiquark } & \\
\hline \multirow[t]{3}{*}{ Higgs } & \multirow[t]{2}{*}{2} & (s)quark-anti(s)quark & \multicolumn{2}{|c|}{ (s)quark or anti(s)quark } & \\
\hline & & (s)lepton-anti(s)lepton & \multicolumn{2}{|c|}{ (s)lepton or anti(s)lepton } & \\
\hline & 3 & all three-body & $\begin{array}{l}\text { colour } \\
\text { neutral }\end{array}$ & \multicolumn{2}{|c|}{$\begin{array}{l}\text { quark or antiquark } \\
\text { lepton or antilepton }\end{array}$} \\
\hline \multirow[t]{3}{*}{ gaugino } & 2 & squark-quark & \multicolumn{2}{|c|}{ quark or squark } & \\
\hline & & slepton-lepton & \multicolumn{2}{|c|}{ lepton or slepton } & \\
\hline & 3 & R-parity conserved & $\begin{array}{l}\text { colour } \\
\text { neutral }\end{array}$ & \multicolumn{2}{|c|}{$\begin{array}{l}\text { quark or antiquark } \\
\text { lepton or antilepton }\end{array}$} \\
\hline $\begin{array}{l}\text { gaugino } \\
\text { or gluino }\end{array}$ & 3 & R-parity violating & \multicolumn{3}{|c|}{$\begin{array}{l}\text { particles in the order } i, j, k \text { as } \\
\text { in the superpotential }\end{array}$} \\
\hline
\end{tabular}

Table 4: SUSY decay product ordering.

HWISSP reads the data from UNIT $=$ LRSUSY (default LRSUSY $=66$ ). If the data are stored in a fort.LRSUSY file on a UNIX system ${ }^{4}$ no further action is required, but if the data are to be read from a file named fname. dat then appropriate OPEN and CLOSE statements must be added by hand:

OPEN (UNIT=LRSUSY , FORM= ' FORMATTED ' , STATUS= 'UNKNOWN ' , FILE=' fname . dat' ' ) CALL HWISSP CLOSE (UNIT=LRSUSY)

A number of sets of SUSY parameter files, produced using ISAJET, for the standard LHC SUGRA and GMSB points are available from the HERWIG home page: http

\footnotetext{
${ }^{4}$ Or the equivalent, e.g. fortLRSUSY dat on a VAX system.
} 


\subsubsection{Processes}

The implementation of supersymmetric particle production processes in lepton-anti-

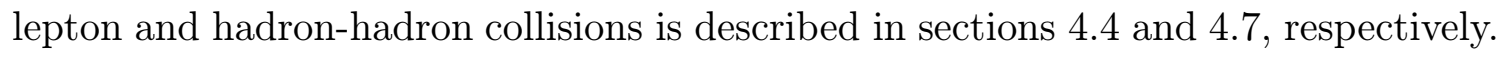
As in SM processes, we do not include any higher-order QCD corrections to the relevant matrix elements, even though these are now known in many cases. This is in order to avoid double counting of corrections generated approximately by parton showering. The upgrade toward the inclusion of MSSM Higgs processes in $e^{+} e^{-}$ collisions and of SUSY and MSSM Higgs processes in $e^{ \pm} p$ scattering is under way.

The procedure by which the MSSM matrix elements describing the elementary hard subprocesses are interfaced to the initial- and final-state parton showers is similar to that described in sections to. showers are generated from partons but not from spartons, whose short lifetimes should make this a reasonable approximation.

For the decay of SUSY particles we use for the moment only phase space weights rather than the exact decay matrix elements, apart from the case of three body $R_{\mathrm{p}}$ decays. However, where stated (e.g. in Higgs $\rightarrow W^{+} W^{-}$and $Z^{0} Z^{0}$ ) the spin information of the decay process is retained exactly. Breit-Wigner mass distributions have been implemented for all unstable SUSY particles, according to their widths as given in the input file. For a list of the most relevant decay modes of MSSM particles, see ref. [2]1.

One difference between the SM and MSSM implementations of the Higgs decay channels should be mentioned. Whereas for the SM Higgs boson all decay rates are calculated by HERWIG itself, in terms of the other (known) parameters of the SM, in the case of the MSSM scalars these are passed to the generator through the data files.

A more detailed description of the new SUSY reactions in HERWIG, along with the relevant formulae for the hard scattering processes involved, can be found in $\left[\overline{2} \bar{I}_{1}\right]$.

We include the possibility of violating R-parity both in the decays of the sparticles and in the initial hard subprocess. The $R_{\mathrm{p}}$ model we consider is specified by a spectrum which can be given in either the SUGRA, GMSB or a more general MSSM scenario, and a set of $R_{\mathrm{p}}$ couplings at the weak scale. We include only the tri-linear couplings and not the bi-linear terms which mix the leptons and gauginos; a recent review of these models can be found in [ $[0]$ ]. However we do include the possibility that more than one of the $R_{\mathrm{p}}$ couplings can be non-zero. All the two-body squark and slepton and three-body gaugino and gluino decay modes, and resonant production processes in hadron-hadron collisions are included, as well as a range of production processes in $e^{+} e^{-}$collisions. A decay matrix element is implemented for the threebody decays. The colour structure of these events is very different from that of the MSSM [3. means that a new subroutine HWBRCN is required to handle the colour connections be- 
tween jets in this case. A full discussion of the colour connections in these processes and the matrix elements for the cross sections and decays can be found in $\left[\underline{\bar{p}} \bar{p}_{1}\right]$.

\subsubsection{Related changes}

A large number of changes have been made in HERWIG to enable SUSY processes to be included in hadron-hadron collisions. The main changes are:

- The subroutine HWDHQK has been replaced by HWDHOB which does both heavy quark and SUSY particle decays.

- The subroutines HWBCON, HWCGSP and HWCFOR have been adapted to handle the colour connections found in normal SUSY decays.

- The subroutine HWBRCN has been included to deal with the inter-jet colour connections arising in $R_{\mathrm{p}}$ SUSY. Also HWCBVI, HWCBVT and HWCBCT have been added to handle the hadronization of baryon number violating ( $B$ ) SUSY decays and processes. If the variable RPARTY=.TRUE. (default) then the old HWBCON colour connection code is used, else the new HWBRCN.

\subsection{Hadronization}

For a general hard process in hadron-hadron collisions, we have to consider: $(a)$ the representation of the incoming partons as constituents of the incident hadrons; $(b)$ the conversion of the emitted partons into outgoing hadrons; $(c)$ the "underlying soft event' associated with the presence of spectator partons.

The first of these is dealt with through the use of non-perturbative parton distribution functions, which are discussed below in section 'A. $\overline{1} . \overline{1}$, , and by the remnant hadronization model. The cluster model for hadron formation, remnant hadronization and the underlying event is as follows.

\subsubsection{Cluster model}

The preconfinement property mentioned in section ${ }_{-1}^{1}$ is used by HERWIG as the basis for a simple hadronization model which is local in colour and independent of the

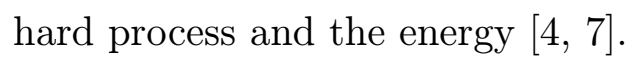

After the perturbative parton showering, all outgoing gluons are split non-perturbatively, into light quark-antiquark or diquark-antidiquark pairs (the default option is to disallow diquark splitting). At this point, each jet consists of a set of outgoing quarks and antiquarks (also possibly some diquarks and antidiquarks) and, in the case of spacelike jets, a single incoming valence quark or antiquark. The latter is replaced by an outgoing spectator carrying the opposite colour and the residual flavour and momentum of the corresponding beam hadron.

In the limit of a large number of colours, each final-state colour line can now be followed from a quark/anti-diquark to an antiquark/diquark with which it can 
form a colour-singlet cluster. ${ }^{5}$ By virtue of pre-confinement, these clusters have a distribution of mass and spatial size that peaks at low values, falls rapidly for large cluster masses and sizes, and is asymptotically independent of the hard subprocess type and scale.

The clusters thus formed are fragmented into hadrons. If a cluster is too light to decay into two hadrons, it is taken to represent the lightest single hadron of its flavour. Its mass is shifted to the appropriate value by an exchange of 4-momentum with a neighbouring cluster in the jet. Similarly, any diquark-antidiquark clusters with masses below threshold for decay into a baryon-antibaryon pair are shifted to the threshold via a transfer of 4-momentum to a neighbouring cluster.

Those clusters massive enough to decay into two hadrons, but below a fission threshold to be specified below, decay isotropically ${ }^{6}$ into pairs of hadrons selected in the following way. A flavour $f$ is chosen at random from among $u, d, s$, the six corresponding diquark flavour combinations, and $c$. For a cluster of flavour $f_{1} \bar{f}_{2}$, this specifies the flavours $f_{1} \bar{f}$ and $f \bar{f}_{2}$ of the decay products, which are then selected at random from tables of hadrons of those flavours. See section for details of the hadrons included. The selected choice of decay products is accepted in proportion to the density of states (phase space times spin degeneracy) for that channel. Otherwise, $f$ is rejected and the procedure is repeated.

The above method of selection for cluster decays is simple and fast but does not automatically satisfy constraints such as strong isospin symmetry. The decay rate into hadrons of a certain flavour depends on the average phase space for channels involving that flavour. Thus, for example, the existence of the $\eta$ or $\eta^{\prime}$, with the same quark content as the $\pi^{0}$, leads to a slight reduction of direct $\pi^{0}$ production relative to $\pi^{+}$and $\pi^{-}$. Quantitatively, the effect is too small to be observed even with the high statistics of the LEP1 data. However, the method can give rise to strange effects if the particle data tables are extended, and modifications to avoid this have been proposed [풀ㄹㄹ.

In the decays of clusters to $\eta$ or $\eta^{\prime}$, the parameter ETAMIX gives the $\eta_{8} / \eta_{0}$ mixing angle in degrees (default $=-20$ ). Rates are not very sensitive to its exact value, as the $\eta^{\prime} / \eta$ suppression is dominated by mass effects in the cluster model. See section for more details.

A fraction of clusters have masses too high for isotropic two-body decay to be a reasonable ansatz, even though the cluster mass spectrum falls rapidly (faster than any power) at high masses. These are fragmented using an iterative fission model until the masses of the fission products fall below the fission threshold. In the fission model the produced flavour $f$ is limited to $u, d$ or $s$ and the product clusters $f_{1} \bar{f}$ and

\footnotetext{
${ }^{5}$ The situation when baryon number is violated is more complicated and is discussed in [4 $\left.{ }_{1}^{-1}\right]$ for the Standard Model and in [399'] for R-parity violating SUSY models.

${ }^{6}$ Except for those containing a 'perturbative' quark when CLDIR $=1$ - see below.
} 
$f \bar{f}_{2}$ move in the directions of the original constituents $f_{1}$ and $\bar{f}_{2}$ in their c.m. frame. Thus the fission mechanism is not unlike string fragmentation [i $\left.{ }_{1}^{4} \overline{3}\right]$ ].

In HERWIG there are three main fission parameters, CLMAX, CLPOW and PSPLT. The maximum cluster mass parameter CLMAX and CLPOW specify the fission threshold $M_{f}$ according to the formula

$$
M_{f}^{\mathrm{CLPOW}}=\mathrm{CLMAX}^{\mathrm{CLPOW}}+\left(m_{1}+m_{2}\right)^{\mathrm{CLPOW}},
$$

where $m_{1}$ and $m_{2}$ are the quark mass parameters $\operatorname{RMASS}(i)$ for flavours $f_{1}$ and $f_{2}$ (see section 'is.2. clusters, which is taken to be $M^{\text {PSPLT }}$ within the allowed phase space. Provided the parameter CLMAX is not chosen too small (the default value is $3.35 \mathrm{GeV}$ ), the gross features of events are insensitive to the details of the fission model, since only a small fraction of clusters undergo fission. However, the production rates of high- $p_{t}$ or heavy particles (especially baryons) are affected, because they are sensitive to the tail of the cluster mass distribution. The default value of the power CLPOW is 2. Smaller values will increase the yield of heavier clusters (and hence of baryons) for heavy quarks, without affecting light quarks much. For example, the default value gives no $b$-baryons (for the default value of CLMAX) whereas CLPOW $=1.0$ makes the ratio of $b$-baryons to $b$-hadrons about $1 / 4$.

There is also a switch CLDIR for cluster decays. If CLDIR $=1$ (the default) then a cluster that contains a 'perturbative' quark, i.e. one coming from the perturbative stage of the event (the hard process or perturbative gluon splitting) 'remembers' its direction. Thus when the cluster decays, the hadron carrying its flavour continues in the same direction (in the cluster c.m. frame) as the quark. This considerably hardens the spectrum of heavy hadrons, particularly of $c$ - and $b$-flavoured hadrons. It also introduces a tendency for baryon-antibaryon pairs preferentially to align themselves with the event axis (the 'TPC $/ 2 \gamma$ string effect' [4] tion, treating clusters containing quarks of perturbative and non-perturbative origin equivalently. In the CLDIR $=1$ option, the parameter CLSMR (default $=0.0$ ) allows for a gaussian smearing of the direction of the perturbative quark's momentum. The smearing is actually exponential in $1-\cos \theta$ with mean CLSMR. Thus increasing CLSMR decorrelates the cluster decay from the initial quark direction.

The process of $b$-quark hadronization requires special treatment and the results obtained using HERWIG are still not fully satisfactory. Generally speaking, it is difficult to obtain a sufficiently hard B-hadron spectrum and the observed $b$-meson $/ b$ baryon ratio. These depend not only on the perturbative subprocess and parton shower but also on non-perturbative issues such as the fraction of $b$-flavoured clusters that become a single $\mathrm{B}$ meson, the fractions that decay into a $\mathrm{B}$ meson and another meson, or into a $b$-baryon and an antibaryon, and the fraction that are split into more clusters. Thus the properties of $b$-jets depend on the parameters RMASS (5), CLMAX, 
CLPOW and PSPLT in a rather complicated way. In practice these parameters are tuned to global final-state properties and one needs extra parameters to describe $b$-jets.

A parameter B1LIM has therefore been introduced to allow clusters somewhat above the $\mathrm{B} \pi$ threshold mass $M_{\mathrm{th}}$ to form a single $\mathrm{B}$ meson if

$$
M<M_{\lim }=(1+\mathrm{B} 1 \mathrm{LIM}) M_{\mathrm{th}} .
$$

The probability of such single-meson clustering is assumed to decrease linearly for $M_{\mathrm{th}}<M<M_{\text {lim }}$. This has the effect of hardening the B spectrum if B1LIM is increased from the default value of zero. In addition, in version 6 , the parameters PSPLT, CLDIR and CLSMR have been converted into two-dimensional arrays, with the first element controlling clusters that do not contain a $b$-quark and the second those that do. Thus tuning of $b$-fragmentation can now be performed separately from other flavours, by setting CLDIR(2) $=1$ and varying PSPLT (2) and CLSMR (2). By reducing the value of PSPLT (2), further hardening of the B-hadron spectrum can be achieved.

\subsubsection{Underlying soft event}

In hadron-hadron and lepton-hadron collisions there are 'beam clusters' containing the spectators from the incoming hadrons. In the formation of beam clusters, the colour connection between the spectators and the initial-state parton showers is cut by the forced emission of a soft quark-antiquark pair. The underlying soft event in a hard hadron-hadron collision is then assumed to be a soft collision between these two beam clusters. In a lepton-hadron collision the corresponding 'soft hadronic remnant' is represented by a soft collision between the beam cluster and the adjacent cluster, i.e. the one produced by the forced emission mentioned above.

The model used for the underlying event is based on the minimum-bias $p \bar{p}$ event generator of the UA5 Collaboration [품] , modified to make use of our cluster fragmentation algorithm. This model is explained in the following subsection.

Adding 10000 to the HERWIG process code IPROC suppresses the underlying event, in which case the beam clusters are simply fragmented like other clusters, without any soft collision. The parameter PRSOF enables one to produce an underlying event in only a fraction PRSOF of events (default $=1.0$ ). Adding 10000 to IPROC is thus equivalent to setting PRSOF $=0$.

A parameter BTCLM is available to users to adjust the mass parameter equivalent to PMBM1 (see below) in remnant cluster formation. Its default value, 1.0, is identical to previous versions. There is also an option for the special treatment of the splitting of clusters containing hadron (or photon) remnants. IOPREM $=0$ gives the fragments a gaussian mass spectrum typical of soft processes. When IOPREM $=1$ (default), the child containing the remnant is treated as before but the other cluster, containing a perturbative parton, is treated as a normal cluster, with mass spectrum $M^{\text {PSPLT }}$.

Two special remnant 'particles' have been defined: 'REMG ' with IDHW $=71$, IDHEP $=98$ and 'REMN ' with IDHW $=72$, IDHEP $=99$. These are remnant pho- 


\begin{tabular}{|l|l|r|}
\hline Name & Description & Default \\
\hline & & 9.110 \\
PMBN1 & $a$ in $\bar{n}=a s^{b}+c$ & 0.115 \\
PMBN2 & $b$ in $\bar{n}=a s^{b}+c$ & -9.500 \\
PMBN3 & $c$ in $\bar{n}=a s^{b}+c$ & \\
& & 0.029 \\
PMBK1 & $a$ in $1 / k=a \ln s+b$ & -0.104 \\
PMBK2 & $b$ in $1 / k=a \ln s+b$ & 0.4 \\
& & 2.0 \\
PMBM1 & $a$ in $\left(M-m_{1}-m_{2}-a\right) e^{-b M}$ & \\
PMBM2 & $b$ in $\left(M-m_{1}-m_{2}-a\right) e^{-b M}$ & 5.2 \\
& & 3.0 \\
PMBP1 & $p_{t}$ slope for $d, u$ & 5.2 \\
PMBP2 & $p_{t}$ slope for $s, c$ & \\
PMBP3 & $p_{t}$ slope for $q q$ & \\
& & \\
\hline
\end{tabular}

Table 5: Soft/min.bias parameters.

tons and nucleons respectively. They are identical to photons and nucleons, except that gluons are labelled as valence partons and, for the nucleon, valence quark distributions are set to zero. They are used by an external package for simulating

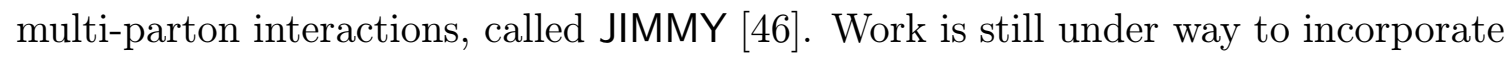
this fully into HERWIG.

\subsubsection{Minimum bias processes}

The minimum-bias event generator of the UA5 Collaboration [4] parametrization of the $p \bar{p}$ inelastic charged multiplicity distribution as a negative binomial distribution. In HERWIG version 6 , the relevant parameters are made available to the user for modification, the default values being the UA5 ones as used in previous versions. These parameters are given in table

The first three parameters control the mean charged multiplicity $\bar{n}$ at c.m. energy $\sqrt{s}$ as indicated. The next two specify the parameter $k$ in the negative binomial charged multiplicity distribution,

$$
P(n)=\frac{\Gamma(n+k)}{n ! \Gamma(k)} \frac{(\bar{n} / k)^{n}}{(1+\bar{n} / k)^{n+k}} .
$$

The parameters PMBM1 and PMBM2 describe the distribution of cluster masses $M$ in the soft collision. These soft clusters are generated using a flat rapidity distribution with gaussian shoulders. The transverse momentum distribution of soft clusters has the form

$$
P\left(p_{t}\right) \propto p_{t} \exp \left(-b \sqrt{p_{t}^{2}+M^{2}}\right)
$$


where the slope parameter $b$ depends as indicated on the flavour of the quark or diquark pair created when the cluster was produced. As an option, for underlying events the value of $\sqrt{s}$ used to choose the multiplicity $n$ may be increased by a factor ENSOF to allow for an enhanced underlying activity in hard events. The actual charged multiplicity is taken to be $n$ plus the sum of the moduli of the charges of the colliding hadrons or clusters.

There is now also an interface to the multiple-interaction model JIMMY [ [ $\left.{ }_{1}^{4} \overline{6} \mathbf{6}\right]$. For this purpose, several routines have been added or modified. New are HWHREM for identifying and cleaning up the beam remnants and HWHSCT to administer the extra scatters. Minor modifications to HWBGEN and HWSBRN suppress energy conservation errors when ISLENT $=-1$; HWSSPC has an improved approximation for remnant mass at high energies; and HWUPCM improves safety against negative square roots.

\subsection{Spacetime structure}

The space-time structure of events is available for all types of subprocess. The production vertex of each parton, cluster, unstable resonance and final-state particle is supplied in the VHEP array of /HEPEVT/. Set PRVTX $=$.TRUE. to include this information when printing the event record (120 column format). The units are: $x, y, z$ in $\mathrm{mm}$ and $t$ in $\mathrm{mm} / c$. In the case of partons and clusters the production points are always given in a local coordinate system with its origin at the relevant hard subprocess. This helps to separate the fermi-scale partonic showers from millimetre-scale distances possible in particle decays, for example the partonic decays of heavy $(c, b)$ hadrons. The vertices of hadrons produced in cluster decays are always corrected back into the laboratory coordinate system.

It is possible to vary the principal interaction point, assigned to the c.m. frame entry in /HEPEVT/ (with ISTHEP $=103$ ), by setting PIPSMR $=$.TRUE. The smearing is generated by the routine HWRPIP according to a triple gaussian given by parameters $\operatorname{VIPWID}(\mathrm{I})$ ( $\mathrm{I}=1,2,3$ for $x, y, z$ widths): the default values correspond to LEP1.

It is also possible to veto particle decays that would occur outside a specified volume by setting MAXDKL = .TRUE. Each putative decay is tested in HWDXLM and if the particle would have decayed outside the chosen volume it is frozen and labelled as final state. Using IOPDKL $=1,2$ selects a cylindrical or spherical allowed region (centred about the origin): then parameters DXRCYL, DXZMAX or DXRSPH specify the dimensions of the region.

\subsubsection{Particle decays}

Lepton and hadron lifetimes (in seconds) are supplied in the array RLTIM. In the case of MSSM (s)particles, including Higgs states, RLTIM values are entered through the input files (see discussion in section 3.5.2i). The lifetimes of heavy quarks (top and any hypothetical extra generations) and weak bosons (including the SM Higgs) are derived from their calculated or specified widths in HWUDKS, whilst light quarks 
and gluons are given an effective minimum width that acts as a lifetime cutoff - see below. All particles whose lifetimes are larger than PLTCUT are set stable.

The proper (i.e. rest-frame) time $t^{*}$ at which an unstable lepton or hadron decays is generated according to the exponential decay law with mean lifetime $\left\langle t^{*}\right\rangle=\tau \equiv$ RLTIM:

$$
\operatorname{Prob}\left(\text { proper time }>t^{*}\right)=\exp \left(-t^{*} / \tau\right)
$$

The laboratory-frame decay time $t$ and distance travelled $d$ are obtained by applying a boost: $t=\gamma t^{*}, d=\beta \gamma t^{*}$ where $\beta=v / c$ and $\gamma=1 / \sqrt{1-\beta^{2}}$. The production vertices of the daughter particles are then calculated by adding the distance travelled by the mother particle as given above to its production vertex. The mean lifetime $\tau$ of a particle is set, taking into account its width and virtuality, by:

$$
\tau\left(q^{2}\right)=\frac{\hbar \sqrt{q^{2}}}{\sqrt{\left(q^{2}-M^{2}\right)^{2}+\left(\Gamma q^{2} / M\right)^{2}}} .
$$

This formula is used for all particles: light partons; heavy quarks and weak bosons, which have appreciable widths; resonances; and unstable leptons. It interpolates between $\tau=\hbar / \Gamma$ for a particle that is on mass-shell and $\tau=\hbar \sqrt{q^{2}} /\left(q^{2}-M^{2}\right)$ for one that is far off mass-shell.

\subsubsection{Parton showers}

The above prescription, based on an exponential proper lifetime distribution, is also used to describe parton showers. For light quarks and gluons, whose natural widths are small, this could lead to unreasonably large distances being generated in the final, low virtuality steps of showering. To avoid this they are given a width $\Gamma=\operatorname{vMIN2} / M$; the parameter VMIN2 (default value $0.1 \mathrm{GeV}^{2}$ ) acts effectively as a lower limit on a parton's virtuality. This is particularly important for the forced splitting of gluons (see section $3 . \overline{6} \cdot \overline{1}$ ), which uses $\tau=\hbar \operatorname{RMASS}(13) /$ VMIN2.

\subsubsection{Hadronization}

In the case of a cluster its initial production vertex is taken as the midpoint of a line perpendicular to the cluster's direction of travel and with its two ends on the trajectories of the constituent quark-antiquark pair. If such a cluster undergoes a forced splitting to two clusters the string picture is adopted. The vertex of the light quark pair is positioned so that the masses of the two daughter clusters would be the same as those for two equivalent string fragments. The production vertices of the daughter clusters are given by the first crossing of their constituent $q \bar{q}$ pairs. The production positions of primary hadrons from cluster decays are smeared, relative to the cluster position, according to a gaussian distribution of width $1 /$ (cluster mass). 


\subsubsection{Colour rearrangement}

HERWIG version 6 contains a colour rearrangement model based on the space-time structure of an event at the end of the parton shower. This is illustrated in the simple example shown below where showering results in a colour-neutral $q g g \bar{q}$ final state. In the conventional HERWIG hadronization model (corresponding to the default value of the reconnection parameter, CLRECO $=$.FALSE.), after a non-perturbative splitting of the final-state gluons, colour singlet clusters are formed from neighbouring $q \bar{q}$ pairs: $(i j)(p q)(k l)$. However when CLRECO $=$.TRUE. the program first creates colour singlet clusters as normal but then checks all (non-neighbouring) pairs of clusters to test if a colour rearrangement lowers the sum of the clusters' spatial sizes added in quadrature. A cluster's size $d_{i j}$ is defined to be the Lorentz-invariant space-time distance between the production points of its constituent quark $q_{i}$ and antiquark $\bar{q}_{j}$. If an allowed alternative is found, that is, $(i j)(k l) \rightarrow(i l)(j k)$ such that $\left|d_{i j}\right|^{2}+\left|d_{k l}\right|^{2}>$ $\left|d_{i l}\right|^{2}+\left|d_{k l}\right|^{2}$, then it is accepted with a probability given by the parameter PRECO (default value $1 / 9$ ).

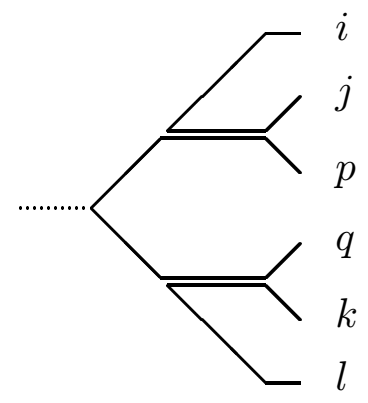

Note that not all colour rearrangements are allowed, for instance in the example shown $(i j)(p q) \rightarrow(i q)(j p)$ is forbidden since the cluster $(j p)$ is a colour octet - it contains both products from a non-perturbative gluon splitting.

Multiple colour rearrangements are considered by the program, as are those between clusters in jets arising from a single, colour neutral source, for example $Z^{0}$ decay (as shown above), or due to more than one source, for example $e^{+} e^{-} \rightarrow$ $W^{+} W^{-} \rightarrow 4$ jets. In the latter case a new parameter, EXAG, is available to exaggerate the lifetime of the $W^{ \pm}$or any other weak boson, so that any dependence of rearrangement effects on source separation can be investigated.

The CLRECO option can be used for all the processes available in HERWIG. Note, however, that before using the program with CLRECO $=$.TRUE. for detailed physics analyses the default parameters should be retuned to LEP data with this option switched on.

\subsubsection{B- $\overline{\mathrm{B}}$ mixing}

When MIXING = .TRUE., particle-antiparticle mixing for $\mathrm{B}_{d, s}^{0}$ mesons is implemented. The probability that a meson is mixed when it decays is given in terms of its lab-frame 


\begin{tabular}{|c|l|c|}
\hline Name & Description & Default \\
\hline PART1 & Type of particle in beam 1 & 'PBAR ' \\
PART2 & Type of particle in beam 2 & 'P ' \\
PBEAM1 & Momentum of beam 1 & 900.0 \\
PBEAM2 & Momentum of beam 2 & 900.0 \\
IPROC & Type of process to generate & 1500 \\
MAXEV & Number of events to generate & 100 \\
\hline
\end{tabular}

Table 6: Main program variables.

decay time $t$ by:

$$
P_{\text {mix }}(t)=\frac{1}{2}-\frac{\cos (X t m / c \tau E)}{2 \cosh (Y t m / c \tau E)},
$$

where $X=\Delta M / \Gamma, Y=\Delta \Gamma / 2 \Gamma$ and $m, \tau, E$ are the $\mathrm{B}^{0}$ mass, lifetime and energy. The ratios $X$ and $Y$ are stored in XMIX(I) and YMIX(I), I $=1,2$ for $q=s, d$. Whenever a neutral B meson occurs in an event, a copy of the original entry is always added to the event record, with ISTHEP $=200$, which gives the particle's flavour at the production (or cluster decay) time. This is in addition to the usual decaying particle entry with ISTHEP $=199$.

\section{Processes}

\subsection{Beams}

As indicated in table ${ }^{6} \cdot$, a number of variables must be set in the main program HWIGPR to specify what is to be simulated. The beam particle types PART1, PART2 can take any of the values NAME listed in table ini.

In the case of point-like photon/QCD processes, IPROC $=5000-5999$, the first particle must be the photon or a lepton. In addition, beams ' $\mathrm{K}+$ ' and ' $\mathrm{K}-$ ' , are supported for minimum bias non-diffractive soft hadronic events $($ IPROC $=8000)$ only. In the case that the beam momenta PBEAM1 and PBEAM2 are not equal, the default procedure (USECMF = .TRUE.) is to generate events in the beambeam centre-of-mass frame and boost them back to the laboratory frame afterwards.

In hadronic processes with lepton beams

\begin{tabular}{|c|c|c|c|}
\hline & NAME & & NAME \\
\hline$e^{+}$ & 'E+' & $e^{-}$ & 'E-' \\
\hline$\mu^{+}$ & 'MU+ ' & $\mu^{-}$ & 'MU- ' \\
\hline$\nu_{e}$ & 'NU_E' & $\bar{\nu}_{e}$ & 'NU_EBAR ' \\
\hline$\nu_{\mu}$ & 'NU_MU ' & $\bar{\nu}_{\mu}$ & 'NU_MUBAR' \\
\hline$\nu_{\tau}$ & 'NU_TAU ' & $\bar{\nu}_{\tau}$ & 'NU_TAUBR' \\
\hline$p$ & 'P ' & $\bar{p}$ & 'PBAR ' \\
\hline$n$ & 'N , & $\bar{n}$ & 'NBAR ' \\
\hline$\pi^{+}$ & 'PI+ ' & $\pi^{-}$ & 'PI- ' \\
\hline$\gamma$ & 'GAMMA ' & & \\
\hline
\end{tabular}

Table 7: Beam particles. (e.g. photoproduction in $e p$ ), the lepton $\rightarrow$ lepton + photon vertex uses the full transverse-momentum dependent splitting function, with exact light-cone kinematics, i.e the Equivalent Photon Approximation 
(EPA). This means that the photon-hadron collision has a transverse momentum in the lepton-hadron frame and must be boosted to a frame where it has no transverse momentum. Thus the c.m.f. boost described above is always used in these processes, regardless of the value of USECMF. The correct lower energy cutoff appropriate to the hadronic process is applied to the photon. The $Q^{2}$ of the photon is generated within the kinematically allowed limits, or the user-defined limits Q2WWMN and Q2WWMX (defaults 0 and 4 ) whichever is more restrictive. ${ }^{7}$ Similarly for the photon's light-cone momentum fraction, with user-defined limits YWWMIN and YWWMAX (default 0 and 1). Together with the Bjorken $y$-variable limits YBMIN and YBMAX, this allows different ranges for the tagged and untagged photons in two-photon DIS.

\subsubsection{Parton distributions}

The parton momentum fraction distributions of the beam particles are used in the generation of initial-state parton showers and also in the non-perturbative process of linking the shower with the beam hadron and its remnant. Since the parton showering is done in leading-logarithmic order, there is no strong motivation to use next-to-leading order parton distributions, although this has become customary since the most up-to-date distributions are deduced from next-to-leading order fits to (inclusive) data. Thus the most common option is to use the interface to the PDFLIB parton distribution library [푹ㄱ.

The HERWIG interface is compatible with PDFLIB version 4 . AUTPDF should be set to the author group as listed in the PDFLIB manual, e.g. 'MRS', and MODPDF to the set number in the new convention. It is permissible to choose the PDFLIB set independently for each of the two beams. For example, to use MRS D- for the proton and Gordon-Storrow set 1 for the photon in $\gamma$-hadron or lepton-hadron collisions, one sets:

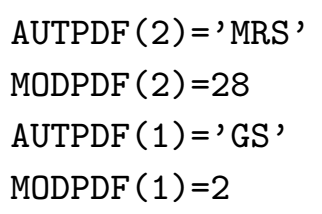

If the PDFLIB interface is not used, the default parton distributions for hadrons are the leading-order Owens set $1.1[\overline{4} \overline{8} \overline{\mathrm{B}}]$ (NSTRU = 5), similar to Duke and Owens 1, but fitted to more recent data. For photons, the default is to use the Drees-Grassie distributions [4] $\overline{4}$ ]. The heavy quark content of the photon uses the corrections to the Drees-Grassie distribution functions for light quarks, calculated by Drees and Kim [i $[\overline{0} \overline{0}]$. There is

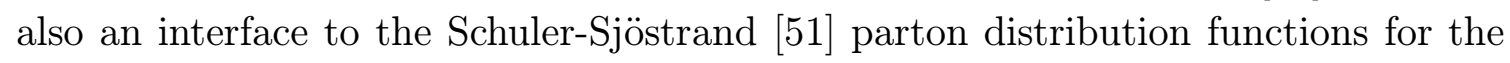
photon, version 2. These appear as PDFLIB sets with author group 'SaSph', but are actually implemented via a call to their SASGAM code. The value in MODPDF specifies the set (1-4 for 1D [recommended set],1M, 2D,2M), whether the Bethe-Heitler process

\footnotetext{
${ }^{7}$ The WW in parameter names is a relic from earlier versions that used the less accurate WeizsackerWilliams approximation.
} 
is used for heavy flavours (add 10), whether the $P^{2}$-dependence is included (add 20), and which of their $P^{2}$ models is used (add 100 times their IP2 parameter).

An option to damp the parton distributions of off mass-shell photons relative to on-shell photons, according to the scheme of Drees and Godbole [52] has been introduced. The adjustable parameter PHOMAS defines the crossover from the nonsuppressed to suppressed regimes. Recommended values lie in the range from QCDLAM to $1 \mathrm{GeV}$. The default value PHOMAS $=0$ corresponds to no suppression, as in previous versions.

\subsection{Summary of subprocesses}

We give in table ${ }_{-1}^{8}$ a list of the currently available hard subprocesses IPROC. More detailed descriptions are given in sections 'A are instructions to users on how to add a new process.

\begin{tabular}{|c|c|}
\hline IPROC & Process \\
\hline 100 & $\ell^{+} \ell^{-} \rightarrow q \bar{q}(g)$ (all $q$ flavours) \\
\hline $100+\mathrm{IQ}$ & $\ell^{+} \ell^{-} \rightarrow q \bar{q}(g)(\mathrm{IQ}=1,2,3,4,5,6$ for $q=d, u, s, c, b, t)$ \\
\hline 107 & $\ell^{+} \ell^{-} \rightarrow g g(g)$ (fictitious process) \\
\hline 110 & $\ell^{+} \ell^{-} \rightarrow q \bar{q} g$ (all flavours) \\
\hline $110+\mathrm{IQ}$ & $\ell^{+} \ell^{-} \rightarrow q \bar{q} g(\mathrm{IQ}$ as above $)$ \\
\hline 120 & $\ell^{+} \ell^{-} \rightarrow q \bar{q}$ (all flavours, no hard gluon correction) \\
\hline $120+\mathrm{IQ}$ & $\ell^{+} \ell^{-} \rightarrow q \bar{q}$ (IQ as above, no hard gluon correction) \\
\hline 127 & $\ell^{+} \ell^{-} \rightarrow g g$ (fictitious process, no hard gluon correction) \\
\hline $150+\mathrm{IL}$ & $\ell^{+} \ell^{-} \rightarrow \ell^{\prime} \bar{\ell}^{\prime}\left(\mathrm{IL}=1,2,3\right.$ for $\ell^{\prime}=e, \mu, \tau$, N.B. $\left.\ell \neq \ell^{\prime}\right)$ \\
\hline 200 & $\ell^{+} \ell^{-} \rightarrow W^{+} W^{-}$(see sect. 'IA.3. $\overline{2}_{-1}^{-}$on control of $W / Z$ decays) \\
\hline 250 & $\ell^{+} \ell^{-} \rightarrow Z^{0} Z^{0}$ (see sect. 'A.3 on control of $W / Z$ decays) \\
\hline 300 & $\ell^{+} \ell^{-} \rightarrow Z^{0} H_{\mathrm{SM}}^{0} \rightarrow Z^{0} q \bar{q}$ (all flavours) \\
\hline $300+\mathrm{IQ}$ & $\ell^{+} \ell^{-} \rightarrow Z^{0} H_{\mathrm{SM}}^{0} \rightarrow Z^{0} q \bar{q}(\mathrm{IQ}$ as above $)$ \\
\hline $306+\mathrm{IL}$ & $\ell^{+} \ell^{-} \rightarrow Z^{0} H_{\mathrm{SM}}^{0} \rightarrow Z^{0} \ell \bar{\ell}($ IL as above $)$ \\
\hline 310,311 & $\ell^{+} \ell^{-} \rightarrow Z^{0} H_{\mathrm{SM}}^{0} \rightarrow Z^{0} W^{+} W^{-}, Z^{0} Z^{0} Z^{0}$ \\
\hline 312 & $\ell^{+} \ell^{-} \rightarrow Z^{0} H_{\mathrm{SM}}^{0} \rightarrow Z^{0} \gamma \gamma$ \\
\hline 399 & $\ell^{+} \ell^{-} \rightarrow Z^{0} H_{\mathrm{SM}}^{0} \rightarrow Z^{0}$ anything \\
\hline $400+\mathrm{ID}$ & $\ell^{+} \ell^{-} \rightarrow \nu \bar{\nu} H_{\mathrm{SM}}^{0}+\ell^{+} \ell^{-} H_{\mathrm{SM}}^{0}($ ID as in IPROC $=300+\mathrm{ID})$ \\
\hline $500+\mathrm{ID}$ & $\begin{array}{l}\ell^{+} \ell^{-} \rightarrow \ell^{+} \ell^{-} \gamma \gamma \rightarrow \ell^{+} \ell^{-} q \bar{q} / \ell \bar{\ell} / W^{+} W^{-} \\
\quad(\mathrm{ID}=0-10 \text { as in IPROC }=300+\text { ID })\end{array}$ \\
\hline $550+$ ID & $\ell^{+} \ell^{-} \rightarrow \ell \nu_{\ell} \gamma W \rightarrow \ell \nu_{\ell} q \bar{q}^{\prime} / \ell \bar{\ell}^{\prime}(\mathrm{ID}=0-9$ as in IPROC $=300+\mathrm{ID})$ \\
\hline 600 & $\ell^{+} \ell^{-} \rightarrow q \bar{q} g g, q \bar{q} q^{\prime} \bar{q}^{\prime}$ (all $q$ flavours) \\
\hline $600+\mathrm{IQ}$ & $\ell^{+} \ell^{-} \rightarrow q \bar{q} g g, q \bar{q} q^{\prime} \bar{q}^{\prime}$ (IQ as above) \\
\hline & After generation, IHPRO is subprocess (see sect. 'A.3.5') \\
\hline $\begin{array}{c}700-99 \\
700\end{array}$ & $\begin{array}{l}\text { Minimal Supersymmetric Standard Model (MSSM) processes } \\
\ell^{+} \ell^{-} \rightarrow 2 \text {-sparticle processes (sum of } 710,730,740 \text { and } 760 \text { ) }\end{array}$ \\
\hline
\end{tabular}

Table 18:; Process codes. (Continues) 


\begin{tabular}{|c|c|}
\hline IPROC & Process \\
\hline 710 & $\ell^{+} \ell^{-} \rightarrow$ neutralino pairs (all neutralinos) \\
\hline $706+4 \mathrm{IN} 1+\mathrm{IN} 2$ & $\ell^{+} \ell^{-} \rightarrow \widetilde{\chi}_{\mathrm{IN} 1}^{0} \widetilde{\chi}_{\mathrm{IN} 2}^{0}(\mathrm{IN} 1,2=$ neutralino mass eigenstate $)$ \\
\hline 730 & $\ell^{+} \ell^{-} \rightarrow$ chargino pairs (all charginos) \\
\hline $728+2 \mathrm{IC} 1+\mathrm{IC} 2$ & $\ell^{+} \ell^{-} \rightarrow \widetilde{\chi}_{\mathrm{IC} 1}^{+} \widetilde{\chi}_{\mathrm{IC} 2}^{-}(\mathrm{IC} 1,2=$ chargino mass eigenstate $)$ \\
\hline 740 & $\ell^{+} \ell^{-} \rightarrow$ slepton pairs (all flavours) \\
\hline $736+5 \mathrm{IL}$ & $\ell^{+} \ell^{-} \rightarrow \widetilde{\ell}_{L, R} \widetilde{\ell}_{L, R}^{*}(\mathrm{IL}=1,2,3$ for $\tilde{\ell}=\tilde{e}, \tilde{\mu}, \tilde{\tau})$ \\
\hline $737+5 \mathrm{IL}$ & $\ell^{+} \ell^{-} \rightarrow \widetilde{\ell}_{L} \widetilde{\ell}_{L}^{*}($ IL as above $)$ \\
\hline $738+5 \mathrm{IL}$ & $\ell^{+} \ell^{-} \rightarrow \tilde{\ell}_{L} \tilde{\ell}_{R}^{*}$ (IL as above) \\
\hline $739+5 \mathrm{IL}$ & $\ell^{+} \ell^{-} \rightarrow \widetilde{\ell}_{R} \widetilde{\ell}_{R}^{*}$ (IL as above) \\
\hline $740+5$ IL & $\ell^{+} \ell^{-} \rightarrow \widetilde{\nu}_{L} \widetilde{\nu}_{L}^{*}\left(\mathrm{IL}=1,2,3\right.$ for $\left.\widetilde{\nu}_{e}, \widetilde{\nu}_{\mu}, \widetilde{\nu}_{\tau}\right)$ \\
\hline 760 & $\ell^{+} \ell^{-} \rightarrow$ squark pairs (all flavours) \\
\hline $757+4 \mathrm{IQ}$ & $\ell^{+} \ell^{-} \rightarrow \widetilde{q}_{L, R} \widetilde{q}_{L, R}^{*}(\mathrm{IQ}=1,2,3,4,5,6$ for $\widetilde{q}=\tilde{d}, \tilde{u}, \tilde{s}, \tilde{c}, \tilde{b}, \tilde{t})$ \\
\hline $758+4 \mathrm{IQ}$ & $\ell^{+} \ell^{-} \rightarrow \widetilde{q}_{L} \widetilde{q}_{L}^{*}(\mathrm{IQ}$ as above $)$ \\
\hline $759+4 \mathrm{IQ}$ & $\ell^{+} \ell^{-} \rightarrow \widetilde{q}_{L} \widetilde{q}_{R}^{*}($ IQ as above $)$ \\
\hline $760+4 \mathrm{IQ}$ & $\ell^{+} \ell^{-} \rightarrow \widetilde{q}_{R} \widetilde{q}_{R}^{*}$ (IQ as above) \\
\hline $800-99$ & R-parity violating supersymmetric processes \\
\hline 800 & Single sparticle production, sum of $810-840$ \\
\hline 810 & $\ell^{+} \ell^{-} \rightarrow \tilde{\chi}^{0} \nu_{i}$, (all neutralinos) \\
\hline $810+\mathrm{IN}$ & $\ell^{+} \ell^{-} \rightarrow \widetilde{\chi}_{\mathrm{IN}}^{0} \nu_{i},(\mathrm{IN}=$ neutralino mass state $)$ \\
\hline 820 & $\ell^{+} \ell^{-} \rightarrow \tilde{\chi}^{-} e_{i}^{+}$(all charginos) \\
\hline $820+\mathrm{IC}$ & $\ell^{+} \ell^{-} \rightarrow \tilde{\chi}_{\mathrm{IC}}^{-} e_{i}^{+},(\mathrm{IC}=$ chargino mass state $)$ \\
\hline 830 & $\ell^{+} \ell^{-} \rightarrow \widetilde{\nu}_{i} Z^{0}$ and $\ell^{+} \ell^{-} \rightarrow \widetilde{\ell}_{i}^{+} W^{-}$ \\
\hline 840 & $\ell^{+} \ell^{-} \rightarrow \widetilde{\nu}_{i} h^{0} / H^{0} / A^{0}$ and $\ell^{+} \ell^{-} \rightarrow \widetilde{\ell}_{i}^{+} H^{-}$ \\
\hline 850 & $\ell^{+} \ell^{-} \rightarrow \widetilde{\nu}_{i} \gamma$ \\
\hline 860 & Sum of 870 and 880 \\
\hline 870 & $\ell^{+} \ell^{-} \rightarrow \ell^{+} \ell^{-}$, via LLE only \\
\hline $867+3$ IL $1+$ IL2 & $\ell^{+} \ell^{-} \rightarrow \ell_{\mathrm{IL} 1}^{+} \ell_{\mathrm{IL} 2}^{-}(\mathrm{IL} 1,2=1,2,3$ for $e, \mu, \tau)$ \\
\hline 880 & $\ell^{+} \ell^{-} \rightarrow \bar{d} d$, via LLE and LQD \\
\hline $877+3 \mathrm{IQ} 1+\mathrm{IQ} 2$ & $\ell^{+} \ell^{-} \rightarrow d_{\mathrm{IL} 1} \bar{d}_{\mathrm{IL} 2}(\mathrm{IQ} 1,2=1,2,3$ for $d, s, b)$ \\
\hline 1300 & $q \bar{q} \rightarrow Z^{0} / \gamma \rightarrow q^{\prime} \bar{q}^{\prime}$ (all flavours) \\
\hline $1300+\mathrm{IQ}$ & $q \bar{q} \rightarrow Z^{0} / \gamma \rightarrow q^{\prime} \bar{q}^{\prime}(\mathrm{IQ}=1,2,3,4,5,6$ for $q=d, u, s, c, b, t)$ \\
\hline 1350 & $q \bar{q} \rightarrow Z^{0} / \gamma \rightarrow \ell \bar{\ell}($ all lepton species) \\
\hline $\begin{array}{l}1350+\mathrm{IL} \\
1399\end{array}$ & $\begin{array}{l}q \bar{q} \rightarrow Z^{0} / \gamma \rightarrow \ell \bar{\ell}\left(\mathrm{IL}=1-6 \text { for } \ell=e, \nu_{e}, \mu, \nu_{\mu}, \text { etc. }\right) \\
q \bar{q} \rightarrow Z^{0} / \gamma \rightarrow \text { anything }\end{array}$ \\
\hline 1400 & $q \bar{q} \rightarrow W^{ \pm} \rightarrow q^{\prime} \bar{q}^{\prime \prime}$ (all flavours) \\
\hline $1400+\mathrm{IQ}$ & $q \bar{q} \rightarrow W^{ \pm} \rightarrow q^{\prime} \bar{q}^{\prime \prime}\left(q^{\prime}\right.$ or $q^{\prime \prime}$ as above $)$ \\
\hline 1450 & $q \bar{q} \rightarrow W^{ \pm} \rightarrow \ell \nu_{\ell}$ (all lepton species) \\
\hline
\end{tabular}

Table '8:- Process codes. (Continues) 


\begin{tabular}{|c|c|}
\hline IPROC & Process \\
\hline $\begin{array}{c}1450+\mathrm{IL} \\
1499\end{array}$ & $\begin{array}{l}q \bar{q} \rightarrow W^{ \pm} \rightarrow \ell \nu_{\ell}(\mathrm{IL}=1,2,3 \text { for } \ell=e, \mu, \tau) \\
q \bar{q} \rightarrow W^{ \pm} \rightarrow \text { anything }\end{array}$ \\
\hline 1500 & $\begin{array}{l}\text { QCD } 2 \rightarrow 2 \text { hard parton scattering } \\
\text { After generation, IHPRO is subprocess (see sect. 'T. } \overline{6} . \overline{2} . \overline{2} \text { ) }\end{array}$ \\
\hline $1600+$ ID & $g g / q \bar{q} \rightarrow H_{\mathrm{SM}}^{0}(\mathrm{ID}$ as in IPROC $=300+$ ID $)$ \\
\hline $1700+\mathrm{IQ}$ & $\begin{array}{l}\text { QCD heavy quark production (IQ as above) } \\
\text { After generation, IHPRO is subprocess (see sect. 'T. }\end{array}$ \\
\hline 1800 & $\begin{array}{l}\text { QCD direct photon }+ \text { jet production } \\
\left.\text { After generation, IHPRO is subprocess (see sect. 'T. } \overline{6} . \overline{y_{1}^{\prime}}\right)\end{array}$ \\
\hline $1900+$ ID & $q \bar{q} \rightarrow q^{\prime} \bar{q}^{\prime} W^{+} W^{-} / Z^{0} Z^{0} \rightarrow q^{\prime} \bar{q}^{\prime} H($ ID as in IPROC $=300+$ ID $)$ \\
\hline $\begin{array}{c}2000 \\
2001-4 \\
2005-8\end{array}$ & $\begin{array}{l}t \text { production via } W^{ \pm} \text {exchange (sum of 2001-2008) } \\
\bar{u} \bar{b} \rightarrow \bar{d} \bar{t}, \quad d \bar{b} \rightarrow u \bar{t}, \quad \overline{d b} \rightarrow \bar{u} \bar{t}, \quad u b \rightarrow d t \\
\bar{c} \bar{b} \rightarrow \bar{s} \bar{t}, \quad s \bar{b} \rightarrow c \bar{t}, \quad \bar{s} b \rightarrow \bar{c} t, \quad c b \rightarrow s t\end{array}$ \\
\hline $\begin{array}{l}2100 \\
2110 \\
2120 \\
2150 \\
2160 \\
2170\end{array}$ & $\begin{array}{l}W^{ \pm}+\text {jet production } \\
W^{ \pm}+\text {jet production (Compton only: } g q \rightarrow W q \text { ) } \\
W^{ \pm}+\text {jet production (annihilation only: } q \bar{q} \rightarrow W g \text { ) } \\
Z^{0}+\text { jet production } \\
Z^{0}+\text { jet production (Compton only: } g q \rightarrow Z q \text { ) } \\
Z^{0}+\text { jet production (snnihilation only: } q \bar{q} \rightarrow Z g \text { ) }\end{array}$ \\
\hline 2200 & $\begin{array}{l}\text { QCD direct photon pair production } \\
\left.\text { After generation, IHPRO is subprocess (see sect. 'A. }{ }^{-} \cdot \bar{b}_{-}^{\prime}\right)\end{array}$ \\
\hline $2300+\mathrm{ID}$ & $\begin{array}{l}\text { QCD SM Higgs }+ \text { jet production (ID as in IPROC }=300+\text { ID) } \\
\text { After generation, IHPRO is subprocess (see sect. 'A. }\end{array}$ \\
\hline $\begin{array}{l}2400 \\
2450\end{array}$ & $\begin{array}{l}\text { Mueller-Tang colour singlet exchange } \\
\text { Quark scattering via photon exchange }\end{array}$ \\
\hline $2500+$ ID & $g g / q \bar{q} \rightarrow t \bar{t} H_{\mathrm{SM}}^{0}(\mathrm{ID}$ as in IPROC $=300+\mathrm{ID})$ \\
\hline $2600+$ ID & $q \bar{q}^{\prime} \rightarrow W^{ \pm} H_{\mathrm{SM}}^{0}($ ID as in IPROC $=300+\mathrm{ID})$ \\
\hline $2700+$ ID & $q \bar{q} \rightarrow Z^{0} H_{\mathrm{SM}}^{0}($ ID as in IPROC $=300+\mathrm{ID})$ \\
\hline $\begin{array}{l}3000-999 \\
3000 \\
3010 \\
3020 \\
3030\end{array}$ & $\begin{array}{l}\text { Minimal Supersymmetric Standard Model (MSSM) processes } \\
2 \text {-parton } \rightarrow 2 \text {-sparticle processes (sum of those below) } \\
2 \text {-parton } \rightarrow 2 \text {-sparton processes } \\
2 \text {-parton } \rightarrow 2 \text {-gaugino processes } \\
\text { 2-parton } \rightarrow 2 \text {-slepton processes }\end{array}$ \\
\hline $\begin{array}{r}3310,3315 \\
3320,3325 \\
3335\end{array}$ & $\begin{aligned} q \bar{q}^{\prime} \rightarrow W^{ \pm} h^{0}, H^{ \pm} h^{0} & \left(\text { all } q, q^{\prime} \text { flavours - gauge bosons mediated only) }\right. \\
& \quad \bar{q}^{\prime} \rightarrow W^{ \pm} H^{0}, H^{ \pm} H^{0} \quad \text { "") } \\
q \bar{q}^{\prime} \rightarrow & H^{ \pm} A^{0}(")\end{aligned}$ \\
\hline
\end{tabular}

Table 18;: Process codes. (Continues) 


\begin{tabular}{|c|c|}
\hline IPROC & Process \\
\hline 3350 & $q \bar{q} \rightarrow W^{ \pm} H^{\mp}$ (Higgstrahlung and Higgs mediated) \\
\hline 3355 & $q \bar{q} \rightarrow H^{ \pm} H^{\mp}$ (all $q$ flavours — gauge boson mediated only) \\
\hline 3360,3365 & $q \bar{q} \rightarrow Z^{0} h^{0}, A^{0} h^{0}(")$ \\
\hline 3370,3375 & $q \bar{q} \rightarrow Z^{0} H^{0}, A^{0} H^{0}(")$ \\
\hline 3410 & $b g \rightarrow b h^{0}+$ ch. conj. \\
\hline 3420 & $b g \rightarrow b H^{0}+$ ch. conj. \\
\hline 3430 & $b g \rightarrow b A^{0}+$ ch. conj. \\
\hline 3450 & $b g \rightarrow t H^{-}+$ch. conj. \\
\hline 3610 & $q \bar{q} / g g \rightarrow h^{0} \quad$ (light scalar Higgs) \\
\hline 3620 & $q \bar{q} / g g \rightarrow H^{0} \quad$ (heavy scalar Higgs) \\
\hline 3630 & $q \bar{q} / g g \rightarrow A^{0}$ (pseudoscalar Higgs) \\
\hline $4000-99$ & R-parity violating supersymmetric processes via LQD \\
\hline 4000 & single sparticle production, sum of $4010-4050$ \\
\hline 4010 & $\bar{u}_{j} d_{k} \rightarrow \widetilde{\chi}^{0} l_{i}^{-}, \bar{d}_{j} d_{k} \rightarrow \widetilde{\chi}^{0} \nu_{i}$ (all neutralinos) \\
\hline $4010+\mathrm{IN}$ & $\bar{u}_{j} d_{k} \rightarrow \widetilde{\chi}_{\mathrm{IN}}^{0} l_{i}^{-}, \bar{d}_{j} d_{k} \rightarrow \widetilde{\chi}_{\mathrm{IN}}^{0} \nu_{i}(\mathrm{IN}=$ neutralino mass state $)$ \\
\hline 4020 & $\bar{u}_{j} d_{k} \rightarrow \tilde{\chi}^{-} \nu_{i}, \bar{d}_{j} d_{k} \rightarrow \tilde{\chi}^{-} e_{i}^{+}$(all charginos) \\
\hline $4020+\mathrm{IC}$ & $\bar{u}_{j} d_{k} \rightarrow \tilde{\chi}_{\mathrm{IC}}^{-} \nu_{i}, \bar{d}_{j} d_{k} \rightarrow \tilde{\chi}_{\mathrm{IC}}^{-} e_{i}^{+}(\mathrm{IC}=$ chargino mass state $)$ \\
\hline 4040 & $u_{j} \bar{d}_{k} \rightarrow \underset{\sim}{\tilde{\tau}_{i}^{+}} Z^{0}, u_{j} \bar{d}_{k} \rightarrow \widetilde{\nu}_{i} W^{+}$and $d_{j} \bar{d}_{k} \rightarrow \widetilde{\ell}_{i}^{+} W^{-}$ \\
\hline 4050 & $u_{j} \bar{d}_{k} \rightarrow \widetilde{\ell}_{i}^{+} h^{0} / H^{0} / A^{0}, u_{j} \bar{d}_{k} \rightarrow \widetilde{\nu}_{i} H^{+}$and $d_{j} \bar{d}_{k} \rightarrow \widetilde{\ell}_{i}^{+} H^{-}$ \\
\hline 4060 & Sum of 4070 and 4080 \\
\hline 4070 & $\bar{u}_{j} d_{k} \rightarrow \bar{u}_{l} d_{m}$ and $\bar{d}_{j} d_{k} \rightarrow \bar{d}_{l} d_{m}$, via LQD only \\
\hline 4080 & $\bar{u}_{j} d_{k} \rightarrow \nu_{j} l_{k}^{-}$and $\bar{d}_{j} d_{k} \rightarrow l_{j}^{+} l_{k}^{-}$, via LQD and LLE \\
\hline $4100-99$ & R-parity violating supersymmetric processes via UDD \\
\hline 4100 & single sparticle production, sum of $4110-4150$ \\
\hline 4110 & $u_{i} d_{j} \rightarrow \tilde{\chi}^{0} \bar{d}_{k}, d_{j} d_{k} \rightarrow \tilde{\chi}^{0} \bar{u}_{i}($ all neutralinos $)$ \\
\hline $4110+\mathrm{IN}$ & $u_{i} d_{j} \rightarrow \widetilde{\chi}_{\mathrm{IN}}^{0} \bar{d}_{k}, d_{j} d_{k} \rightarrow \widetilde{\chi}_{\mathrm{IN}}^{0} \bar{u}_{i}(\mathrm{IN}$ as above $)$ \\
\hline 4120 & $u_{i} d_{j} \rightarrow \tilde{\chi}^{+} \bar{u}_{k}, d_{j} d_{k} \rightarrow \tilde{\chi}^{-} \bar{d}_{i}($ all charginos $)$ \\
\hline $4120+\mathrm{IC}$ & $u_{i} d_{j} \rightarrow \tilde{\chi}_{\mathrm{IC}}^{+} \bar{u}_{k}, d_{j} d_{k} \rightarrow \tilde{\chi}_{\mathrm{IC}}^{-} \bar{d}_{i}(\mathrm{IC}$ as above $)$ \\
\hline 4130 & $u_{i} d_{j} \rightarrow \widetilde{g} \bar{d}_{k}, d_{j} d_{k} \rightarrow \widetilde{g} \bar{u}_{i}$ \\
\hline 4140 & $u_{i} d_{j} \rightarrow \tilde{b}_{1}^{*} Z^{0}, d_{j} d_{k} \rightarrow \tilde{t}_{1}^{*} Z^{0}, u_{i} d_{j} \rightarrow \tilde{t}_{i}^{*} W^{+}$and $d_{j} d_{k} \rightarrow \tilde{b}_{i}^{*} W^{-}$ \\
\hline 4150 & $\begin{array}{r}u_{i} d_{j} \rightarrow \tilde{d}_{k 1}^{*} h^{0} / H^{0} / A^{0}, d_{j} d_{k} \rightarrow \tilde{u}_{i 1}^{*} h^{0} / H^{0} / A^{0}, u_{i} d_{j} \rightarrow \tilde{u}_{k \alpha}^{*} H^{+} \\
d_{j} d_{k} \rightarrow \tilde{d}_{i \alpha}^{*} H^{-}\end{array}$ \\
\hline 4160 & $u_{i} d_{j} \rightarrow u_{l} d_{m}, d_{j} d_{k} \rightarrow d_{l} d_{m}$ via UDD. \\
\hline $4200-99$ & Graviton resonance production \\
\hline 4200 & Sum of 4210,4250 and 4270 \\
\hline 4210 & $g g / q \bar{q} \rightarrow G \rightarrow g g / q \bar{q}$ (all partons) \\
\hline $4210+\mathrm{IQ}$ & $g g / q \bar{q} \rightarrow G \rightarrow q \bar{q}($ IQ as above $)$ \\
\hline 4220 & $g g / q \bar{q} \rightarrow G \rightarrow g g$ \\
\hline
\end{tabular}

Table 18:- Process codes. (Continues) 


\begin{tabular}{|c|c|}
\hline IPROC & Process \\
\hline $\begin{array}{c}4250 \\
4250+\mathrm{IL} \\
4260 \\
4270 \\
4271 \\
4272 \\
4273\end{array}$ & $\begin{array}{l}g g / q \bar{q} \rightarrow G \rightarrow \ell \bar{\ell}(\text { all leptons }) \\
g g / q \bar{q} \rightarrow G \rightarrow \ell \bar{\ell}\left(\mathrm{IL}=1-6 \text { for } \ell=e, \nu_{e}, \mu, \nu_{\mu}, \text { etc. }\right) \\
g g / q \bar{q} \rightarrow G \rightarrow \gamma \gamma \\
g g / q \bar{q} \rightarrow G \rightarrow W^{+} W^{-} / Z^{0} Z^{0} / H_{\mathrm{SM}}^{0} H_{\mathrm{SM}}^{0} \\
g g / q \bar{q} \rightarrow G \rightarrow W^{+} W^{-} \\
g g / q \bar{q} \rightarrow G \rightarrow Z^{0} Z^{0} \\
g g / q \bar{q} \rightarrow G \rightarrow H_{\mathrm{SM}}^{0} H_{\mathrm{SM}}^{0}\end{array}$ \\
\hline $\begin{array}{c}5000 \\
5100+\mathrm{IQ} \\
5200+\mathrm{IQ} \\
\\
5300 \\
5500 \\
5510,20\end{array}$ & 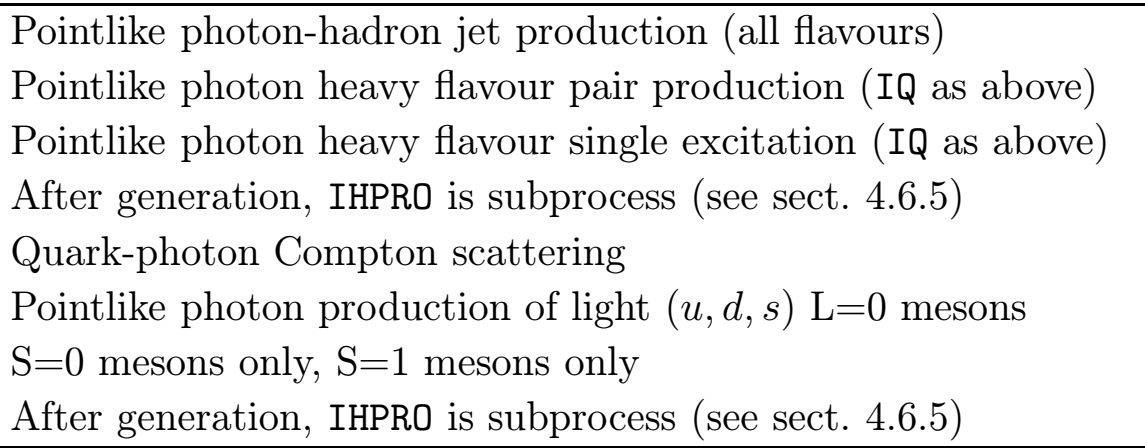 \\
\hline $\begin{array}{c}6000 \\
6000+\mathrm{IQ} \\
6006+\mathrm{IL} \\
6010\end{array}$ & $\begin{array}{l}\gamma \gamma \rightarrow q \bar{q} \text { (all flavours) } \\
\gamma \gamma \rightarrow q \bar{q}(\mathrm{IQ} \text { as above }) \\
\gamma \gamma \rightarrow \ell \bar{\ell}(\mathrm{IL}=1,2,3 \text { for } \ell=e, \mu, \tau) \\
\gamma \gamma \rightarrow W^{+} W^{-}\end{array}$ \\
\hline $\begin{array}{l}7000- \\
7999\end{array}$ & $\begin{array}{l}\text { Baryon-number violating and other multi- } W^{ \pm} \text {processes } \\
\text { generated by HERBVI package }\end{array}$ \\
\hline 8000 & Minimum bias soft hadron-hadron event \\
\hline $\begin{array}{c}9000 \\
9000+\mathrm{IQ} \\
9010 \\
9010+\mathrm{IQ}\end{array}$ & $\begin{array}{l}\text { Deep inelastic lepton scattering (all neutral current) } \\
\text { Deep inelastic lepton scattering (NC on flavour IQ) } \\
\text { Deep inelastic lepton scattering (all charged current) } \\
\text { Deep inelastic lepton scattering (CC on flavour IQ) }\end{array}$ \\
\hline $\begin{array}{c}9100 \\
9100+\mathrm{IQ} \\
9107 \\
9110 \\
9110+\mathrm{IP} \\
9130 \\
9140+\mathrm{IP}\end{array}$ & $\begin{array}{l}\text { Boson-gluon fusion in neutral current DIS (all flavours) } \\
\text { Boson-gluon fusion in neutral current DIS (IQ as above) } \\
J / \psi+\text { gluon production by boson-gluon fusion } \\
\text { QCD Compton process in neutral current DIS (all flavours) } \\
\text { QCD Compton process in NC DIS (IP }=1-12 \text { for } d-t, \bar{d}-\bar{t} \text { ) } \\
\left.\text { All } \mathcal{O}\left(\alpha_{\mathrm{S}}\right) \text { NC processes (i.e. } 9100+9110\right) \\
\text { Heavy quark production by charged-current boson-gluon fusion } \\
\text { IP: } 1=s \bar{c}, 2=b \bar{c}, 3=s \bar{t}, 4=b \bar{t} \text { ( }+ \text { ch. conj.) }\end{array}$ \\
\hline $9500+\mathrm{ID}$ & $W^{+} W^{-} / Z^{0} Z^{0} \rightarrow H_{\mathrm{SM}}^{0}$ in DIS (ID as in IPROC $=300+$ ID $)$ \\
\hline $10000+\mathrm{IP}$ & $\begin{array}{l}\text { as IPROC = IP but with soft underlying event } \\
\text { (soft remnant fragmentation in lepton-hadron) suppressed }\end{array}$ \\
\hline
\end{tabular}

Table i8: Process codes. 


\subsubsection{Treatment of quark masses}

The extent to which quark mass effects are included in the hard process cross section is different in different processes. In many processes, they are always treated as massless: IPROC $=1300,1800,1900,2100,2300,2400,5300,9000$. In two processes they are all treated as massless except the top quark, for which the mass is correctly incorporated: 1400, 2000. In the case of massless pair production, only quark flavours that are kinematically allowed are produced. In all cases the event kinematics incorporate the quark mass, even when it is not used to calculate the cross section. In two processes, quarks are always treated as massive: 500, 9100. Finally, in several processes, the behaviour is different depending on whether a specific quark flavour is requested, in which case its mass is included, or not, in which case all quarks are treated as massless. These are: IPROC $=100,110,120$, QCD $2 \rightarrow 2$ scattering (1500 vs. $1700+\mathrm{IQ}$ ), jets in direct photoproduction (5000 vs. 5100+IQ and 5200+IQ).

These differences can cause inconsistencies between different ways of generating the same process. The most noticeable example is in direct photoproduction, where one can use process 9130, which uses the exact $2 \rightarrow 3$ matrix element $e+g \rightarrow$ $e+q+\bar{q}$, or process 5000, which uses the Equivalent Photon Approximation (EPA) for $e \rightarrow e+\gamma$ and the $2 \rightarrow 2$ matrix element for $\gamma+g \rightarrow q+\bar{q}$. For typical HERA kinematics, the EPA is valid to a few per cent, but the difference between the two processes is much larger, about $20 \%$ for PTMIN $=2 \mathrm{GeV}$. This is entirely due to the difference in quark mass treatments, as can be checked by comparing process 9130 with processes $5100+$ IQ and 5200+IQ summed over IQ.

\subsubsection{Couplings}

The two-loop QCD coupling at scale $Q$ is given by subroutine HWUALF with arguments IOPT $=1$ and SCALE $=Q$. Threshold matching is performed at the quark mass scales $Q=\operatorname{RMASS}(i)$. Setting IOPT $=0$ initializes the coupling using the 5 -flavour value $\Lambda_{\overline{\mathrm{MS}}}=$ QCDLAM. Other values of IOPT are for internal use only.

The electromagnetic coupling is given by $\operatorname{HWUAEM}\left(Q^{2}\right)=e^{2} /(4 \pi)$; it runs according to the prescription in ref. [5 parameter ALPHEM $\equiv \operatorname{HWUAEM}(0)$, default value 0.0072993 , provides the normalisation at the Thomson limit; it is used for all processes involving real photons. Photon emission in parton showers and in the 'dead-zone' in $e^{+} e^{-}$processes can be enhanced by a factor of ALPFAC (default $=1$ ). The normalised electric charges of the fundamental fermions are stored in the array $\mathrm{QFCH}(\mathrm{I})$, where $\mathrm{I}=1-6$ for the quarks $d, u, s, c, b, t$ (e.g. $\mathrm{QFCH}(4)=2 . / 3$.) and 11-16 for the leptons $e, \nu_{e}, \mu, \nu_{\mu}, \tau, \nu_{\tau}$.

The weak neutral current is taken to be of the form $e\left(v_{f}+a_{f} \gamma_{5}\right) \gamma_{\mu}$, where the electric charge is evaluated at a scale appropriate to the process. The arrays $\mathrm{VFCH}(\mathrm{I}, \mathrm{J})$ and $\operatorname{AFCH}(I, J)$ store the couplings: $I$ as before, $J=1$ for the minimal Standard Model and 2 for possible $Z^{\prime}$ couplings (only used if ZPRIME=.TRUE.). Note that 
universality is not assumed - couplings can be arbitrarily set separately for each fermion species. The default couplings are given in terms of of $\operatorname{SWEIN}=\sin ^{2} \theta_{W}$, default value 0.2319 , as:

$$
v_{f}=\left(T_{3} / 2-Q \sin ^{2} \theta_{W}\right) /\left(\cos \theta_{W} \sin \theta_{W}\right), \quad a_{f}=T_{3} /\left(2 \cos \theta_{W} \sin \theta_{W}\right) .
$$

The weak charged current is given in terms of $g=e / \sin \theta_{W}$ and the CabbiboKobayashi-Maskawa mixing matrix, the elements squared of which are stored in $\operatorname{VCKM}(\mathrm{K}, \mathrm{L}), \mathrm{K}=1,2,3$ for $u, c, t, \mathrm{~L}=1,2,3$ for $d, s, b$. The variable $\mathrm{SCABI}=$

$\sin ^{2} \theta_{\text {Cabibbo }}$ is however also retained for the present. Note the Fermi constant $G_{\text {Fermi }}$ is eliminated from all cross sections.

The overall scale for all cross sections, given in nanobarns, is set by GEV2NB = $(\hbar c / e)^{2}$, default value 389379 .

We now give more detailed descriptions of the various subprocesses, concentrating again on the new features since ref. [i]1].

\subsection{Lepton-antilepton Standard Model processes}

Lepton beam polarisation effects are included in $e^{+} e^{-} \rightarrow 2 / 3$ jet production and the Bjorken process ( $Z H$ production). Incoming lepton and antilepton beam polarisations are specified by setting the two 3-vectors EPOLN and PPOLN: component 3 is longitudinal and 1,2 transverse.

Photon initial-state radiation (ISR) in $e^{+} e^{-}$annihilation events is allowed. The parameter TMNISR sets the minimum $\hat{s} / s$ value $\left(\right.$ default $=10^{-4}$ ), ZMXISR sets the (arbitrary) separation between unresolved and resolved emission (default $=1-10^{-6}$ ). Setting ZMXISR $=0$ switches off photon ISR.

\subsubsection{IPROC $=100-127$ : hadron production}

A correction to hard gluon emission in $e^{+} e^{-}$events has been added and is now the default process for IPROC $=100+\mathrm{IQ}$. The $\mathcal{O}\left(\alpha_{\mathrm{S}}\right)$ matrix element is used to add events in the 'dead zone' of phase-space corresponding to a quark-antiquark pair recoiling from a hard gluon [i] $[\overline{6}$. Although this is asymptotically negligible, and cannot be produced within the shower itself, it has a significant effect at LEP1 energies. As a result, the default parameters have been retuned, and show a marked improvement in agreement with $e^{+} e^{-}$data for event shapes sensitive to three-jet configurations.

The routine HWBDED implements this hard correction while HWBRAN has been modified to include the soft matrix-element corrections described in section

When IPROC $=100+I Q$, hard gluons emitted into the dead zone are assigned to the quark or antiquark shower and do not appear explicitly in the event record.

The $q \bar{q} g$ process alone, generated according to the $\mathcal{O}\left(\alpha_{\mathrm{S}}\right) q \bar{q} g$ matrix element with a maximum thrust cutoff THMAX (default 0.9 ), is given by IPROC $=110+$ IQ .

The uncorrected $q \bar{q}$ process has been retained for comparative purposes and is available as IPROC $=120+$ IQ. 
The fictional $e^{+} e^{-}$processes $e^{+} e^{-} \rightarrow g+g(+g)$, IPROC $=107$ and 127, is treated just like $e^{+} e^{-} \rightarrow q \bar{q}$, summed over light quark flavours, for direct comparisons between quark and gluon jets.

\subsubsection{IPROC $=150-250$ : lepton and electroweak boson production}

In IPROC $=150$, only the $s$-channel process, mediated by a virtual photon or $Z^{0}$, is included, so the final-state leptons must be different from the initial ones.

The processes of $W^{+} W^{-}$and $Z^{0} Z^{0}$ pair production, IPROC=200 and 250, are based on a program kindly supplied by Zoltan Kunszt, which fully includes decay correlations. The QCD $\mathcal{O}\left(\alpha_{\mathrm{S}}\right)$ matrix element correction for hard gluon emission in hadronic $W$ and $Z$ decays has also been implemented in these processes, according to the method described in section $3 . \overline{2} . \overline{3_{i}}$ In contrast to IPROC $=100+\mathrm{IQ}$, any hard gluons emitted into the dead zone are shown explicitly in the event record.

\subsubsection{IPROC $=300-499$ : Higgs boson production}

HERWIG generates SM Higgs bosons in lepton-antilepton collisions through the Bjorken process $Z^{(*)} \rightarrow Z^{(*)} H_{\mathrm{SM}}^{0}$ with one or both $Z^{0}$ 's off-shell $($ IPROC $=300+$ ID $)$ and $W^{+} W^{-} / Z^{0} Z^{0}$ fusion (IPROC $=400+$ ID). See section 's.'4 for explanation of how the Higgs decay is controlled by the value of ID.

\subsubsection{IPROC $=500-559$ : two-photon/photon-boson processes}

In the $e^{+} e^{-}$two-photon processes, IPROC $=500+\mathrm{ID}, \mathrm{ID}=0-10$ is the same as in Higgs processes for $q \bar{q}, \ell \bar{\ell}$ and $W^{+} W^{-}$. The Equivalent Photon Approximation (EPA) is used for the $e \rightarrow e \gamma$ vertices. The phase space is controlled by EMMIN and EMMAX for the two-photon centre-of-mass frame (CMF) mass, PTMIN and PTMAX for the transverse momentum of the CMF in the lab, and CTMAX for the c.m. angle of the outgoing particles. The additional phase-space variable WHMIN sets the minimum allowed hadronic mass and affects photoproduction reactions $(\gamma$-hadron and $\gamma-\gamma)$ and DIS.

In photon- $W^{ \pm}$fusion, IPROC $=550+$ ID, ID $=0-9$ is also the same as in Higgs processes, except that $\mathrm{ID}=1$ or 2 both give the sum of $d \bar{u}$ and $u \bar{d}$ etc. The EPA is used for the $e \rightarrow e \gamma$ vertex. The phase space is controlled by EMMIN and EMMAX only. The full $2 \rightarrow 3$ matrix elements for $\gamma e \rightarrow f \bar{f}^{\prime} \nu$ are used, so the cross section for real $W^{ \pm}$production is correctly included. In the case of $\gamma \gamma \rightarrow W W$ the decay correlations are not yet correctly included: the $W^{\prime}$ 's currently decay isotropically.

\subsubsection{IPROC $=600-656$ : four jet production}

Electron-positron annihilation to four jets is provided by IPROC $=600+I Q$, where a non-zero value for IQ guarantees production of quark flavour IQ whilst IQ $=0$ corresponds to the natural flavour mix. IPROC $=650+I Q$ is as above but without those terms in the matrix element which orient the event w.r.t. the lepton beam direction. 


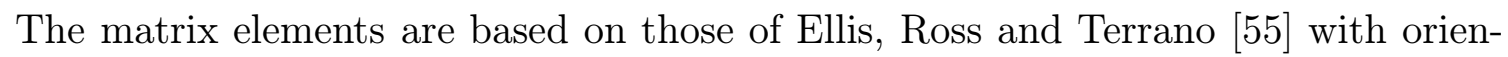
tation terms from Catani and Seymour $[\underline{\underline{5}} \overline{\underline{6}}]$. The soft and collinear divergences are avoided by imposing a minimum $y_{\text {cut }}$, Y4JT (default 0.01), on the initial four partons. The interjet distance $y_{\text {cut }}$ is calculated using either the Durham or JADE metrics, as selected by the logical variable DURHAM (default . TRUE.). In order to improve efficiency parameterizations of the volume of four-body phase space are used: these are accurate up to a few percent for $y_{\text {cut }}$ values less than 0.14 . Note also that the phase space is for massless partons, as are the matrix elements, though a mass threshold cut is applied.

The argument of the strong coupling is set equal to EMSCA, the scale for the parton showers. This is taken to be the smallest of the $y_{\text {cut }}$ values times the c.m. energy squared if FIX4JT=.FALSE. (default); otherwise the argument is fixed at Y4JT times the c.m. energy squared.

The matrix elements for the $q \bar{q} g g$ and $q \bar{q} q \bar{q}$ (same flavour quark) final states receive contributions from two colour flows each. The actual process and colour flow generated is indicated by IHPRO as shown in table ' $\overline{9}_{-}^{-}$. The meaning of 'c/f conn.' is discussed in section ${ }_{-1}^{1} . \overline{6} \cdot \overline{2}$ below. The treatment of the interference terms between the

\begin{tabular}{|c|c|c|}
\hline IHPRO & $\gamma^{\star} \rightarrow 1+2+3+4$ & c/f conn. \\
\hline 91 & $q+\bar{q}+g+g$ & 3142 \\
92 & $q+\bar{q}+g+g$ & 4123 \\
93 & $q+\bar{q}+q+\bar{q}$ & 4123 \\
94 & $q+\bar{q}+q+\bar{q}$ & 2143 \\
95 & $q+\bar{q}+q^{\prime}+\bar{q}^{\prime}$ & 4123 \\
\hline
\end{tabular}

Table 9: Four jet subprocesses. two colour flows is controlled by the array IOP4JT (1) for $q \bar{q} g g$ and IOP4JT (2) for $q \bar{q} q \bar{q}$ (identical quark flavour):

$$
\operatorname{IOP} 4 \mathrm{JT}(1)=\left\{\begin{array}{l}
0 \text { neglect } \\
1 \text { extreme } 3142 \\
2 \text { extreme } 4123
\end{array} \quad \text { IOP4JT(2) }=\left\{\begin{array}{l}
0 \text { neglect } \\
1 \text { extreme } 4123 \\
2 \text { extreme } 2143
\end{array}\right.\right.
$$

In both instances the default value is 0 .

See ref. [in for some applications and discussions of the new four-jet implementation in $e^{+} e^{-}$annihilations.

\subsection{Lepton-antilepton supersymmetric processes (MSSM)}

The R-parity conserving lepton-antilepton SUSY processes have IPROC $=700-799$ and $R_{\mathrm{p}}$ processes have IPROC $=800-899$. Lepton beam polarization effects are not included for any of the SUSY production processes. The processes have all been implemented in such a way as to allow either $e^{+} e^{-}$or $\mu^{+} \mu^{-}$as the initial state. As with the SM lepton-antilepton processes, ISR is allowed for the SUSY production processes.

As, by probing the individual thresholds, it may be possible to study the production of a given sparticle pair in lepton-antilepton collisions, we have provided more 
control over the sparticles produced than for the hadron-hadron SUSY production processes described in section $i_{-} \bar{A} . \overline{1}$.

We remind the reader here that all SUSY particle data have to be read from an input file before event generation (see section $1 . \overline{3} \cdot \overline{-} . \overline{1} \cdot \overline{1})$.

\subsubsection{IPROC $=700-799$ : gaugino, slepton and/or squark production}

With IPROC $=700$ one obtains the four processes IPROC $=710,730,740,760$ in the correct proportions. The matrix elements have been derived independently and the cross sections are in good agreement with those from SUSYGEN [5 these processes the hard process scale EMSCA has been set to the centre-of-mass energy.

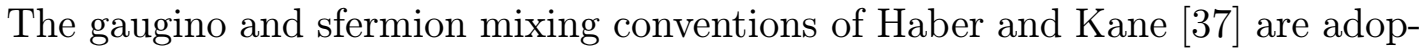
ted in all cases. In addition, the neutralino mixing matrix ZMIXSS is defined internally in terms of the photino, zino and current eigenstate neutral higgsino components, instead of the bino, $W_{3}$-ino and higgsino components adopted for ZMXNSS, equivalent

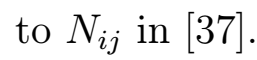

IPROC $=710$ gives lepton-antilepton $\rightarrow$ neutralino pair production. A number of additional IPROC codes have been provided to enable the user to produce given neutralino mass eigenstates. It should be noted that in order to provide a compact code for these processes there is more than one possible IPROC number for some processes. For example the final state $\widetilde{\chi}_{1}^{0} \widetilde{\chi}_{3}^{0}$ can be produced using either the IPROC codes 713 or 721 .

IPROC $=730$ gives lepton-antilepton $\rightarrow$ chargino pair production. As with the neutralino production there are codes to allow a given pair of charginos to be produced.

IPROC $=740$ gives lepton-antilepton $\rightarrow$ slepton pair production. In these processes for the first two generations the left/right eigenstates are produced, while for the third generation the mass eigenstates are produced. In the processes producing given slepton pairs for $\tilde{\tau}$ production processes the left eigenstate is replaced by the lighter mass eigenstate and the right eigenstate by the heavier mass eigenstate.

IPROC $=760$ gives lepton-antilepton $\rightarrow$ squark pair production. As with the other processes additional codes are provided to allow the production of given $\widetilde{q} \widetilde{q}^{*}$ pairs. For stop and sbottom production the mass eigenstates are produced, while for the first two generations the left/right eigenstates are generated. As with the slepton production for the third generation when a given squark pair is requested the left eigenstate is replaced by the lighter mass eigenstate and the right eigenstate by the heavier mass eigenstate. 


\subsection{Other lepton-antilepton non-Standard-Model processes}

\subsubsection{IPROC $=800-899:$ R-parity violating SUSY processes}

A range of possible $R_{\mathrm{p}}$ production processes in lepton-antilepton collisions is included. Unlike the case of $R_{p}$ production in hadron-hadron collisions, section $4.1 ;$ we have included processes for which there is either no $s$-channel resonance or the resonance is not kinematically accessible.

All the possible single sparticle production mechanisms which occur via the first term in the superpotential given in [3]9] are included. This includes the process $\ell^{+} \ell^{-} \rightarrow \gamma \widetilde{\nu}$ for which there is no $s$-channel resonance. As the cross section for this process diverges in the limit that the photon is collinear with the incoming lepton beams a cut on the $p_{T}$ of the outgoing particles $p_{T}>$ PTMIN has been imposed for this process $($ IPROC $=850)$. The ISR is switched off for this process as including it would lead to a double counting of the photon radiation. For this reason this process is not included in the code IPROC $=800$ which generates all the other single sparticle production mechanisms.

We have also included some processes for the production of SM particles via $s$-channel sneutrino exchange. In addition to the $s$-channel sneutrino diagrams the $t$ channel sparticle exchanges, SM diagrams and all the interference terms are included. This uses a generalisation of the formulae of [59]. A cut $p_{T}>$ PTMIN is used in the process $\ell^{+} \ell^{-} \rightarrow \ell^{+} \ell^{-}($IPROC $=870)$ to avoid the divergence as $t \rightarrow 0$ in the Bhabha scattering cross section.

Except where stated explicitly above, no PTMIN cut is applied.

\subsection{Hadron-hadron Standard Model processes}

\subsubsection{IPROC $=1300-1499$ : Drell-Yan processes}

The Drell-Yan code is extended to the production of all fermion pairs; 1300 gives all quark flavours; 1300+IQ a specific quark flavour, 1350 all leptons (including neutrinos) 1350+IL a specific lepton flavour. The $s$-channel component of the interference with like-flavour $q \bar{q}$ scattering is included here.

The initial-state parton showers in Drell-Yan processes are matched to the exact $\mathcal{O}\left(\alpha_{S}\right)$ matrix-element result as discussed in section 3.2 .3 The routine HWBDP implements the hard corrections whilst HWSBRN includes the soft corrections to the initial-state radiation. For further details see ref. [20 $\left.\overline{2}_{\overline{1}}\right]$.

\subsubsection{IPROC $=1500:$ QCD $2 \rightarrow 2$ processes}

At present only $2 \rightarrow 2$ subprocesses are implemented. They are classified in table 'i 10 . Here and in other subprocess tables, 'c/f conn.' refers to the colour/flavour connections between the partons: ' $i j k l$ ' means that the colour of parton 1 comes from parton $i$, that of 2 from $j$, etc. For antiquarks, which have no colour (only anticolour), the label shows instead to which parton the flavour is connected. For 


\begin{tabular}{|c|c|c|c|c|}
\hline IHPRO & $1+2$ & $\rightarrow$ & $3+4$ & $\mathrm{c} / \mathrm{f}$ conn. \\
\hline 1 & $q+q$ & $\rightarrow$ & $q+q$ & 3421 \\
\hline 2 & $q+q$ & $\rightarrow$ & $q+q$ & 4312 \\
\hline 3 & $q+q^{\prime}$ & $\rightarrow$ & $q+q^{\prime}$ & 3421 \\
\hline 4 & $q+\bar{q}$ & $\rightarrow$ & $q^{\prime}+\bar{q}^{\prime}$ & 2413 \\
\hline 5 & $q+\bar{q}$ & $\rightarrow$ & $q+\bar{q}$ & 3142 \\
\hline 6 & $q+\bar{q}$ & $\rightarrow$ & $q+\bar{q}$ & 2413 \\
\hline 7 & $q+\bar{q}$ & $\rightarrow$ & $g+g$ & 2413 \\
\hline 8 & $q+\bar{q}$ & $\rightarrow$ & $g+g$ & 2341 \\
\hline 9 & $q+\bar{q}^{\prime}$ & $\rightarrow$ & $q+\bar{q}^{\prime}$ & 3142 \\
\hline 10 & $q+g$ & $\rightarrow$ & $q+g$ & 3142 \\
\hline 11 & $q+g$ & $\rightarrow$ & $q+g$ & 3421 \\
\hline 12 & $\bar{q}+q$ & $\rightarrow$ & $\bar{q}^{\prime}+q^{\prime}$ & 3142 \\
\hline 13 & $\bar{q}+q$ & $\rightarrow$ & $\bar{q}+q$ & 2413 \\
\hline 14 & $\bar{q}+q$ & $\rightarrow$ & $\bar{q}+q$ & 3142 \\
\hline 15 & $\bar{q}+q$ & $\rightarrow$ & $g+g$ & 3142 \\
\hline 16 & $\bar{q}+q$ & $\rightarrow$ & $g+g$ & 4123 \\
\hline 17 & $\bar{q}+q^{\prime}$ & $\rightarrow$ & $\bar{q}+q^{\prime}$ & 2413 \\
\hline 18 & $\bar{q}+\bar{q}$ & $\rightarrow$ & $\bar{q}+\bar{q}$ & 4312 \\
\hline 19 & $\bar{q}+\bar{q}$ & $\rightarrow$ & $\bar{q}+\bar{q}$ & 3421 \\
\hline 20 & $\bar{q}+\bar{q}^{\prime}$ & $\rightarrow$ & $\bar{q}+\bar{q}^{\prime}$ & 4312 \\
\hline 21 & $\bar{q}+g$ & $\rightarrow$ & $\bar{q}+g$ & 2413 \\
\hline 22 & $\bar{q}+g$ & $\rightarrow$ & $\bar{q}+g$ & 4312 \\
\hline 23 & $g+q$ & $\rightarrow$ & $g+q$ & 2413 \\
\hline 24 & $g+q$ & $\rightarrow$ & $g+q$ & 3421 \\
\hline 25 & $g+\bar{q}$ & $\rightarrow$ & $g+\bar{q}$ & 3142 \\
\hline 26 & $g+\bar{q}$ & $\rightarrow$ & $g+\bar{q}$ & 4312 \\
\hline 27 & $g+g$ & $\rightarrow$ & $q+\bar{q}$ & 2413 \\
\hline 28 & $g+g$ & $\rightarrow$ & $q+\bar{q}$ & 4123 \\
\hline 29 & $g+g$ & $\rightarrow$ & $g+g$ & 4123 \\
\hline 30 & $g+g$ & $\rightarrow$ & $g+g$ & 4312 \\
\hline 31 & $g+g$ & $\rightarrow$ & $g+g$ & 2413 \\
\hline
\end{tabular}

Table 10: QCD subprocesses.

this colour/flavour labelling all partons are defined as outgoing. Thus, for example, process 10 has colour connections 3142 , corresponding to the colour flow diagram:

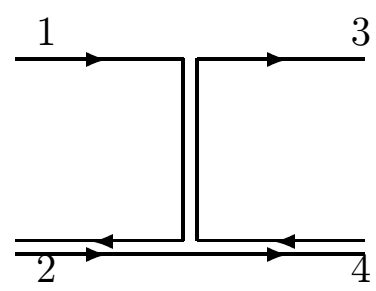


When different colour flows are possible, they are listed as separate subprocesses. This separation is not exact but is normally a good approximation [i6 ration is now performed using the improved method proposed in [29.1, as outlined in section is. 1 . The sum of the colour flows is the exact lowest-order cross section.

\subsubsection{IPROC $=1600-1699$ : Higgs boson production by parton fusion}

IPROC $=1600+$ ID gives the sum of $g g$ and $q \bar{q}$ fusion. The lowest-order formulae that we have used can be found in [3] mented in the subroutine HWHIGS.

\subsubsection{IPROC $=1700-1706$ : heavy quark production}

The separation of colour flows is now performed using the improved method proposed in $\left[\overline{2}_{2} \bar{g}\right]$, as outlined in section ' 15.1 .'. The classification of subprocesses according to IHPRO is as for IPROC $=1500$.

\subsubsection{IPROC=1800: QCD direct photon plus jet production}

The relevant IHPRO codes are 41-47 in table i1i: For future reference we also collect here the codes for other processes that involve outgoing direct

\begin{tabular}{|c|c|c|}
\hline IHPRO & $1+2 \rightarrow 3+4$ & c/f conn. \\
\hline 41 & $q+\bar{q} \rightarrow g+\gamma$ & 2314 \\
\hline 42 & $q+g \rightarrow q+\gamma$ & 3124 \\
\hline 43 & $\bar{q}+q \rightarrow g+\gamma$ & 3124 \\
\hline 44 & $\bar{q}+g \rightarrow \bar{q}+\gamma$ & 2314 \\
\hline 45 & $g+q \rightarrow q+\gamma$ & 2314 \\
\hline 46 & $g+\bar{q} \rightarrow \bar{q}+\gamma$ & 3124 \\
\hline 47 & $g+g \rightarrow g+\gamma$ & 2314 \\
\hline 51 & $\gamma+q \rightarrow g+q$ & 1423 \\
\hline 52 & $\gamma+\bar{q} \rightarrow g+\bar{q}$ & 1342 \\
\hline 53 & $\gamma+g \rightarrow q+\bar{q}$ & 1423 \\
\hline 61 & $q+\bar{q} \rightarrow \gamma+\gamma$ & 2134 \\
\hline 62 & $\bar{q}+q \rightarrow \gamma+\gamma$ & 2134 \\
\hline 63 & $g+g \rightarrow \gamma+\gamma$ & 2134 \\
\hline 71 & $\gamma+q \rightarrow M(S=0)+q^{\prime}$ & 1432 \\
\hline 72 & $\gamma+q \rightarrow M(S=1)_{L}+q^{\prime}$ & 1432 \\
\hline 73 & $\gamma+q \rightarrow M(S=1)_{T}+q^{\prime}$ & 1432 \\
\hline 74 & $\gamma+\bar{q} \rightarrow M(S=0)+\bar{q}^{\prime}$ & 1432 \\
\hline 75 & $\gamma+\bar{q} \rightarrow M(S=1)_{L}+\bar{q}^{\prime}$ & 1432 \\
\hline 76 & $\gamma+\bar{q} \rightarrow M(S=1)_{T}+\bar{q}^{\prime}$ & 1432 \\
\hline
\end{tabular}

Table 11: Direct photon subprocesses. photons or incoming pointlike photons $($ IPROC $=2200,5000-5520)$. Note that the photon is colour/flavour-connected to itself. In the cases IHPRO $=71-76, M$ represents an $L=0$ meson (see IPROC $=5500$ ).

\subsubsection{IPROC $=1900-1999$ : Higgs boson production by weak boson fusion}

The $q \bar{q} \rightarrow q^{\left({ }^{\prime}\right)} \bar{q}^{\left({ }^{\prime}\right)} V V \rightarrow q^{\left({ }^{\prime}\right)} \bar{q}^{\left({ }^{\prime}\right)} H_{\mathrm{SM}}^{0}$ subprocesses, for $V V=W^{+} W^{-}, Z^{0} Z^{0}$, summed over initial and final state quarks can be invoked by setting IPROC $=1900+$ ID, with ID used to identify the Higgs decay.

The formulae used are well known in the literature and can be found e.g. in [2] $\left.{ }_{2} \overline{1}\right]$. This process is administered by the subroutine HWHIGW, which also handles the similar cases initiated by $e^{+} e^{-}$and $e^{ \pm} p$ collisions. 


\subsubsection{IPROC $=2000-2008$ : single top production}

The process of single top quark production by $W$-boson exchange includes so far only those processes initiated by a $b$-quark (or antiquark) and a first- or second-generation quark or antiquark. Note that this requires $b$-quarks to be available in the parton distribution functions, which is not the case for the default Owens 1.1 set (NSTRU =5).

\subsubsection{IPROC $=2100-2170$ : electroweak boson plus jet production}

The electroweak boson decay correlations and width are now correctly included in these processes.

\subsection{9 $\mathrm{IPROC}=2200$ : direct photon pair production}

See section 4.6 .51 for IHPRO codes $(61-63)$.

\subsubsection{IPROC $=2300-2399$ : Higgs boson plus jet production}

High transverse momentum, scalar Higgs production, in association with a jet, is available as IPROC $=2300$, within the SM. Only the top quark is included in the loops with IAPHIG controlling the approximation used: IAPHIG $=0$ gives the zero top mass limit, 1 (default) the exact result, 2 the infinite top mass limit. The various subprocesses are illustrated in table $1 \overline{1} 2$ i.

Note that the Higgs boson is colour/flavour connected to itself.

The relevant routines HWHGJ1, HWHGJA/B/C/D, HWUCI2 and HWULI2 use (non-standard Fortran 77) DOUBLE COMPLEX variables which may not be accepted by some compilers and are called COMPLEX $* 16$ by others. Users can change to COMPLEX variables, but this involves a risk of rounding errors

\begin{tabular}{|c|c|c|c|c|}
\hline IHPRO & $1+2$ & $\rightarrow$ & $3+4$ & $\mathrm{c} / \mathrm{f}$ conn. \\
\hline 81 & $q+\bar{q}$ & $\rightarrow$ & $g+H_{\mathrm{SM}}^{0}$ & 23314 \\
\hline 82 & $q+g$ & $\rightarrow$ & $q+H_{\mathrm{SM}}^{0}$ & 3124 \\
\hline 83 & $\bar{q}+q$ & $\rightarrow$ & $g+H_{\mathrm{SM}}^{0}$ & 3124 \\
\hline 84 & $\bar{q}+g$ & $\rightarrow$ & $\bar{q}+H_{\mathrm{SM}}^{0}$ & 2314 \\
\hline 85 & $g+q$ & $\rightarrow$ & $q+H_{\mathrm{SM}}^{0}$ & 2314 \\
\hline 86 & $g+\bar{q}$ & $\rightarrow$ & $\bar{q}+H_{\mathrm{SM}}^{0}$ & 3124 \\
\hline 87 & $g+g$ & $\rightarrow$ & $g+H_{\mathrm{SM}}^{0}$ & 2314 \\
\hline
\end{tabular}

Table 12: Higgs plus jet subprocesses. spoiling numerical cancellations.

\subsubsection{IPROC $=2400-2450$ : colour singlet exchange}

IPROC $=2400$ : Two-to-two parton scattering via exchange of a colour singlet, MuellerTang pomeron [6-1]. The fixed $\alpha_{\mathrm{S}}$ and $\omega_{0}$ are given by ASFIXD (default 0.25) and OMEGAO (0.3) respectively.

IPROC $=2450$ : Photon exchange, for like-flavour $q \bar{q}$ pairs including the $t$-channel component of the interference with $q \bar{q} \rightarrow q \bar{q}$ via an $s$-channel photon or $Z^{0}$.

\subsubsection{IPROC $=2500-2599$ : Higgs boson plus top quark pair production}

The SM $2 \rightarrow 3$ Higgs production subprocesses of the type $g g \rightarrow t \bar{t} H_{\mathrm{SM}}^{0}$ and $q \bar{q} \rightarrow$ $t \bar{t} H_{\mathrm{SM}}^{0}$, for any flavour of initial state quarks $q$, are new to HERWIG version 6 and 
are handled by the subroutines HWHIGQ and HWH2QH. They are invoked by setting IPROC $=2500+$ ID (both $g g$ and $q \bar{q}$ ), with ID administering the Higgs decay channels as in IPROC $=300+$ ID. The initial state quark flavours $q$ are always summed over. Notice that, given the size of the Yukawa couplings of the Higgs boson to quarks, in practice only the associated production with top quarks is of phenomenological relevance in the SM. The matrix elements used in the implementation can be found in ref. [2] $\left[\begin{array}{l}1 \\ 1\end{array}\right]$. The treatment of the Higgs width here is as described in section 13.4.

\subsubsection{IPROC $=2600-2799$ : Higgs plus weak boson production}

The associated production of SM Higgs scalars with $W^{ \pm}$(IPROC=2600-2699) and $Z^{0}$ (IPROC=2700-2799) gauge bosons initiated by quark-antiquark fusion via the $2 \rightarrow 2$ processes $q \bar{q} \rightarrow W^{ \pm} H_{\mathrm{SM}}^{0}$ and $q \bar{q} \rightarrow Z^{0} H_{\mathrm{SM}}^{0}$ is now available. A summation is as usual intended on the incoming quarks. The formulae given in [i2 1] are used. The production processes are generated by the two new subroutines HWHIGV and HWH2VH whereas the Higgs decays are administered through the ID increment, as in IPROC $=300+$ ID. Again, the treatment of the Higgs boson width here is as discussed in section 's..4.'.

\subsection{Hadron-hadron supersymmetric processes (MSSM)}

The R-parity conserving SUSY processes occupy the IPROC entries of the series 3000 (sparticle processes) and 3300-3600 (Higgs boson production), while $R_{\mathrm{p}}$ processes have IPROC $=4000-4199$.

As with the lepton-antilepton SUSY processes the SUSY particle data must be read in from an input file before event generation as described in section

particular, unlike those of the SM Higgs boson $H_{\mathrm{SM}}^{0}$, the widths and decay modes of the SUSY Higgs bosons are not computed by HERWIG.

\subsubsection{IPROC $=3000-3030$ : sparton, gaugino and/or slepton production}

With IPROC $=3000$ one obtains the three following processes, IPROC $=3010,3020$, 3030 , in the correct proportions. The variable IHPRO gives the subprocess actually generated.

The matrix elements have been derived independently and the cross sections are in good agreement with those from ISAJET [3]

The hard process scale EMSCA has to be chosen globally for all sparton processes, e.g. for the argument of the QCD coupling. This is done using the kinematics appropriate to production of the lightest supersymmetric particle (LSP) and

$$
\text { EMSCA }=\sqrt{\frac{2 \hat{s} \hat{t}^{\prime} \hat{u}^{\prime}}{\hat{s}^{2}+\hat{t}^{\prime 2}+\hat{u}^{\prime 2}}} .
$$

with $\hat{t}^{\prime}=\hat{t}-m^{2}, \hat{u}^{\prime}=\hat{u}-m^{2}$ where $m$ is the LSP mass. 
IPROC $=3010$ gives 2-parton $\rightarrow$ 2-sparton processes. All QCD sparton, i.e. squark and gluino, pair production processes are implemented. The matrix elements and the scheme for separating different colour flow parts are as given in [2.

IPROC $=3020$ gives 2 -parton $\rightarrow 2$-gaugino or gaugino+sparton processes. All gaugino, i.e. chargino and neutralino, pair production processes and gaugino-sparton associated production processes are implemented.

The gaugino and sfermion mixing conventions of Haber and Kane [3in] are used

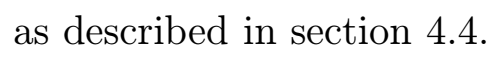

The various subprocesses, which include the $1 \leftrightarrow 2$ and charge conjugate reactions omitted below for brevity, are shown in table $1 \overline{1} \overline{-}$

Note that the gauginos connect to themselves. The indices $a, b, i, j$ label gauginos in the order of increasing mass and take values $a, b=1-2$ and $i, j=1-4$.

Gaugino mixing matrices are implemented in all subprocesses. The associated production subprocesses IHPRO $=3026,3027$ include stop and sbottom left-right mixings. CKM

\begin{tabular}{|c|ccl|c|}
\hline IHPRO & $1+2$ & $\rightarrow$ & $3+4$ & c/f conn. \\
\hline 3021 & $q+\bar{q}$ & $\rightarrow$ & $\widetilde{\chi}_{a}^{ \pm}+\widetilde{\chi}_{b}^{\mp}$ & 2134 \\
3022 & $q+\bar{q}$ & $\rightarrow$ & $\widetilde{\chi}_{i}^{0}+\widetilde{\chi}_{j}^{0}$ & 2134 \\
3023 & $q+\bar{q}^{\prime} \rightarrow$ & $\rightarrow$ & $\widetilde{\chi}_{a}^{ \pm}+\widetilde{\chi}_{i}^{0}$ & 2134 \\
3024 & $q+\bar{q}$ & $\rightarrow$ & $\widetilde{\chi}_{i}^{0}+\widetilde{g}$ & 2431 \\
3025 & $q+\bar{q}^{\prime}$ & $\rightarrow$ & $\widetilde{\chi}_{a}^{ \pm}+\widetilde{g}$ & 2431 \\
3026 & $g+q$ & $\rightarrow$ & $\widetilde{\chi}_{i}^{0}+\widetilde{q}$ & 2431 \\
3027 & $g+q$ & $\rightarrow$ & $\widetilde{\chi}_{a}^{ \pm}+\widetilde{q}$ & 2431 \\
\hline
\end{tabular}

Table 13: SUSY subprocesses. mixing is implemented in subprocesses IHPRO $=3023,3025,3027$ but neglected in subprocess IHPRO $=3021$.

IPROC $=3030$ gives 2-parton $\rightarrow 2$-slepton processes. All Drell-Yan slepton production processes are implemented. The formulae agree with those of refs. [6]_i, , 620 in the limit of no stau left-right mixing.

\subsubsection{IPROC $=3310-3375$ : Higgs-Higgs and Higgs-gauge boson pair production}

The production of Higgs-Higgs and Higgs-gauge boson pairs of the MSSM is implemented at tree level. We include gauge boson mediated contributions but not Higgstrahlung from the initial state, the only exception being $W^{ \pm} H^{\mp}$ production (IPROC $=3350)$, which does include diagrams where the Higgs boson couples to the initial partons as well as those mediated by neutral Higgs states [63]. Some of these processes are the MSSM equivalent of IPROC $=2600$ and 2700 described earlier for the case of the SM. Here, the cases IPROC $=3310(3320)$ correspond to $W^{ \pm} h^{0}\left(W^{ \pm} H^{0}\right)$ and IPROC $=3360(3370)$ to $Z^{0} h^{0}\left(Z^{0} H^{0}\right)$ final states. (No similar $A^{0}$ production can occur at leading order.) The array ENHANC is used to implement the MSSM couplings of Higgs scalars to gauge bosons.

N.B. The process code IPROC may get changed during generation of these processes. The original process code can be retrieved as IPROC+MAX (IMSSM, 0). 


\subsubsection{IPROC $=3410-3450$ : Higgs boson plus heavy quark production}

We have included so far only those processes initiated by a $b$-quark (or antiquark) and a gluon. Note that this requires $b$-quarks to be available in the parton distribution functions, which is not the case for the default Owens 1.1 set (NSTRU=5).

\subsubsection{IPROC $=3610-3630$ : neutral Higgs production by parton fusion}

These processes are the MSSM analogues of the SM processes 1600 etc. Recall however that the MSSM Higgs decay modes are controlled by the SUSY input data (see section $3 . \overline{1}$ ) and not by the value of IPROC. The subroutines HWHGS and HWHGT have been modified to take account of squark loop contributions and parity-violating Higgs-fermion couplings in the MSSM case.

N.B. The process code IPROC may get changed during generation of these processes. The original process code can be retrieved as IPROC+MAX (IMSSM, 0).

\subsection{Other hadron-hadron non-Standard-Model processes}

\subsubsection{IPROC $=4000-4199:$ R-parity violating SUSY processes}

We include all the possible production processes of resonant sleptons and squarks in hadron-hadron collisions, for arbitrary numbers of non-zero $R_{\mathrm{p}}$ couplings. These processes are implemented as two-to-two processes, i.e. with the decay of the resonant particle included. This allows us to include the $t$-channel diagrams where these occur. However we have not implemented those processes which can only occur via a $t$-channel diagram, or where the resonance will never be accessible. So for example while we include the process $u_{i} d_{j} \rightarrow \widetilde{b}_{1}^{*} Z^{0}$, which can occur via a resonant $\widetilde{b}_{2}^{*}$, we do not include the process $u_{i} d_{j} \rightarrow \widetilde{b}_{2}^{*} Z^{0}$, which cannot occur via a resonant diagram. In all cases both the processes listed and their charge conjugates are included.

The scale choice is $\sqrt{\hat{s}}$ rather than the conventional transverse mass, due to the large number of different processes which must be calculated. The colour connection

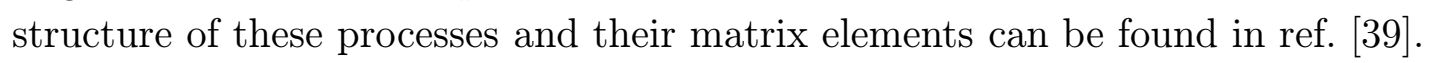

\subsubsection{IPROC $=4200-4299$ : graviton resonance production}

In some models with extra dimensions, Kaluza-Klein excitations of the graviton can be produced with significant cross sections at TeV-scale colliders. When the scale of the extra dimensions is not large, the excitations are manifest as discrete resonances.

The production of a resonant excitation of the graviton is implemented as a $2 \rightarrow 2$ process including the decay of the resonance. The process is treated in a model-independent way, assuming only that there is a universal coupling of the graviton resonance to the SM fields. The effective lagrangian is given by

$$
\mathcal{L}_{I}=-\frac{1}{\Lambda_{\pi}} h^{\mu \nu} T_{\mu \nu}
$$


where $h^{\mu \nu}$ is the spin-2 field and $T_{\mu \nu}$ is the energy-momentum tensor of the SM fields. Although in these models there are usually many resonances, we have implemented only one. Others can be studied by making appropriate changes in the parameters.

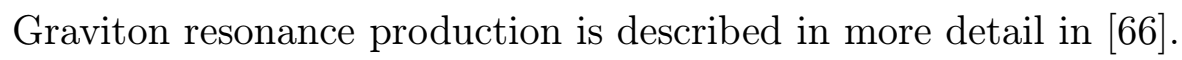

The process is controlled by the coupling GRVLAM $=\Lambda_{\pi}$, with dimensions of mass and default value $10 \mathrm{TeV}$, and by the mass EMGRV (default $1 \mathrm{TeV}$ ) and width GAMGRV of the resonance. If the width is set to zero (the default), the subroutine HWHGRV which calculates the cross section also calculates the width.

The parton-level cross section for this process is non-unitary and is proportional to $\hat{s} /$ EMGRV $^{4}$ at high energies. The fall-off of the parton distribution functions is not sufficient to suppress this bad high energy behaviour. Hence the parameters EMMIN and EMMAX controlling the minimum and maximum values of $\sqrt{\hat{s}}$ must be set. The default is to set these to $90 \%$ and $110 \%$ of the graviton resonance mass respectively. If the width of the resonance is more than a few percent of its mass then these limits should be reset.

After event generation, IHPRO is set to 50 for $q \bar{q}$ initiated subprocesses and to 51 for $g g$ initiated subprocesses.

\subsection{Photon-hadron and photon-photon processes}

\subsubsection{IPROC $=5000-5520:$ pointlike photon-hadron processes}

Pointlike photon-hadron scattering to produce QCD jets is available as IPROC = $5000-5206$. This is suitable for fixed-target photoproduction, provided events are generated in a frame in which the target has high momentum, and then boosted back to the lab. This is done if USECMF = .TRUE., the default, in which case the frame for event generation is the beam-target c.m. frame. IPROC $=5100+$ IQ gives flavour IQ pair production, $\gamma+g \rightarrow Q \bar{Q}$, and IPROC $=5200+$ IQ gives flavour IQ single excitation, $\gamma+Q \rightarrow g+Q$, both including quark masses. IPROC $=5000$ gives a sum over all processes and flavours, 5100 and 5200, with massless quark kinematics. In all cases, after event generation the code IHPRO is set to 51, 52 or 53 according to the hard subprocess, as specified in section 4.6 .5 . IPROC $=5300$ gives Compton scattering, $\gamma+q \rightarrow \gamma+q$.

The direct, higher twist, production of light $(u, d, s) \mathrm{L}=0$ mesons by point-like photons is also available: IPROC $=5500$ for all spin $=0$ and 1 mesons; 5510 for only $\mathrm{S}=0$ mesons; and 5520 for only $\mathrm{S}=1$ mesons. The vector mesons are produced with transverse or longitudinal polarisation and decayed accordingly. The corresponding IHPRO codes (71-76) are also listed in section '

All these processes are available with lepton as well as hadron beams, using the Equivalent Photon Approximation. The phase-space variable WHMIN sets the minimum allowed hadronic mass and affects photoproduction reactions ( $\gamma$-hadron and $\gamma-\gamma$ ) and DIS. In lepton-hadron DIS it is largely irrelevant since there is already 
a cut on Bjorken $y$ which at fixed $s$ is almost the same, but for lepton-gamma DIS it makes a big difference. Direct $\gamma+\gamma^{*} \rightarrow q+\bar{q}$ is included in the hard correction for lepton-gamma DIS.

\subsubsection{IPROC $=6000-6010:$ pointlike photon-photon processes}

Direct $\gamma \gamma \rightarrow$ charged particle pairs has been implemented with IPROC $=6000+$ IQ: if $\mathrm{IQ}=1-6$ then only quark flavour IQ is produced, if $\mathrm{IQ}=7,8$ or 9 then only lepton flavour $e, \mu$ or $\tau$ is produced and if $\mathrm{IQ}=10$ then only $W$ pairs are produced: in these cases particle mass effects are included. If IQ $=0$, the natural mix of quark pairs is produced using massless matrix elements but including a mass threshold cut. The range of allowed transverse momenta is controlled by PTMIN and PTMAX as usual.

\subsection{Baryon number violating processes}

\subsubsection{IPROC=7000-7999: generated by the HERBVI package}

An interface is provided to the HERBVI package for electroweak baryon number violation $(B)$, and other multi- $W^{ \pm}$production processes [i] $\left.\overline{1}_{-1}\right]$. For full details, see http ///WWW-thphys.physics.ox.ac.uk/users/PeterRichardson/HERWIG/herbvi/i

\subsection{Minimum bias soft hadron-hadron collisions}

\subsubsection{IPROC $=8000$ : Minimum bias soft hadron-hadron event}

Non-diffractive, soft hadronic, minimum bias events (IPROC $=8000)$ can be generated for the following combinations of beam and target: $p, \bar{p}, \pi^{ \pm}, K^{ \pm}, e^{ \pm}, \mu^{ \pm}, \gamma$ on target $p$ (or vice versa); $p, \bar{p}$ on target $n$ (or vice versa); or $\gamma$ on $\gamma$. The event weight is the estimated cross section based on the parameterizations of Donnachie and Land-

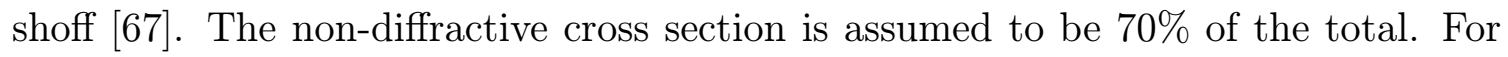
lepton beams a photon is first generated using the Effective Photon Approximation

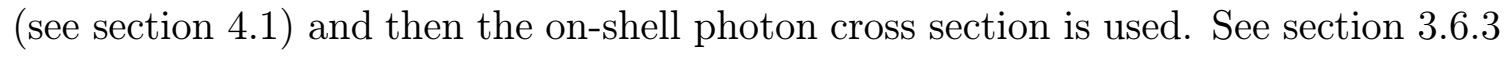
for discussion of the model used and the relevant parameters.

\subsection{Deep inelastic lepton scattering}

Deep inelastic (DIS) processes are broadly divided into those that start at $\mathcal{O}\left(\alpha_{\mathrm{S}}^{0}\right)$ $\left(\right.$ IPROC $\left.=90^{* *}\right)$ and those like heavy quark and dijet production which start at $\mathcal{O}\left(\alpha_{\mathrm{S}}^{1}\right)$ $\left(\right.$ IPROC $\left.=91^{* *}\right)$. Note that the DIS $\mathcal{O}\left(\alpha_{\mathrm{S}}\right)$ jet production processes, IPROC $=92^{* *}$, have been withdrawn since they are subsumed within IPROC $=91^{* *}$.

The default limits on $Q^{2}$ in DIS processes (Q2MIN, Q2MAX) have been set very small/large $\left(0,10^{10} \mathrm{GeV}\right)$ and are reset to the kinematic limits unless changed by the user. This means the default Q2MIN is not suitable for simple neutral current DIS (IPROC $=9000$ etc), but is appropriate for jet and heavy quark photoproduction. The range of the Bjorken- $y$ variable $\left(y=Q^{2} / x s\right)$ can be limited by YBMIN and YBMAX. 
The kinematic reconstruction of DIS processes can now take place in the Breit frame, if BREIT =.TRUE. (the default value). Previous versions used the lab frame. Although the reconstruction is fully invariant under Lorentz boosts along the incoming hadron's direction, it is not under transverse boosts, so there should be some difference between the two frames. The boost is not performed for very small $Q^{2}\left(<10^{-4}\right)$ to avoid numerical instabilities, but the two frames are in any case equivalent for such small values.

The phase space for boson-gluon fusion is controlled by the parameters EMMIN, EMMAX (see section $1 \overline{3} \cdot \overline{3} \cdot \overline{4}$ ). The default values $(0, \sqrt{s})$ correspond to the behaviour of version 5.1 .

Lepton beam polarisation effects are included in all DIS processes apart from $J / \psi$ production. The polarisation is specified as in lepton-antilepton processes, i.e. by setting the 3 -vectors EPOLN and PPOLN: component 3 is longitudinal and 1,2 transverse. Transverse only occurs in $e^{+} e^{-}$routines; recall that two transverse 'measurements' are needed to see an effect so it should not arise elsewhere. Note that in DIS processes one has to set either EPOLN if it is a lepton or (exclusive) PPOLN if an antilepton.

All the DIS processes IPROC $=9000-9599$ are available in $e^{+} e^{-}$as well as leptonhadron collisions. The program generates a photon from the second beam (only) in the Equivalent Photon Approximation and the default is to use Drees-Grassie [4] structure functions for DIS on the photon.

The parameter WHMIN sets the minimum allowed hadronic mass in DIS. In leptonhadron DIS it is largely irrelevant since there is already a cut on Bjorken $y$ which at fixed $s$ is almost the same but for lepton-gamma DIS it makes a big difference.

In addition to the processes listed here, a full simulation of QCD instanton-in-

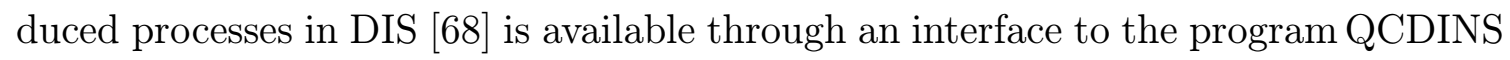

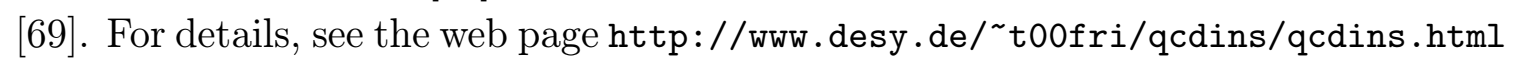

\subsubsection{IPROC $=9000-9006:$ neutral current}

Matrix-element corrections to DIS are available, following the general method described in section correction is included in routines HWBRAN and HWSBRN for the final- and initial-state radiation respectively.

\subsubsection{IPROC $=9010-9016:$ charged current}

These are the charged current processes corresponding to those above, with the same treatment of hard and soft matrix element corrections.

\subsubsection{IPROC $=9100-9130: \mathcal{O}\left(\alpha_{\mathrm{S}}\right)$ neutral current processes}

The photoproduction processes have been extended from the original heavy quark production program, to include all quark pair production (IPROC=9100-9106) and QCD Compton (IPROC $=9110-9122)$, as well as the sum of the two (IPROC=9130). 
The possible flavours for processes 9100,9110 and 9130 are limited by the input parameters IFLMIN and IFLMAX (defaults are 1 and 3, i.e. only $u, d, s$ flavours).

A sign error has been corrected that led to the incorrect sign for the lepton-jet azimuthal correlation in QCD Compton processes in versions before 5.7.

$J / \psi$ production $($ IPROC $=9107)$ now uses the Equivalent Photon Approximation instead of Weizsacker-Williams, with the same phase-space cuts as hadronic processes

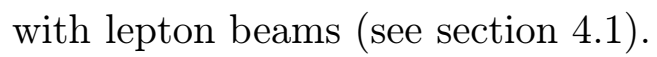

The argument of the running coupling is controlled by the parameter BGSHAT see section ${ }^{1}{ }_{-1}^{\prime}$.

\subsubsection{IPROC $=9140-9144$ : charged-current heavy quark production}

At present only $W^{ \pm} g$ fusion processes are implemented.

\subsubsection{IPROC $=9500-9599$ : Higgs boson production by weak boson fusion}

The process of $W^{+} W^{-} / Z^{0} Z^{0}$ fusion into the SM Higgs boson is also available in $e^{ \pm} p$ collisions, as IPROC $=9500+$ ID. For details of the implementation, see section 'B..4t' and the corresponding processes initiated by lepton-lepton and hadron-hadron collisions, IPROC $=400+$ ID and IPROC $=1900+$ ID, respectively.

\subsection{Including new subprocesses}

The procedure for including further subprocesses remains substantially as described in ref. [1] but is repeated here for completeness.

The parton and hard subprocess 4-momenta, masses and identity codes need to be entered in COMMON/HEPEVT/ with the appropriate status codes ISTHEP(I) = 110-114 to tell the program which is which (see the table in section $\left.18 . \overline{3} \cdot \overline{1}_{1}\right)$. The colour/flavour structure should be specified by the second mother and daughter pointers as explained in section $1 \overline{4} .6 .2$.

The HERWIG identity codes IDHW(I) in COMMON/HWEVNT/ also need to be set correctly. The IDHW codes can be listed in a run with IPRINT $=2$ : the most important are the quarks 1-6 (as IDHEP), antiquarks 7-12, gluon 13, overall centre-of-mass 14, hard centre-of-mass 15, soft centre-of-mass 16, photon 59, leptons 121-126, antileptons $127-132$.

The utility subroutine HWUIDT (IOPT, IPDG, IHWG, NAME) is provided to translate between Particle Data Group code IPDG, HERWIG code IHWG, and HERWIG CHARACTER $* 8$ NAME, with IOPT $=1,2,3$ depending on which of IPDG, IHWG and NAME is the input argument.

Consider for example the process of virtual photon-gluon fusion to make $b+\bar{b}$ in proton-electron collisions (in fact this process is included as IPROC $=9105$ ). We assume the user provides a subroutine to generate the momenta PHEP for the hard 


\begin{tabular}{|c|c|c|c|c|c|c|}
\hline IHEP & Entry & ISTHEP & IDHEP & JMOHEP & JDAHEP & IDHW \\
\hline 1 & $e$ beam & 101 & 11 & 00 & 00 & 121 \\
2 & $p$ beam & 102 & 2212 & 00 & 00 & 73 \\
3 & $e p$ c.m. & 103 & 0 & 00 & 00 & 14 \\
\hline 4 & $e$ in & 111 & 11 & 67 & 07 & 121 \\
5 & gluon & 112 & 21 & 69 & 08 & 13 \\
6 & hard cm & 110 & 0 & 45 & 79 & 15 \\
7 & $e$ out & 113 & 11 & 64 & 04 & 121 \\
8 & $b$ & 114 & 5 & 65 & 09 & 5 \\
9 & $\bar{b}$ & 114 & -5 & 68 & 05 & 11 \\
\hline
\end{tabular}

Table 14: Event record entries for $e g \rightarrow e b \bar{b}$.

subprocess $e+g \rightarrow e b \bar{b}$. The colour structure is

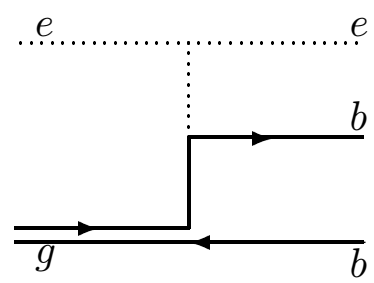

Thus the momenta generated, together with those of the initial beams and the overall centre of mass, could be entered in the sequence shown in talbe $1 \bar{i}_{-i}$.

Note that if there are more than two outgoing partons, the first has status 113 and all the others 114. Each parton has $\operatorname{JMOHEP}(1, \mathrm{I})=6$ to indicate the location of the hard c.m. for this subprocess, while $\operatorname{JMOHEP}(2, I)$ gives the location of the colour mother (treating the incoming gluon as outgoing) or the connected electron. $\operatorname{JDAHEP}(1, I)$ will be set by the jet generator $\operatorname{HWBGEN}$, while $\operatorname{JDAHEP}(2, I)$ points to the anticolour mother (or connected electron). Finally the HERWIG identifiers IDHW(I) could be set to the indicated values by means of the translation subroutine HWUIDT as follows:

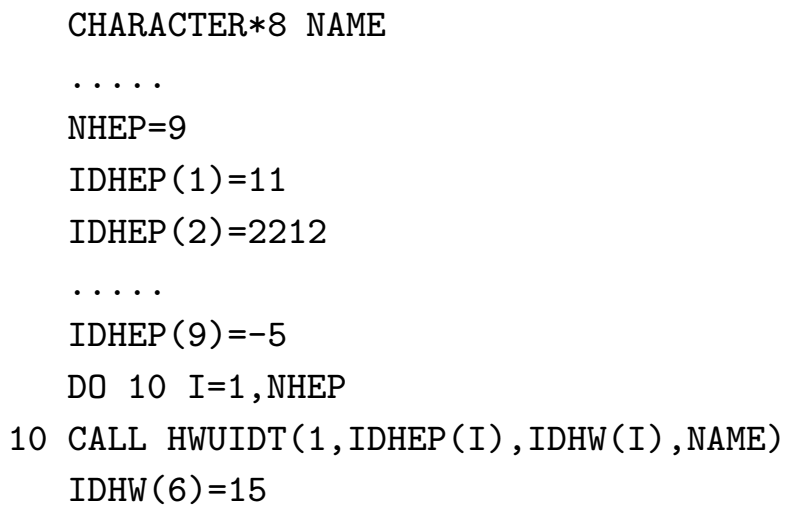

The last statement is needed because IDPDG(I) $=0$ returns IDHW(I) $=14$. If subroutine HWBGEN is now called, it will find the coloured partons and generate QCD jets from them. Subsequent calls to HWCFOR etc. can then be used to form clusters and hadronize them. 
If the hard subprocess routine is called from HWEPRO, like those already provided, it must have two options controlled by the logical variable GENEV in COMMON/HWHARD/. For GENEV = .FALSE., an event weight (normally the cross section in nanobarns) is generated and stored as EVWGT in COMMON/HWEVNT/. If this weight is accepted by HWEPRO, the subroutine is called a second time with GENEV $=$.TRUE. and the corresponding event data should then be generated and stored as explained above. On certain computers it will be necessary to SAVE those variables that determine event characteristics between the two subroutine calls.

The parameter NMXJET sets the maximum number of outgoing partons in a hard subprocess (default 200).

\section{Parameters}

The quantities that may be regarded as adjustable parameters are indicated in table 1.5 .5 . Notes on parameters are given below.

\begin{tabular}{|c|l|c|}
\hline Name & Description & Default \\
\hline QCDLAM & $\Lambda_{Q C D}$ (see below) & 0.18 \\
\hline RMASS $(1)$ & Down quark mass & 0.32 \\
RMASS(2) & Up quark mass & 0.32 \\
RMASS(3) & Strange quark mass & 0.50 \\
RMASS(4) & Charmed quark mass & 1.55 \\
RMASS(5) & Bottom quark mass & 4.95 \\
RMASS(6) & Top quark mass & 174.3 \\
\hline RMASS(13) & Gluon effective mass & 0.75 \\
\hline VQCUT & Quark virtuality cutoff (added to & 0.48 \\
& quark masses in parton showers) & \\
VGCUT & Gluon virtuality cutoff (added to & 0.10 \\
& effective mass in parton showers) & \\
VPCUT & Photon virtuality cutoff & 0.40 \\
\hline CLMAX & Maximum cluster mass parameter & 3.35 \\
CLPOW & Power in maximum cluster mass & 2.00 \\
PSPLT(1) & Split cluster spectrum parameter & 1.00 \\
PSPLT (2) & 1: light cluster, 2 heavy b-cluster & PSPLT(1) \\
\hline QDIQK & Maximum scale for gluon $\rightarrow$ diquarks & 0.00 \\
PDIQK & Gluon $\rightarrow$ diquarks rate parameter & 5.00 \\
\hline QSPAC & Cutoff for spacelike evolution & 2.50 \\
PTRMS & Intrinsic $p_{T}$ in incoming hadrons & 0.00 \\
\hline
\end{tabular}

Table 15: Adjustable parameters. 
- QCDLAM can be identified at high momentum fractions ( $x$ or $z$ ) with the fundamental 5-flavour QCD scale $\Lambda \frac{(5)}{\mathrm{MS}}$. However, this relation does not necessarily hold in other regions of phase space, since higher order corrections are not treated precisely enough to remove renormalization scheme ambiguities [i]

- $\operatorname{RMASS}(1,2,3,13)$ are effective light quark and gluon masses used in the hadronization phase of the program. They can be set to zero provided the parton shower cutoffs VQCUT and VGCUT are large enough to prevent divergences (see below).

- For cluster hadronization, it must be possible to split gluons into $q \bar{q}$, i.e. RMASS(13) must be at least twice the lightest quark mass. Similarly it may be impossible for heavy-flavoured clusters to decay if $\operatorname{RMASS}(4,5)$ are too low.

- VQCUT and VGCUT are needed if the quark and gluon effective masses become small. The condition to avoid divergences in parton showers is

$$
\frac{1}{Q_{i}}+\frac{1}{Q_{j}}<\frac{1}{\text { QCDL3 }}
$$

for either $i$ or $j$ or both gluons, where $Q_{i}=\operatorname{RMASS}(i)+\operatorname{VQCUT}$ for quarks, RMASS(13) + VGCUT for gluons, and QCDL3 is the three-flavour QCD scale used internally by HERWIG. QCDL3 is obtained by matching at the $b$ - and $c$-quark mass scales from the internal five-flavour scale

$$
\text { QCDL5 }=\text { QCDLAM } \times \exp \left(\frac{151-9 \pi^{2}}{138}\right) / \sqrt{2}=1.109 \times \text { QCDLAM } .
$$

Note that, in the notation of ref. [1] 3 in and section 3.2, QCDL5 $=\Lambda_{\text {phys }} / \sqrt{2}$ for five flavours.

- VPCUT is the analogous quantity for photon emission. It now defaults to $0.4 \mathrm{GeV}$. Previous versions defaulted to $\sqrt{s}$, switching off such emission. Results after experimental cuts are insensitive to its exact value in the range 0.1 to $1.0 \mathrm{GeV}$.

- CLMAX and CLPOW determine the maximum allowed mass of a cluster made from quarks $i$ and $j$ as follows

$$
M^{\text {CLPOW }}<\operatorname{CLMAX}^{\text {CLPOW }}+(\operatorname{RMASS}(i)+\operatorname{RMASS}(j))^{\text {CLPOW }} .
$$

Since the cluster mass spectrum falls rapidly at high mass, results become insensitive to CLMAX and CLPOW at large values of CLMAX. Smaller values of CLPOW will increase the yield of heavier clusters (and hence of baryons) for heavy quarks, without affecting light quarks much. For example, the default value gives no $b$-baryons whereas CLPOW $=1.0$ makes $b$-baryons $/ b$-hadrons about $1 / 4$. 
- PSPLT determines the mass distribution in the cluster splitting $C \ell_{1} \rightarrow C \ell_{2}+C \ell_{3}$ when $C \ell_{1}$ is above the maximum allowed mass. The masses of $C \ell_{2}$ and $C \ell_{3}$ are generated uniformly in $M^{\text {PSPLT }}$. As long as the number of split clusters is small, dependence on PSPLT is weak.

- QDIQK greater than twice the lightest diquark mass enables non-perturbative gluon splitting into diquarks as well as quarks. The probability of this is PDIQK $\times d Q / Q$ for scales $Q$ below QDIQK. The diquark masses are taken to be the sum of constituent quark masses. Thus the default value QDIQK $=0$ suppresses gluon $\rightarrow$ diquark splitting.

- QSPAC is the scale below which the structure functions of incoming hadrons are frozen and non-valence constituent partons are forced to evolve to valence partons, if ISPAC $=0$. For ISPAC $=2$, structure functions are frozen at scale QSPAC, but evolution continues down to the infrared cutoff.

- PTRMS is the width of the (gaussian) intrinsic transverse momentum distribution of valence partons in incoming hadrons at scale QSPAC.

In practice, the parameters that have been found most effective in fitting data are QCDLAM, the gluon effective mass RMASS(13), and the cluster mass parameter CLMAX. Note that QSPAC, PTRMS and ENSOF do not affect lepton-lepton collisions.

The default parameter values are based on those that were found to give good agreement when comparing earlier versions with event shape distributions at LEP. However, the substantial changes in this version mean that a re-tuning of parameters would be very worthwhile.

Up-to-date details of HERWIG parameter tunes can be found via the official web page cited in section

\section{Control switches, constants and options}

A number of quantities can be reset to control the program and various options:

\begin{tabular}{|c|l|c|}
\hline Name & Description & Default \\
\hline NEVHEP & Current number of events & 0 \\
NHEP & Current number of entries in /HEPEVT/ & 0 \\
\hline IPRINT & Information to include in print out & 1 \\
MAXPR & Number of events to print out & 1 \\
PRVTX & Include vertex information in print out & .TRUE. \\
NPRFMT & Controls number of sig. figs. in print out & 1 \\
PRNDEC & Use decimal/hexadecimal in print out & .TRUE. \\
PRNDEF & Produce ASCII (stout) version of print out & .TRUE. \\
PRNTEX & Produce ITEX version of print out
\end{tabular}




\begin{tabular}{|c|c|c|}
\hline Name & Description & Default \\
\hline PRNWEB & Produce html version of print out & .FALSE. \\
\hline MAXER & Maximum number of errors to tolerate & 10 \\
\hline LWEVT & Unit for writing output events & 0 \\
\hline LRSUD & Unit for reading Sudakov table & 0 \\
\hline LWSUD & Unit for writing Sudakov table & 77 \\
\hline SUDORD & $\alpha_{\mathrm{S}}$ order in Sudakov table & 1 \\
\hline INTER & Order of interpolation in Sudakov tables & 3 \\
\hline $\operatorname{NRN}(1)$ & Random number seed 1 & 17673 \\
\hline $\operatorname{NRN}(2)$ & Random number seed 2 & 63565 \\
\hline WGTMAX & Max. weight (0 to search for it) & 0.0 \\
\hline NOWGT & Generate unweighted events with & \\
\hline & EVWGT $=$ AVWGT & . TRUE. \\
\hline AVWGT & Mean event weight & 1.0 \\
\hline EFFMIN & Min. acceptable Monte Carlo efficiency & 0.001 \\
\hline AZSOFT & Include soft gluon azimuthal correlations & TRUE. \\
\hline AZSPIN & Include gluon spin azimuthal correlations & . TRUE. \\
\hline HARDME & Use hard matrix-element corrections & . TRUE. \\
\hline SOFTME & Use soft matrix-element corrections & . TRUE. \\
\hline GCUTME & Gluon energy cut in top M.E. correction & 2.0 \\
\hline NCOLO & Number of colours & 3 \\
\hline NFLAV & Number of (producible) flavours & 6 \\
\hline $\operatorname{MODPDF}(\mathrm{I})$ & PDFLIB parton set and author group for beam & -1 \\
\hline $\operatorname{AUTPDF}(\mathrm{I})$ & I $(=1,2)$ (if MODPDF $<0$ do not use PDFLIB) & 'MRS' \\
\hline NSTRU & $\begin{array}{l}\text { Input parton set }(1,2=\text { Duke-Owens sets } 1,2 \\
3,4=\text { EHLQ } \text { sets } 1,2 ; 5=\text { Owens set } 1.1)\end{array}$ & 5 \\
\hline PRSOF & Probability of soft underlying event & 1.0 \\
\hline ENSOF & $\begin{array}{l}\text { Multiplicity enhancement for SUE: } \\
\qquad n=\left\langle n_{p \bar{p}}\right\rangle(\operatorname{ENSOF} \sqrt{s})\end{array}$ & 1.0 \\
\hline PMBN1 & Mean multiplicity in SUE/Min. bias event & +9.110 \\
\hline PMBN2 & $\left\langle n_{p \bar{p}}\right\rangle(\sqrt{s})=\mathrm{PMBN} 1 s^{\mathrm{PMBN} 2}+\mathrm{PMBN} 3$ & +0.115 \\
\hline PMBN3 & & -9.500 \\
\hline PMBK1 & Negative binomial param. & \\
\hline & $k^{-1}=$ PMBK1 $\log _{e}(s)+$ PMBK2 & +0.029 \\
\hline PMBK2 & & -0.014 \\
\hline PMBM1 & $\begin{array}{l}\text { Soft cluster mass spectrum: } \\
\qquad\left(M-M_{1}-M_{2}-\text { PMBM1 }\right) e^{- \text {PMBM2M }}\end{array}$ & 0.2 \\
\hline PMBM2 & & 2.0 \\
\hline
\end{tabular}




\begin{tabular}{|c|c|c|}
\hline Name & Description & Default \\
\hline PMBP1 & \multicolumn{2}{|l|}{ Soft cluster $P_{T}$ spectrum: $p_{T} e^{-\mathrm{PMBPi} \sqrt{p_{T}^{2}+M^{2}}}$} \\
\hline PMBP2 & $s, c$ quarks & 3.0 \\
\hline PMBP3 & diquarks & 5.2 \\
\hline IOPREM & Options for treatment of remnant clusters & 1 \\
\hline BTCLM & Mass parameter in remnant fragmentation & 1.0 \\
\hline VMIN2 & Min. parton virtuality ${ }^{2}$ in distance calcs. & 0.1 \\
\hline CLRECO & Include colour rearrangement & .FALSE. \\
\hline PRECO & Probability for rearrangement & $1 / 9$ \\
\hline EXAG & Lifetime scaling for weak bosons & 1.0 \\
\hline ETAMIX & mixing angle in degrees & -23 \\
\hline PHIMIX & mixing angle in degrees & +36 \\
\hline H1MIX & mixing angle in degrees & $\tan ^{-1}(1 / \sqrt{2})$ \\
\hline FOMIX & mixing angle in degrees & $\tan ^{-1}(1 / \sqrt{2})$ \\
\hline F1MIX & mixing angle in degrees & $\tan ^{-1}(1 / \sqrt{2})$ \\
\hline F2MIX & mixing angle in degrees & +26 \\
\hline ET2MIX & mixing angle in degrees & $\tan ^{-1}(1 / \sqrt{2})$ \\
\hline OMHMIX & mixing angle in degrees & $\tan ^{-1}(1 / \sqrt{2})$ \\
\hline PHЗMIX & mixing angle in degrees & +28 \\
\hline B1LIM & B cluster $\rightarrow 1$ hadron parameter & 0.0 \\
\hline $\operatorname{CLDIR}(\mathrm{I})$ & $\begin{array}{l}\text { Decay orientation of perturbative clusters, } \\
0 \text { : isotropic, } 1 \text { : along quark direction }\end{array}$ & 1 \\
\hline \multirow[t]{2}{*}{$\operatorname{CLSMR}(\mathrm{I})$} & Width of gaussian angle smearing, & 0.0 \\
\hline & $\mathrm{I}=1$ : light cluster, $\mathrm{I}=2$ : heavy $b$-cluster & $\begin{array}{l}\text { CL . . (1) }= \\
\text { CL . . (2) }\end{array}$ \\
\hline PWT (I) & $\begin{array}{l}\text { A priori weights for } f \bar{f} \text {-pairs in cluster decay, } \\
\mathrm{I}=1-6: f=d, u, s, c, b, t \mathrm{I}=7: f=q q^{\prime}\end{array}$ & 1.0 \\
\hline $\operatorname{REPWT}(L, J, N)$ & A priori weight for $n^{(2 S+1)} L_{J}$ mesons & 1.0 \\
\hline SNGWT & A priori weight for singlet baryons & 1.0 \\
\hline DECWT & A priori weight for decuplet baryons & 1.0 \\
\hline PLTCUT & Minimum lifetime for particle to be set stable & $1.0 \times 10^{-8}$ \\
\hline VTOCDK (I) & \multirow{2}{*}{$\begin{array}{l}\text { Veto decay of clusters to hadron } \mathrm{I} \\
\text { Veto decay of resonances to hadron I } \\
\mathrm{I}=290-293, f_{0}(980), a_{0}(980)\end{array}$} & .FALSE. \\
\hline $\operatorname{VTOCDK}(I)$ & & $\begin{array}{l}. \text { FALSE. } \\
\text {.TRUE. }\end{array}$ \\
\hline PIPSMR & \multirow{3}{*}{$\begin{array}{l}\text { Smear the primary vertex } \\
x \text { width }(\mathrm{mm}) \\
y \text { width }(\mathrm{mm})\end{array}$} & .FALSE. \\
\hline $\operatorname{VIPWID}(1)$ & & 0.25 \\
\hline $\operatorname{VIPWID}(2)$ & & 0.015 \\
\hline
\end{tabular}

Table 1i ${ }^{\text {i: }}$ Control switches, constants and options. (Continues) 


\begin{tabular}{|c|c|c|}
\hline Name & Description & Default \\
\hline $\operatorname{VIPWID}(3)$ & $z$ width $(\mathrm{mm})$ & 1.8 \\
\hline MAXDKL & Veto decays outside given volume & . FALSE. \\
\hline IOPDKL & Option for volume: $1=$ cylinder, $2=$ sphere & 1 \\
\hline DXRCYL & Radius for cylindrical option (mm) & 20 \\
\hline DXZMAX & Length for cylindrical option (mm) & 500 \\
\hline DXRSPH & Radius for spherical option (mm) & 100 \\
\hline BDECAY & $\begin{array}{l}\text { Controls which B Decay package is usd. The } \\
\text { allowed values are: 'HERW', 'EURO' or 'CLEO' }\end{array}$ & 'HERW' \\
\hline MIXING & Include neutral B meson mixing & . TRUE. \\
\hline $\operatorname{XMIX}(1)$ & $\Delta M / \Gamma$ for $B_{s}^{0}$ & 10.0 \\
\hline $\operatorname{XMIX}(2)$ & $\Delta M / \Gamma$ for $B_{d}^{0}$ & 0.7 \\
\hline $\operatorname{YMIX}(1)$ & $\Delta \Gamma / 2 \Gamma$ for $B_{s}^{0}$ & 0.2 \\
\hline $\operatorname{YMIX}(2)$ & $\Delta \Gamma / 2 \Gamma$ for $B_{d}^{0}$ & 0.0 \\
\hline RMASS (198) & $W^{+}$mass & 80.42 \\
\hline RMASS (199) & $W^{-}$mass & RMASS (198) \\
\hline GAMW & $W^{ \pm}$width & 2.12 \\
\hline RMASS (200) & $Z^{0}$ mass & 91.188 \\
\hline GAMZ & $Z^{0}$ width & 2.495 \\
\hline WZRFR & Use $W / Z$ rest frame for decay parton showers & . TRUE. \\
\hline $\operatorname{MODBOS}(I)$ & $\begin{array}{l}\text { Force decay modes for weak bosons, } \\
\text { see sect. }\end{array}$ & 0 \\
\hline RMASS (201) & SM Higgs mass & 115 \\
\hline IOPHIG & Options for large Higgs mass distribution & 3 \\
\hline GAMMAX & Limit on range of Higgs mass distribution & 10. \\
\hline \multirow[t]{2}{*}{$\operatorname{ENHANC}(\mathrm{I})$} & Enhancement factor for SM Higgs decay & \\
\hline & mode $I$ & 1.0 \\
\hline RMASS (209) & $\begin{array}{r}\text { Hypothetical 4th generation 'bottom' } \\
\text { quark mass }\end{array}$ & 200. \\
\hline RMASS (215) & $\begin{array}{r}\begin{array}{r}\text { Hypothetical 4th generation 'bottom' } \\
\text { antiquark mass }\end{array} \\
\end{array}$ & RMASS (209) \\
\hline ALPHEM & Thompson limit value of $\alpha_{e m}(0)$ & 0.0072993 \\
\hline SWEIN & Value of $\sin ^{2} \theta_{W}$ & 0.2319 \\
\hline $\mathrm{QFCH}(\mathrm{I})$ & Fermion electric charge & \\
\hline $\operatorname{AFCH}(I, J)$ & Fermion weak axial charge & see sect. 14.2 .2 \\
\hline $\operatorname{VFCH}(I, J)$ & Fermion weak vector charge $\mathrm{J}=1: Z, \mathrm{~J}=2: Z^{\prime}$ & \\
\hline ZPRIME & Include a $Z^{\prime}$ in $\gamma^{\star} / Z^{0}$ processes & .FALSE \\
\hline RMASS (202) & Mass of the $Z^{\prime}$ & 500. \\
\hline GAMZP & Width of the $Z^{\prime}$ & 5.0 \\
\hline
\end{tabular}




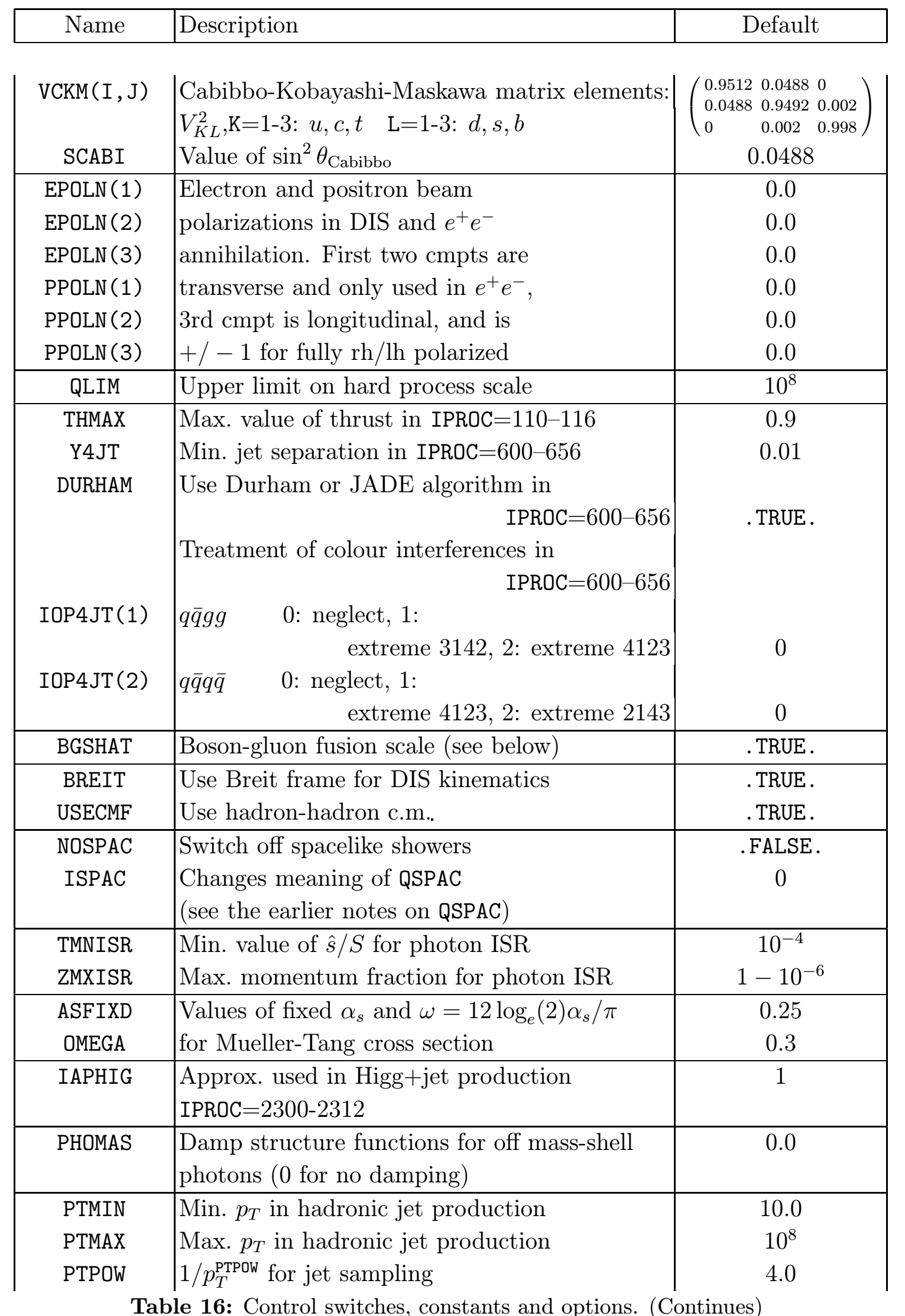




\begin{tabular}{|c|c|c|}
\hline Name & Description & Default \\
\hline Y JMIN & Min. jet rapidity & -8.0 \\
\hline Y JMAX & Max. jet rapidity & +8.0 \\
\hline EMMIN & Min. dilepton mass in Drell-Yan & 10.0 \\
\hline EMMAX & Max. dilepton mass in Drell-Yan & $10^{8}$ \\
\hline EMPOW & $1 / m^{\text {EMPOW }}$ for Drell-Yan sampling & 4.0 \\
\hline Q2MIN & Min. $Q^{2}$ in deep inelastic scattering & 0 \\
\hline Q2MAX & Max. $Q^{2}$ in deep inelastic scattering & $10^{10}$ \\
\hline Q2POW & $1 / Q^{2 \mathrm{Q} 2 \mathrm{POW}}$ for DIS sampling & 2.5 \\
\hline YBMIN & Min. and max. Bjorken- $y$ in DIS & 0.0 \\
\hline YBMAX & & 1.0 \\
\hline WHMIN & $\begin{array}{l}\text { Min. hadronic mass in } \\
\gamma \text {-induced processes (inc. DIS) }\end{array}$ & 0.0 \\
\hline ZJMAX & Max. $z$ in $J / \psi$ production & 0.9 \\
\hline Q2WWMN & Min. and $\max . Q^{2}$ in & 0.0 \\
\hline Q2WWMX & Equivalent Photon Approximation & 4.0 \\
\hline YWWMIN & Min. and max. photon light-cone fraction & 0.0 \\
\hline YWWMAX & in Equiv. Photon Approx. & 1.0 \\
\hline CSPEED & Speed of light in vacuum $(\mathrm{mm} / \mathrm{s})$ & $2.99792 \times 10^{11}$ \\
\hline GEV2NB & Value of $(\hbar c / e)^{2}$ & 389379 \\
\hline $\begin{array}{c}\text { IBSH } \\
\operatorname{IBRN}(1)\end{array}$ & $\begin{array}{l}\text { Number of shots for initial max. weight search } \\
\text { First random number seed for }\end{array}$ & 10000 \\
\hline & $\max$. weight search & 1246579 \\
\hline $\operatorname{IBRN}(2)$ & First random number seed for & \\
\hline & max. weight search & 8447766 \\
\hline NQEV & $\begin{array}{l}\text { Number of entries in Sudakov FF } \\
\text { look-up table }\end{array}$ & 1024 \\
\hline ZBINM & Max. bin size for $z$ in spacelike branching & 0.05 \\
\hline NZBIN & Max. number of $z$ bins in spacelike branching & 100 \\
\hline NBTRY & Max. number of attempts to branch a parton & 200 \\
\hline NCTRY & Max. number of attempts to decay a cluster & 200 \\
\hline NETRY & Max. number of attempts to generate & \\
\hline & required mass & 200 \\
\hline NSTRY & Max. number of attempts at soft subprocess & 200 \\
\hline ACCUR & Precision for soft gaussian integration & $10^{-6}$ \\
\hline RPARTY & R-parity conservation in SUSY & .TRUE. \\
\hline $\begin{array}{l}\text { SUSYIN } \\
\text { LRSUSY }\end{array}$ & $\begin{array}{l}\text { Check to see if SUSY data are already loaded } \\
\text { Unit for reading SUSY data (if needed) }\end{array}$ & $\begin{array}{l}\text {.FALSE. } \\
66\end{array}$ \\
\hline
\end{tabular}

Table 'í $\overline{6}$ : Control switches, constants and options. 


$$
\begin{array}{rl}
\hline \text { IPRINT }=0 & \text { Print program title only } \\
1 & \text { Print selected input parameters } \\
2 & 1+\text { table of particle codes and properties } \\
3 & 2+\text { tables of Sudakov form factors } \\
\hline
\end{array}
$$

Table 17: Printout options.

Printout options are listed in table

The contents of /HEPEVT/ can by printed by calling HWUEPR, those of /HWPART/ (the last parton shower) by calling HWUBPR. The logical variable PRNDEC (default .TRUE. unless NMXHEP > 9999) causes track numbers in event listings to be printed in decimal, or hexadecimal if false. The latter is necessary for very large events such as those generated by the HERBVI package (see above).

The maximum number of errors MAXER refers to errors from which the program cannot recover without killing an event and starting a new one. Such errors are not necessarily a cause for grave concern because the phase space for backward evolution of initial-state showers is complicated and the program may occasionally step outside it (in which case the event weight should be zero anyway). When generating large numbers of events, it is advisable to increase MAXER in proportion, e.g. to MAXEV/100.

See section 18.21 on form factors for details of LRSUD, LWSUD and SUDORD.

The parameter EFFMIN sets the minimum allowed efficiency for the generation of unweighted events. A warning is printed once in every 10/EFFMIN weights if the efficiency is below $10 \times$ EFFMIN, and running is stopped if the efficiency is below EFFMIN.

Variables HARDME and SOFTME invoke hard and soft matrix-element corrections respectively, as described in subsection $3 . \overline{2} \cdot \overline{-}$;

If BGSHAT is . TRUE., the scale used for heavy quark production via boson-gluon fusion in lepton-hadron collisions will be the hard subprocess c.m. energy $\hat{s}$. If it is .FALSE., the scale used will be

$$
\frac{2 \hat{s} \hat{t} \hat{u}}{\hat{s}^{2}+\hat{t}^{2}+\hat{u}^{2}}
$$

except in the case of $J / \psi+g$ production, where $\hat{u}$ is used.

If BREIT is true, the kinematic reconstruction of deep inelastic events takes place in the Breit frame (i.e. the frame where the exchanged boson is purely spacelike, and collinear with the incoming hadron). In fact the reconstruction procedure is invariant under longitudinal boosts, so any frame in which the boson and hadron are collinear would be equivalent, and it is only the transverse part of the boost that has an effect. The BREIT frame option becomes very inaccurate for very small $Q^{2}$. It is therefore only used if $Q^{2}>10^{-4}$ (the lab and Breit frames are anyway equivalent for such small $Q^{2}$ ). If BREIT is false, reconstruction takes place in the lab frame.

If USECMF is true, the entire event record is boosted to the hadron-hadron c.m. frame before event processing, and boosted back afterwards. This means that fixed- 
target simulation can be done in the lab frame, i.e. with PBEAM2 $=0$. For hadronic processes with lepton beams, this boosting is always done, regardless of the value of USECMF.

The quantities from PTMIN to ZJMAX control the region of phase space in which events are generated and importance sampling inside those regions. See section 18.3 .2 on event weights for further details on these quantities and the use of WGTMAX and NOWGT.

If hadronic processes with lepton beams are requested, the photon emission vertex includes the full transverse-momentum-dependent kinematics (the Equivalent Photon Approximation). The variables Q2WWMN and Q2WWMX set the minimum and maximum virtualities generated respectively. For normal simulation, Q2WWMN should be zero, and Q2WWMX should be the largest $Q^{2}$ through which the lepton can be scattered without being detected. The variables YWWMIN and YWWMAX control the range of lightcone momentum fraction generated.

In addition there are options to give different weights to the various flavours of quarks and diquarks, and to resonances of different spins. So far, these options have not been used. See the comments in the initialization routine HWIGIN for details.

\section{Particle data}

From HERWIG version 5.9 onwards, new 8-character particle names have been introduced and the revised 7 digit PDG numbering scheme, as advocated in the LEP2 report [25], has been adopted. All hadron and lepton masses are given to five significant figures whenever possible.

Unstable hadrons from clusters produced in both the hard and soft components of the event decay according to simplified decay schemes, which can be tabulated by specifying the print option IPRINT $=2$. Decays modes are 'invented' where necessary to make the branching ratios add up to $100 \%$. Phase space distributions are assumed except where stated otherwise. See section 3.3 . quark decays. After a $t$, partonic $b$ or quarkonium decay, secondary parton showers are produced by outgoing partons as discussed in ref. [i] 1 in; these are hadronized in the same way as primary jets.

There have been a number of additions/changes to the default hadrons included via HWUDAT. Here the identification of hadrons follows the PDG [3.5;, table 13.2], numbered according to their section 30.

All $\mathrm{S}$ and $\mathrm{P}$ wave mesons are present including the ${ }^{1} \mathrm{P}_{0}$ and ${ }^{3} \mathrm{P}_{1}$ states and many new, excited $\mathrm{B}^{* *}, \mathrm{~B}_{c}$ and quarkonium states. Also all $\mathrm{D}$ wave kaons and some 'light' $\mathrm{I}=3$ states $\left[\pi_{2}, \rho(1700)\right.$ and $\left.\rho_{3}\right]$. All the baryons (singlet/octet/decuplet) containing up to one heavy $(c, b)$ quark are included.

New isoscalars states have been added to try to complete the $1^{3} D_{3}, 1^{1} D_{2}$ and $1^{3} D_{1}$ multiplets (table $1 \overline{1} \overline{8_{1}}$ ). 


\begin{tabular}{|rlr|rlr|}
\hline IDHW & RNAME & IDPDG & IDHW & RNAME & IDPDG \\
\hline 395 & OMEGA_3 & 227 & 396 & PHI_3 & 337 \\
397 & ETA_2(L) & 10225 & 398 & ETA_2(H) & 10335 \\
399 & OMEGA(H) & 30223 & & & \\
\hline
\end{tabular}

Table 18: New isoscalar states.

\begin{tabular}{|rlr|clr|}
\hline IDHW & RNAME & IDPDG & IDHW & RNAME & IDPDG \\
\hline 57 & FH_1 & 20333 & & & \\
293 & FOPO & 9010221 & 294 & FH_00 & 10221 \\
62 & A_O(H)0 & 10111 & 290 & A_00 & 9000111 \\
63 & A_O(H)+ & 10211 & 291 & A_0+ & 9000211 \\
64 & A_O(H)- & -10211 & 292 & A_0- & -9000211 \\
\hline
\end{tabular}

Table 19: Re-identified/replaced states.

Also the states in table $1{ }_{1}^{1} \overline{9}$, have been re-identified/replaced.

The $f_{1}(1420)$ state completely replaces the $f_{1}(1520)$ in the $1^{3} P_{0}$ multiplet, taking over 57 . The $f_{0}(1370)$ (294) replaces the $f_{0}(980)$ (293) in the $1^{3} P_{0}$ multiplet; the latter is retained as it appears in the decays of several other states. The new $a_{0}(1450)$ states (62-64) replace the three old $a_{0}(980)$ states $(290-292)$ in the $1^{3} P_{0}$ multiplet; the latter are kept as they appear in $f_{1}(1285)$ decays.

By default production of the $f_{0}(980)$ and $a_{0}(980)$ states in cluster decays is vetoed.

The mixing angles (in degrees) of all the light, $\mathrm{I}=0$ mesons can now be set using table 20

There were previously some inconsistencies and ambiguities in our conventions for the mixing of flavour 'octet' and 'singlet' mesons. They are now as in table $\underline{2} \overline{1} \overline{1}_{1}^{1}$.

\begin{tabular}{|r|l|}
\hline ETAMIX & $\eta / \eta^{\prime}$ \\
PHIMIX & $\omega / \phi$ \\
H1MIX & $h_{1}(1170) / h_{1}(1380)$ \\
FOMIX & $f_{0}(1300) / f_{0}(980)$ \\
F1MIX & $f_{1}(1285) / f_{1}(1510)$ \\
F2MIX & $f_{2} / f_{2}^{\prime}$ \\
\hline
\end{tabular}

Table 20: Mixing angles.

\begin{tabular}{|cccc|}
\hline Multiplet & Octet & Singlet & Mixing Angle \\
\hline $1^{1} \mathrm{~S}_{0}$ & $\eta$ & $\eta^{\prime}$ & ETAMIX $=-23$. \\
$1^{3} \mathrm{~S}_{1}$ & $\phi$ & $\omega$ & PHIMIX $=+36$. \\
$1^{1} \mathrm{P}_{1}$ & $h_{1}(1380)$ & $h_{1}(1170)$ & H1MIX $=$ ANGLE \\
$1^{3} \mathrm{P}_{0}$ & missing & $f_{0}(1370)$ & FOMIX $=$ ANGLE \\
$1^{3} \mathrm{P}_{1}$ & $f_{1}(1420)$ & $f_{1}(1285)$ & F1MIX $=$ ANGLE \\
$1^{3} \mathrm{P}_{2}$ & $f_{2}^{\prime}$ & $f_{2}$ & F2MIX $=+26$. \\
$1^{1} \mathrm{D}_{2}$ & $\eta_{2}(1645)$ & $\eta_{2}(1870)$ & ET2MIX $=$ ANGLE \\
$1^{3} \mathrm{D}_{1}$ & missing & $\omega(1600)$ & OMHMIX $=$ ANGLE \\
$1^{3} \mathrm{D}_{3}$ & $\phi_{3}$ & $\omega_{3}$ & PH3MIX =+28. \\
\hline
\end{tabular}

Table 21: Mixed meson states. 
After mixing, the quark content of the physical states is given in terms of the mixing angle, $\theta$, by table $22 \overline{2} 2_{1}^{1}$ where $\tan \theta_{0}=\sqrt{2}$.

Hence, using the default value of ANGLE $=\arctan (1 / \sqrt{2})=+35.3$ for $\theta$ gives ideal mixing, that is, the 'octet' state $=s \bar{s}$ and the 'singlet' $=(d \bar{d}+$ $u \bar{u}) / \sqrt{2}$. This choice is important to

\begin{tabular}{|ccc|}
\hline State & $(d \bar{d}+u \bar{u}) / \sqrt{2}$ & $s \bar{s}$ \\
\hline Octet & $\cos \left(\theta+\theta_{0}\right)$ & $-\sin \left(\theta+\theta_{0}\right)$ \\
Singlet & $\sin \left(\theta+\theta_{0}\right)$ & $\cos \left(\theta+\theta_{0}\right)$ \\
\hline
\end{tabular}

Table 22: Quark content of mixed states. avoid large isospin violations in the $1^{3} P_{0}$ and $1^{3} D_{1}$ multiplets in which the octet member is unknown.

Since version 6 contains a large number of supersymmetry processes many new hypothetical particles have been added - see section is.5. These new states do not interfere with the user's ability to add new particles as described below.

The resonance decay tables supplied in the program have also been largely revised. Measured/expected modes with branching fraction at or above 1 per mille are given, including 4 and 5 body decays. To print the new tables call HWUDPR.

The layout of HWUDAT has been altered to make it easier to identify and modify particle properties. Three new arrays have been introduced RLTIM, RSPIN and IFLAV. These are: the particle's lifetime (s), spin, and a code which specifies the flavour content of each hadron - used (in HWURES) to create sets of iso-flavour hadrons for cluster decay. Using the standard numbering of quark flavours the convention is:

- Mesons: $n_{q} n_{\bar{q}}$, e.g. $\pi^{+}: 21, \pi^{-}: 12$;

- Baryons: $\pm n_{q 1} n_{q 2} n_{q 3}$, e.g. $\Xi^{0}: 332, \bar{\Xi}^{0}:-332$ etc. (<0 for antibaryons; digits in decreasing order);

- Light, neutral mesons are identified as: 11 if isovector $\left(\pi^{0}, \rho^{0}, \ldots\right), 33$ if isoscalar $\left(\eta, \eta^{\prime}, \ldots\right)$.

Some parts of the program have been automated so that it is possible for the user to add new particles by specifying their properties via the arrays in /HWPROP/ and /HWUNAM/ and increasing NRES appropriately: this should be done before a call to HWUINC.

As an example, the following lines add an isoscalar, spin-2 state 'STAN' and a (very light) stable toponium state 'BEER' with the decay mode: STAN $\rightarrow$ BEER + BEER + BEER.

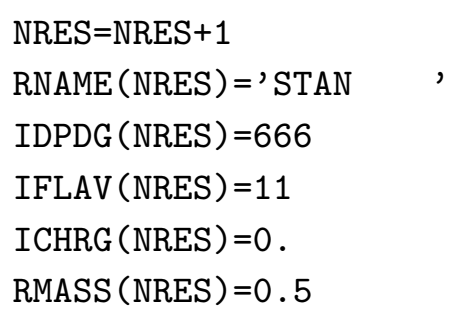




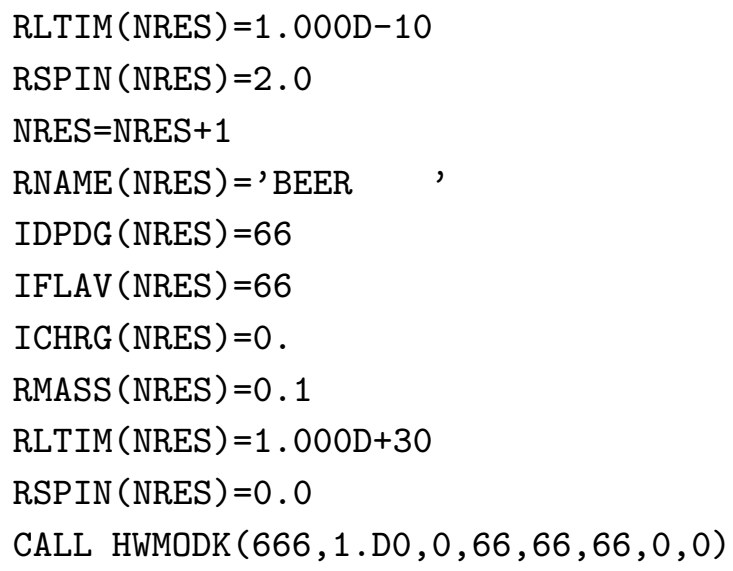

Using the logical arrays VTOCDK and VTORDK the production of specified particles can be stopped in both cluster decays and via the decay of other unstable resonances.

A priori weights for the relative production rates in cluster decays of mesons and baryons differing only via their $\mathrm{S}$ and $\mathrm{L}$ quantum numbers can be supplied using SNGWT and DECWT for singlet (i.e. $\Lambda$-like) and decuplet baryons and REPWT for mesons. The old VECWT now corresponds to $\operatorname{REPWT}(0,1,0)$ and TENWT to $\operatorname{REPWT}(0,2,0)$.

The arrays FBTM, FTOP and FHVY which stored the branching fractions of the bottom, top and heavier quarks' 'partonic' decays are now no longer used. Such decays are specified in the same way as all other decay modes: this permits different decays to be given to individual heavy hadrons. Partonic decays of charm hadrons and quarkonium states are also now supported. As already mentioned, the products' order in a partonic decay mode is significant: see discussion in section 13.31.

The structure of the program has been altered so that the secondary hard subprocess and subsequent fragmentation associated with each partonic heavy hadron decay appears separately. Thus pre-hadronization top quark decays are treated individually, as are any subsequent bottom hadron partonic decays.

Additionally decays of heavy hadrons to exclusive non-partonic final states are supported. No check against double counting from partonic modes is included. However this is not expected to be a major problem for the semi-leptonic and 2-body hadronic modes supplied.

B decays can also be performed by the EURODEC or CLEO Monte Carlo packages. The new variable BDECAY controls which package is used: 'HERW' for HERWIG; 'EURO' for EURODEC; 'CLEO' for CLEO. The EURODEC package can be obtained from the CERN library. The CLEO package is available by kind permission of the CLEO collaboration.

An array NME has been introduced to enable a possible matrix element to be specified for each decay mode.

- $\operatorname{NME}=0$ : Isotropic decay

- $\operatorname{NME}=100:$ Free particle $(V-A) *(V-A),\left(p_{0} \cdot p_{2}\right)\left(p_{1} \cdot p_{3}\right)$ 
- $\operatorname{NME}=101:$ Bound quark $(V-A) *(V-A),\left(p_{0} \cdot p_{2}\right)\left(p_{1} \cdot\left[p_{3}-x_{s} p_{0}\right]\right), x_{s}=$ $m_{Q} / M_{0}=$ spectator quark momentum fraction.

- NME $=130$ : Ore and Powell ortho-positronium matrix element for: onium $\rightarrow$ $g g+g / \gamma$.

- NME $=200$ : Reserved for free-particle $t \rightarrow b$ quark decay through a scalarfermion-fermion current.

- $\operatorname{NME}=300:$ Gaugino and gluino three-body $R_{\mathrm{p}}$ decays. This also implements the angular ordering procedure in the $R_{\mathrm{p}}$ gluino decays.

The list of matrix elements currently supported is modest; users are urged to contact an author to have others implemented.

A $Z^{\prime}$ has been introduced with PDG code 32, HERWIG identifier 202, default mass $500 \mathrm{GeV}$, width GAMZP (default $5 \mathrm{GeV}$ ) and name 'ZOPR '. It is invoked by setting ZPRIME $=$. TRUE. (default .FALSE.).

The decay tables can be written to/read from a file by using HWIODK, adopting the format advocated in the LEP2 report [2필. of particles the HERWIG numbers or character names can be used. This permits easy alteration of the decay tables. In HWUINC a call is made to HWUDKS which sets up HERWIG internal pointers and performs some basic checks of the decay tables. Each decay mode must conserve charge and be kinematically allowed and not contain vetoed decay products. The sum of all branching ratios is set to 1 for all particles. Also a warning is printed if an antiparticle does not have all the charge conjugate decays modes of the particle.

HWMODK enables changes to the decay tables to be made by alterating or adding single decay modes including on an event-by-event basis. This can be done before calling HWUINC, in which case when altering the branching ratio and/or matrix element code of an existing mode a warning is given of a duplicate second mode which superseeds the first. Branching ratios set below $10^{-6}$ are eliminated, whilst if one mode is within $10^{-6}$ of unity all other modes are removed. Note that some forethought is required if the branching ratios of two modes of the same particle are changed since the operation of rescaling the branching ratio sum to unity causes a non-commutativity in the order of the calls.

It is possible to create particle property and event listings in any combination of 3 formats - standard ASCII, LTEX or html. These options are controlled by the logical variables PRNDEF (default .TRUE.) PRNTEX (default .FALSE.) and PRNWEB (default .FALSE.). The ASCII output is directed to stout (screen/log file) as in previous versions. When a listing of particle properties is requested (IPRINT.GE.2 or HWUDPR is called explicitly) then the following files are produced:

If (PRNTEX): HW_decays.tex 


\section{If (PRNWEB): HW_decays/index.html \\ /PART0000001.html etc.}

The HW_decays.tex file is written to the working directory whilst the many $* *$.html files appear in the sub-directory HW_decays/ which must have been created previously. Paper sizes and offsets for the $\mathrm{AT}_{\mathrm{EX}} \mathrm{Xutput}$ are stored at the top of the block data file HWUDAT: they may need modifying to suit a particular printer. When event listings are requested (NEVHEP.LE.MAXPR or HWUEPR is called explicitly) the following files are created in the current working directory:

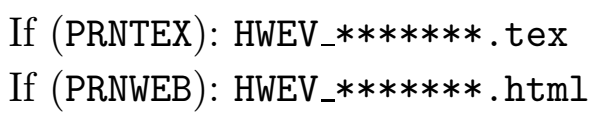

where $* * * * * * *=0000001$ etc. is the event number.

Note that the html file automatically makes links to the index.html file of particle properties, assumed to be in the HW_decays sub-directory.

A new integer variable NPRFMT (default 1) has been introduced to control how many significant figures are shown in each of the 3 event outputs. Basically NPRFMT=1 gives short compact outputs whilst NPRFMT=2 gives long formats.

Note that all the $\mathrm{AT}_{\mathrm{E}} \mathrm{X}$ files use the package longtable. sty to format the tables. Also if NPRFMT=2 or PRVTX=. TRUE. then the $\mathrm{IATEX}_{\mathrm{E}}$ files are designed to be printed in landscape mode.

\section{Structure and output}

\subsection{Main program}

The main program HWIGPR has the following form:

PROGRAM HWIGPR

C---COMMON BLOCKS ARE INCLUDED AS FILE HERWIG62.INC

INCLUDE 'HERWIG62. INC'

INTEGER N

EXTERNAL HWUDAT

C---MAX NUMBER OF EVENTS THIS RUN

MAXEV $=100$

C---BEAM PARTICLES

PART1 $=$ ' $P$ '

PART2 = ' $\mathrm{P}$ '

C---BEAM MOMENTA

PBEAM1 $=7000$.

PBEAM2 $=$ PBEAM1

C---PROCESS 
IPROC $=3000$

C---INITIALISE OTHER COMMON BLOCKS

CALL HWIGIN

C---USER CAN RESET PARAMETERS AT THIS POINT,

C OTHERWISE DEFAULT VALUES IN HWIGIN WILL BE USED. PRVTX $=$. FALSE .

$M A X P R=0$

MAXER $=$ MAXEV $/ 100$

C N.B. TO READ SUDAKOV FORM FACTOR FILE ON UNIT 77

C INSERT THE FOLLOWING TWO LINES IN SUBSEQUENT RUNS

C LRSUD $=77$

C LWSUD $=0$

C---RESET RANDOM NUMBER SEEDS IF YOU LIKE

$\operatorname{NRN}(1)=870875355$

$\operatorname{NRN}(2)=138323456$

C---INPUT SUSY PARTICLE (AND TOP QUARK) DATA

CALL HWISSP

C---COMPUTE PARAMETER-DEPENDENT CONSTANTS

CALL HWUINC

C---CALL HWUSTA TO MAKE ANY PARTICLE STABLE

CALL HWUSTA ('PIO ')

C---USER'S INITIAL CALCULATIONS

CALL HWABEG

C---INITIALISE ELEMENTARY PROCESS

CALL HWEINI

C---LOOP OVER EVENTS

DO $100 \mathrm{~N}=1$, MAXEV

C---INITIALISE EVENT

CALL HWUINE

C---GENERATE HARD SUBPROCESS

CALL HWEPRO

C---GENERATE PARTON CASCADES

CALL HWBGEN

C---DO HEAVY OBJECT DECAYS

CALL HWDHOB

C---DO CLUSTER FORMATION

CALL HWCFOR

C---DO CLUSTER DECAYS

CALL HWCDEC

C---DO UNSTABLE PARTICLE DECAYS

CALL HWDHAD

C---DO HEAVY FLAVOUR HADRON DECAYS

CALL HWDHVY 


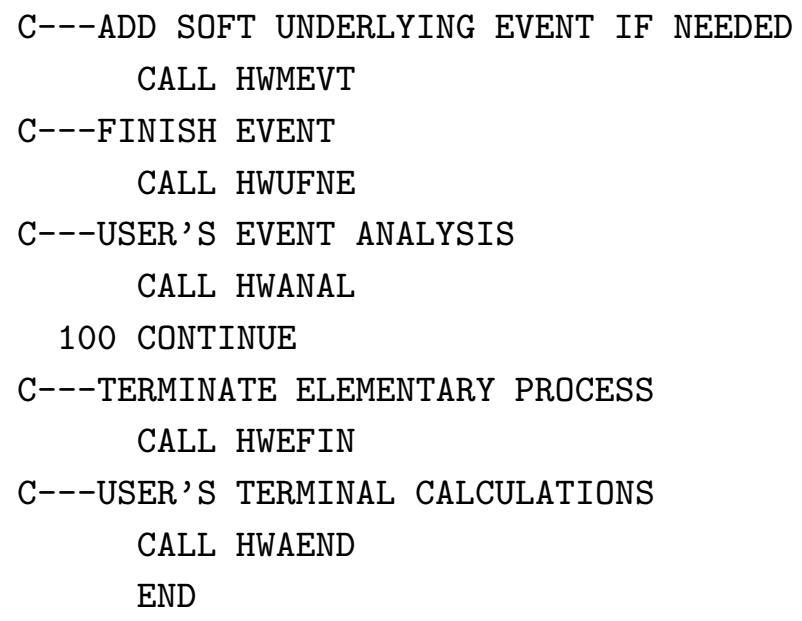

The declaration EXTERNAL HWUDAT is recommended to help the linker with finding the block data on some systems.

Various phases of the simulation can be suppressed by deleting the corresponding subroutine calls, or different subroutines may be substituted. For example, in nonSUSY studies the call to HWISSP should be omitted, and in studies at the parton level everything from CALL HWCFOR to CALL HWMEVT can be omitted.

Note that the functionality of the routine HWUINE in earlier versions has now been split between it and a new routine, HWUFNE. A call to the latter must be made between the calls to HWMEVT and HWANAL. A check is built in to prevent execution if this is not done.

The analysis routine HWANAL should always begin with the line

IF (IERROR.NE.0) RETURN

since if an event is cancelled, each of the routines is still called in turn until reaching the end of the main loop.

\subsection{Form factor file}

HERWIG uses look-up tables of Sudakov form factors for the evolution of initial- and final-state parton showers. These can be read from an input file rather than being recomputed each time. The reading, writing and computing of form factor tables is controlled by integer parameters LRSUD and LWSUD (table $2 \overline{3} \overline{1}$ ).

\begin{tabular}{|ll|}
\hline LRSUD $=\mathrm{N}>0$ & Read form factors for this run from unit $\mathrm{N}$ \\
LRSUD $=0$ & Compute new form factor tables for this run \\
LRSUD $<0$ & Form factor tables are already loaded \\
LWSUD $=\mathrm{N}>0$ & Write form factors on unit $\mathrm{N}$ for future use \\
LWSUD $=0$ & Do not write new form factor tables \\
\hline
\end{tabular}

Table 23: Form factor read/write options. 
The option LRSUD $<0$ allows the program to be initialized several times in the same run (e.g. to generate various event types) without recomputing or rereading form factors.

Note that the Sudakov form factors depend on the parameters QCDLAM, VQCUT, VGCUT, NCOLO, NFLAV, RMASS(13) and RMASS $(i)$ for $i=1, \ldots$, NFLAV. Consequently form factor tables must be recomputed every time any of these parameters is changed. These parameters are written/read with the form factor tables and checks are performed to ensure consistency.

The parton showering algorithm uses the two-loop running coupling, with matching at each flavour threshold. However, the Sudakov table can be computed with either the one-loop or two-loop form, according to the variable SUDORD (=1 or 2 respectively, default=1). If SUDORD $=1$ the two-loop value is recovered using the veto algorithm in the shower, whereas if SUDORD $=2$ no vetoes are used in the final-state evolution. This means that the relative weight of any shower configuration can be calculated in a closed form, hence that showers can be 'forced'.

To next-to-leading order the two possibilities should be identical, but they differ at beyond-NLO, so some results may change a little. The most noticeable difference is that the form factor table takes a factor of about five times longer to compute with SUDORD $=2$ than with 1 .

When $\operatorname{SUDORD}=2$, no veto is needed for gluon splitting to quarks. This means that no vetoes are needed for final state showering, except for the previously-mentioned transverse momentum cut. The removal of vetoes allows preselection of the flavours that a jet will contain, giving a huge increase in the efficiency of rare process simulation $\left[\begin{array}{l}\overline{7} \overline{0} \\ \underline{1}\end{array}\right]$.

\subsection{Event data}

/HEPEVT/ is the LEP standard common block containing current event data (table 2

\begin{tabular}{|ll|}
\hline NEVHEP & event number \\
$\operatorname{NHEP}$ & number of entries for this event \\
$\operatorname{ISTHEP}(\mathrm{I})$ & status of entry I (see below) \\
$\operatorname{IDHEP}(\mathrm{I})$ & identity of entry I (Particle Data Group code) \\
$\operatorname{JMOHEP}(1, \mathrm{I})$ & pointer to first mother of entry I (see below) \\
$\operatorname{JMOHEP}(2, \mathrm{I})$ & pointer to second mother of entry I (see below) \\
$\operatorname{JDAHEP}(1, \mathrm{I})$ & pointer to first daughter of entry I (see below) \\
$\operatorname{JDAHEP}(2, \mathrm{I})$ & pointer to last daughter of entry I (see below) \\
$\operatorname{PHEP}(*, \mathrm{I})$ & $\left(p_{x}, p_{y}, p_{z}, E, M\right)$ of entry I: $M=\operatorname{sign}\left(\sqrt{\operatorname{abs}\left(m^{2}\right)}, m^{2}\right)$ \\
$\operatorname{VHEP}(*, \mathrm{I})$ & $(x, y, z, t)$ of production vertex of entry I (see sect. $\left.{ }^{-3.7_{1}^{\prime}}\right)$ \\
\hline
\end{tabular}

Table 24: Current event data. 
All momenta are given in $\mathrm{GeV} / c$ in the laboratory frame, in which the input beam momenta are PBEAM1 and PBEAM2 as specified by the user and point along the $+z$ and $-z$ directions respectively. Final state particles have $\operatorname{ISTHEP}(\mathrm{I})=1$. See section $8 . \overline{3} .1$ for a complete list of the special status codes used by HERWIG.

The identity codes IDHEP are almost as recommended by the LEP Working Group [25], i.e. the revised PDG [35] numbers where defined, IDHEP $=91$ for clusters, 94 for jets, and 0 for objects with no PDG code. The only exception is our use of IDHEP $=26$ for the lightest MSSM Higgs boson, to distinguish it from the SM Higgs boson (PDG code 25). In addition, the 'generator-specific' (IDHEP=81100) codes 98 and 99 are used for remnant photons and nucleons, respectively (see section $3 . \overline{6} . \overline{2}$.

HERWIG also has its own internal identity codes IDHW(I), stored in /HWEVNT/. The utility subroutine HWUIDT translates between HERWIG and PDG identity codes. See section $1 \overline{1} \cdot \overline{1}$ ' for further details.

The mother and daughter pointers are standard, except that $\operatorname{JMOHEP}(2, \mathrm{I})$ and $\operatorname{JDAHEP}(2, \mathrm{I})$ for a parton are its colour mother and colour daughter, i.e. the partons to which its colour and anticolour are connected, respectively. For this purpose the primary partons from a hard subprocess are all regarded as outgoing (see examples

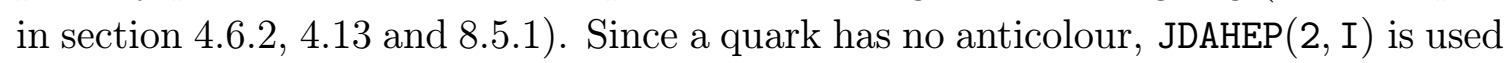
to point to its flavour partner. Similarly for $\operatorname{JMOHEP}(2, \mathrm{I})$ in the case of an antiquark.

In addition to entries representing partons, particles, clusters etc, /HEPEVT/ contains purely informational entries representing the total centre-of-mass momentum, hard and soft subprocess momenta, etc. See section 18.3 , for the corresponding status codes.

Information from all stages of event processing is retained in /HEPEVT/ so the same particle may appear several times with different status codes. For example, an outgoing parton from a hard scattering (entered initially with status 113 or 114) will appear after processing as an on-mass-shell parton before QCD branching (status 123,124), an off-mass-shell entry representing the flavour and momentum of the outgoing jet (status 143,144), and a jet constituent (157). It might also appear again in other contexts, e.g. as a spectator in a heavy flavour decay (status 154,160).

Incoming partons (entered with status 111 or 112, changed to 121 or 122 after branching) give rise to spacelike jets (status 141 or 142) with $m^{2}<0$, indicated by $\operatorname{PHEP}(5$, IHEP $)<0$, due to the loss of momentum via initial-state bremsstrahlung. The same applies in principle to incoming leptons, but in that case emission of at most a single photon is permitted from each initial-state lepton and the off-shell lepton is given status code 3 (see section $18.3 . \overline{1}$ ).

Each parton jet begins with a status 141-144 jet entry giving the total flavour and momentum of the jet. The first mother pointer of this entry gives the location of the parent hard parton, while the second gives that of the subprocess centre-ofmass momentum. If QCD branching has occurred, this is followed by a lightlike 
CONE entry, which fixes the angular extent of the jet and its azimuthal orientation relative to the parton with which it interferes. The interfering parton is listed as the second mother of the cone. Next come the actual constituents of the jet. If no branching has occurred, there is no cone and the single jet constituent is the same as the jet.

Since version 5.1, the event record has been modified to retain entries for all partons before hadronization (with status ISTHEP=2). During hadronization, the gluons are split into quark-antiquark, while other partons are copied to a location (indicated by $\operatorname{JDAHEP}\left(1,{ }^{*}\right)$ ) where their momenta may be shifted slightly, to conserve momentum, during heavy cluster splitting. Previously the original momenta were shifted, so momentum appeared not to be conserved at the parton level.

\subsubsection{Status codes}

A complete list of currently-used HERWIG status codes is given below (table $22 \overline{5}-1)$. Many are used only in intermediate stages of event processing. The most important for users are probably 1 (final-state particle), 101-3 (initial state), 141-4 (jets), and 199 (decayed $b$-flavoured hadrons).

For technical reasons, some HERWIG status codes ISTHEP between 153 and 165 have changed their meanings since version 5.1.

The event status ISTAT in common /HWEVNT/ is roughly ISTHEP - 100 where ISTHEP is the status of entries being processed. For completed events, ISTAT $=100$.

\begin{tabular}{|c|l|}
\hline ISTHEP & Description \\
\hline 1 & final state particle \\
2 & parton before hadronization \\
3 & documentation line \\
\hline 100 & cone limiting jet evolution \\
101 & 'beam' (beam 1) \\
102 & 'target' (beam 2) \\
103 & overall centre of mass \\
\hline 110 & unprocessed hard process c.m. \\
111 & unprocessed beam parton \\
112 & unprocessed target parton \\
113 & unproc. first outgoing parton \\
114 & unproc. other outgoing parton \\
115 & unprocessed spectator parton \\
\hline $120-25$ & as 110-15, after processing \\
\hline 130 & lepton in jet (unboosted) \\
$131-34$ & as $141-44$, unboosted to c.m. \\
135 & spacelike parton (beam, unboosted) \\
\hline
\end{tabular}

Table $\mathbf{2} \overline{5}$;: Status codes. (Continues) 


\begin{tabular}{|l|l|}
\hline ISTHEP & Description \\
\hline
\end{tabular}

\begin{tabular}{|c|l|}
136 & spacelike parton (target,unboosted) \\
137 & spectator (beam, unboosted) \\
138 & spectator (target, unboosted) \\
139 & parton from branching (unboosted) \\
140 & parton from gluon splitting (unboosted) \\
\hline $141-44$ & jet from parton type 111-14 \\
$145-50$ & as 135-40 boosted, unclustered \\
\hline 151 & as 159, not yet clustered \\
152 & as 160, not yet clustered \\
153 & spectator from beam \\
154 & spectator from target \\
155 & heavy quark before decay \\
156 & spectator before heavy decay \\
157 & parton from QCD branching \\
158 & parton from gluon splitting \\
159 & parton from cluster splitting \\
160 & spectator after heavy decay \\
\hline 161 & beam spectator after gluon splitting \\
162 & target spectator after gluon splitting \\
163 & other cluster before soft process \\
164 & beam cluster before soft process \\
165 & target cluster before soft process \\
167 & unhadronized beam cluster \\
168 & unhadronized target cluster \\
\hline 170 & soft process centre of mass \\
171 & soft cluster (beam, unhadronized) \\
172 & soft cluster (target, unhadronized) \\
173 & soft cluster (other, unhadronized) \\
\hline 181 & beam cluster (no soft process) \\
182 & target cluster (no soft process) \\
183 & hard process cluster (hadronized) \\
184 & soft cluster (beam, hadronized) \\
185 & soft cluster (target, hadronized) \\
186 & soft cluster (other, hadronized) \\
\hline $190-93$ & as 195-98, before decays \\
195 & direct unstable non-hadron \\
196 & direct unstable hadron (1-body clus.) \\
197 & direct unstable hadron (2-body clus.)
\end{tabular}

Table 25:5: Status codes. (Continues) 


\begin{tabular}{|l|l|}
\hline ISTHEP & Description \\
\hline 198 & indirect unstable hadron or lepton \\
199 & decayed heavy flavour hadron \\
\hline 200 & neutral B meson, flavour at prod'n \\
\hline
\end{tabular}

Table '들: Status codes.

\subsubsection{Event weights}

The default is to generate unweighted events (EVWGT $=$ AVWGT). Then event distributions are generated by computing a weight proportional to the cross section and comparing it with a random number times the maximum weight. Set WGTMAX to the maximum weight, or to zero for the program to compute it. If a weight greater than WGTMAX is generated during execution, a warning is printed and WGTMAX is reset. If this occur too often, output event distributions could be distorted.

To generate weighted events, set NOWGT $=$.FALSE. in common /HWEVNT/.

In QCD hard scattering and heavy flavour and direct photon production (IPROC $=$ 1500-1800) the transverse energy distribution of weighted events (or the efficiency for unweighted events) can be varied using the parameters PTMIN, PTMAX and PTPOW.

Similarly in Drell-Yan processes (IPROC $=1350$ etc.) the lepton pair mass distribution is controlled by the parameters EMMIN, EMMAX and EMPOW, and in deep inelastic scattering the $Q^{2}$ distribution depends on Q2MIN, Q2MAX and Q2POW.

Data on weights generated are output at the end of the run. The mean weight is an estimate of the cross section (in nanobarns) integrated over the region used for event generation. Note that the mean weight is the sum of weights divided by the total number of weights generated, not the total number of events.

The maximum weight is now always printed in full precision. This is needed to be sure of generating the same events in repeated runs.

\subsection{Error conditions}

Certain combinations of input parameters may lead to problems in execution. HERWIG tries to detect these and print a warning. Errors during execution are dealt with by HWWARN, which prints the calling subprogram and a code and takes appropriate action. In general, the larger the code the more serious the problem. Refer to the source code to find out why HWWARN was called. It is important to note the subprogram from which the call was issued: many different subprograms use the same error code, but each code is unique within a given subprogram.

Events can be rerun by setting the random number seeds NRN (1) and NRN(2) to the values given in the error message or event dump, and MAXWGT to the maximum weight encountered in the run. The contents of /HEPEVT/ can by printed by calling HWUEPR, those of /HWPART/ (the last parton shower) by calling HWUBPR. 
Note that if WGTMAX is increased during event generation, so that this type of message is printed:

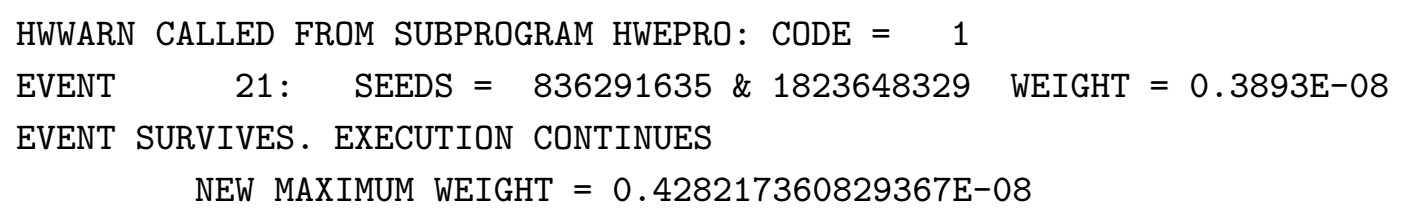

then to regenerate any later events, WGTMAX must be set to the printed value, as well as setting NRN to the appropriate seeds.

Examples of error messages are:

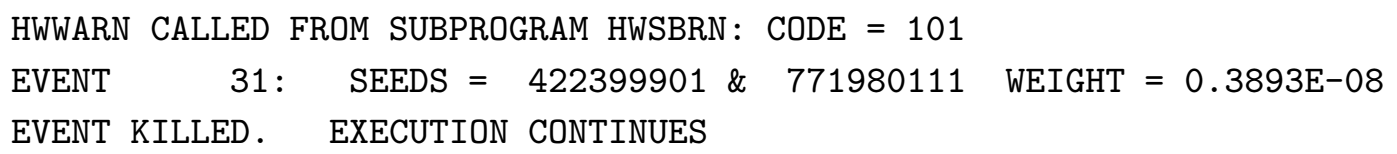

Spacelike (initial-state) parton branching had no phase space. This can happen due to cutoffs which are slightly different in the hard subprocess and the parton shower. Action taken: program throws away this event and starts a new one.

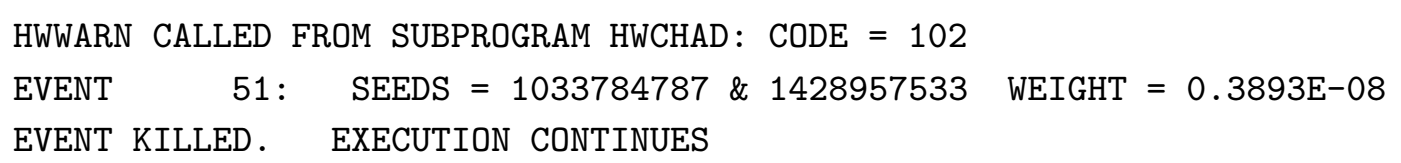

A cluster has been formed with too low a mass to represent any hadron of the correct flavour, and there is no colour-connected cluster from which the necessary additional mass could be transferred.

Action taken: program throws away this event and starts a new one.

HWWARN CALLED FROM SUBPROGRAM HWUINE: CODE $=200$

EVENT SURVIVES. RUN ENDS GRACEFULLY

CPU time limit liable to be reached before generating MAXEV events.

Action taken: skips to terminal calculations using existing events.

HWWARN CALLED FROM SUBPROGRAM HWBSUD: CODE $=500$

RUN CANNOT CONTINUE

The table of Sudakov form factors read on unit LRSUD does not extend to the maximum momentum scale QLIM specified for this run.

Action taken: run aborted. The user must either reduce QLIM or set LRSUD to zero to make a bigger table (set LWSUD non-zero to write it).

HWWARN CALLED FROM SUBPROGRAM HWBSUD: CODE $=515$

RUN CANNOT CONTINUE

The table of Sudakov form factors read on unit LRSUD is for a different value of a relevant parameter (in this case the $b$ quark mass).

Action taken: run aborted. The user must make a new table (set LWSUD non-zero to write it). 


\subsection{Sample output}

This is the output from the main program listed in section '8.1', with no event printout or user analysis.

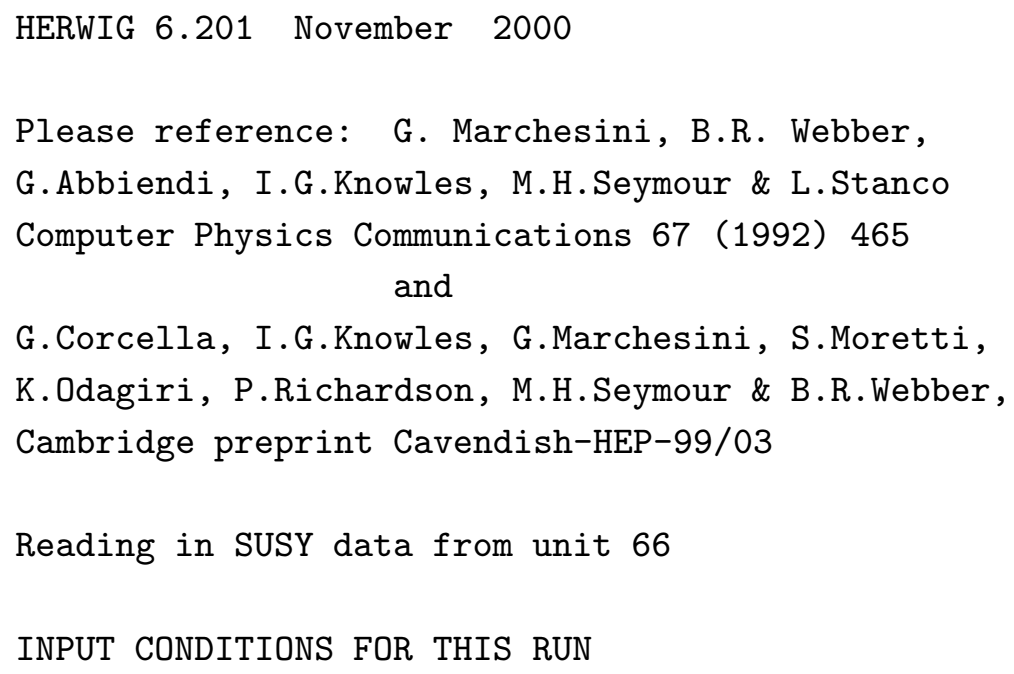


B_s: Delt-M/Gam $=10.00$ Delt-Gam $/ 2 *$ Gam $=0.2000$

PDFLIB NOT USED FOR BEAM 1

PDFLIB NOT USED FOR BEAM 2

Checking consistency of particle properties

Checking consistency of decay tables

Line $\quad 1$ is the same as line 2632

Take BR 0.333 and ME code 100 from second entry

Line 2 is the same as line 2633

Take BR 0.333 and ME code 100 from second entry

Line 3 is the same as line 2634

Take BR 0.111 and ME code 100 from second entry

Line 4 is the same as line 2635

Take BR 0.111 and ME code 100 from second entry

Line $\quad 5$ is the same as line 2636

Take BR 0.111 and ME code 100 from second entry

Line $\quad 6$ is the same as line 2637

Take BR 0.333 and ME code 100 from second entry

Line $\quad 7$ is the same as line 2638

Take BR 0.333 and ME code 100 from second entry

Line $\quad 8$ is the same as line 2639

Take BR 0.111 and ME code 100 from second entry

Line 9 is the same as line 2640

Take BR 0.111 and ME code 100 from second entry

Line 10 is the same as line 2641

Take BR 0.111 and ME code 100 from second entry

WRITING SUDAKOV TABLE ON UNIT 77

PARTICLE TYPE $21=$ PIO SET STABLE

INITIAL SEARCH FOR MAX WEIGHT

$\begin{array}{rrr}\text { PROCESS CODE IPROC } & 3000 \\ \text { RANDOM NO. SEED 1 } & 1246579 \\ \text { SEED 2 } & 8447766 \\ \text { NUMBER OF SHOTS }= & 10000 \\ \text { NEW MAXIMUM WEIGHT }= & 6.8860089763663943 \mathrm{E}-04 \\ \text { NEW MAXIMUM WEIGHT }= & 4.4321257831816674 \mathrm{E}-03 \\ \text { NEW MAXIMUM WEIGHT }= & 1.2204736919093729 \mathrm{E}-02\end{array}$




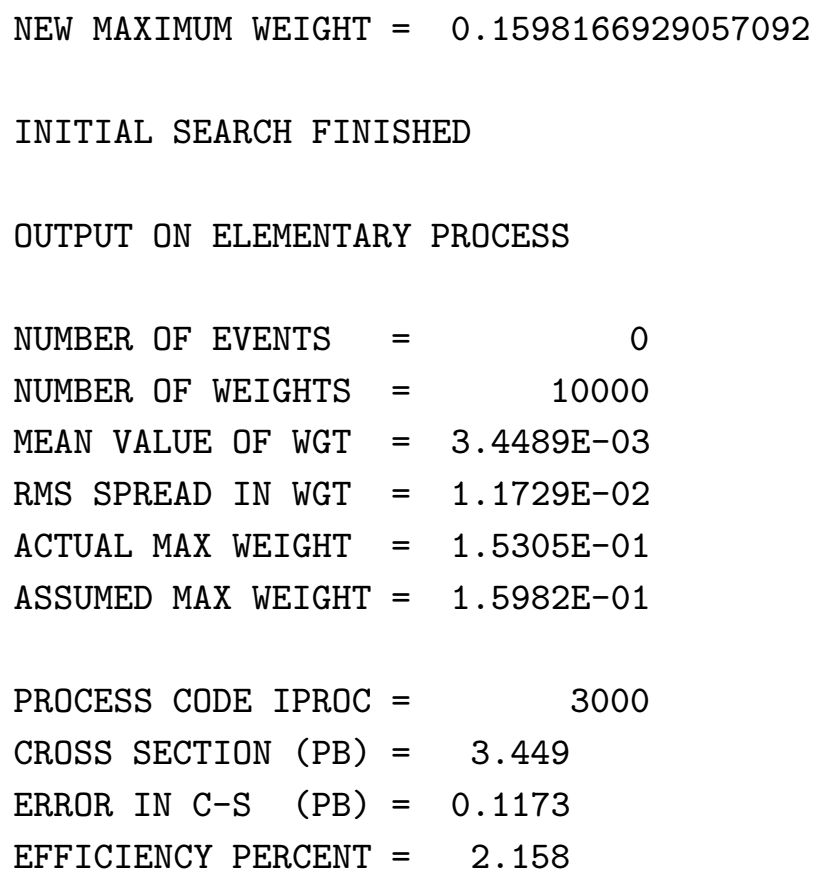

HWWARN CALLED FROM SUBPROGRAM HWDHOB: CODE $=1$

EVENT $40: \quad$ SEEDS $=1586534729 \& 1236586612$ WEIGHT $=0.3449 \mathrm{E}-02$

EVENT SURVIVES. EXECUTION CONTINUES

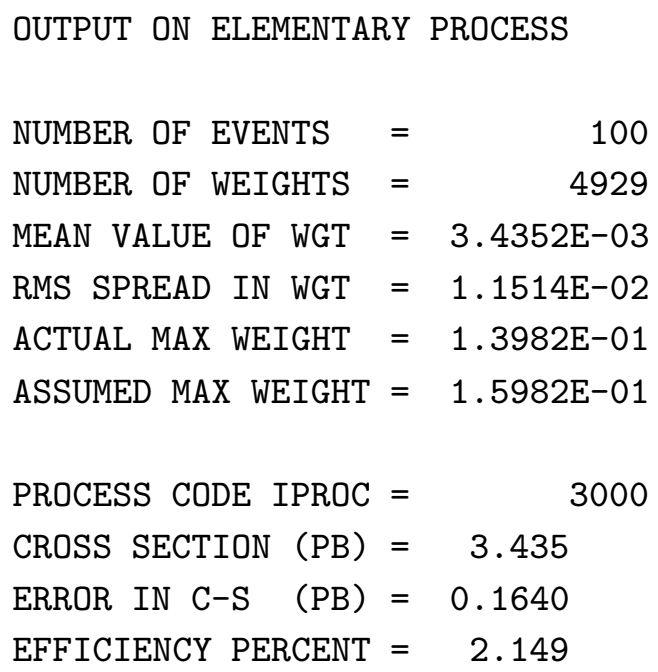

\subsubsection{Guide to sample output}

See ref. [i] for a full discussion of the basic features of HERWIG output, including a listing of a sample event. Here we point out only some new features in comparison to version 5.1. 
The beam particles, their energies and the process code IPROC $=3000$ indicate the SUSY process of squark and/or gluino production at LHC. The call to HWISSP triggers a request for a SUSY particle data file, in the format specified in section which is read in from the default unit. In this case the file corresponds to the second LHC SUGRA point discussed in section

First the program lists the main relevant input parameters, including $B_{d}$ and $B_{s}$ mixing parameters. Parton distributions were not requested from the PDFLIB library

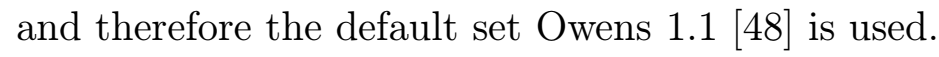

Next the program performs some basic checks on the particle data provided. Here it finds that the input file read by HWISSP contains top quark decay modes which duplicate the default modes stored in HWUDAT. The branching ratios and matrix element codes are accordingly updated to those in the input file.

After an initial search for the maximum weight, the program prints its estimate of the relevant cross section and the expected Monte Carlo efficiency for event generation.

During event generation, a non-fatal (code 1) warning message is issued by subroutine HWDHOB, which handles the decay of heavy objects, including SUSY particles. Consulting the source code, one finds that this indicates that a selected decay mode could not be generated in NETRY (default $=200$ ) attempts at sampling the BreitWigner mass distributions of the decay products. This is a common warning in SUSY processes, since a decay mode may be very close to, or even below, the nominal threshold. In this case the program goes back and chooses another decay mode, according to the tabulated branching ratios.

In the course of event generation a new cross section estimate is generated, which is printed together with the actual Monte Carlo efficiency at the end of the run.

\subsection{Subroutine descriptions}

We give in table $12 \overline{2} \overline{6}$, a list of all subroutines with their functions. Note that the third letter of the name usually follows a rough classification scheme.

\begin{tabular}{|c|l|}
\hline Name & Description \\
\hline & Main program and initialization \\
\hline HWIGPR & Main program \\
HWIGIN & Default initializations \\
\hline & Reading/writing/altering decay modes \\
\hline HWIODK & Inputs/outputs formatted decay tables \\
HWISSP & Inputs supersymmetric particle data \\
HWMODK & Modifies or adds an individual decay mode \\
\hline & User-provided analysis routines \\
\hline HWABEG & Initializes user's analysis
\end{tabular}

Table 26: Subroutines. (Continues) 


\begin{tabular}{|c|c|}
\hline Name & Description \\
\hline HWAEND & Terminates user's analysis \\
\hline \multirow[t]{2}{*}{ HWANAL } & Performs user's analysis on event \\
\hline & Parton branching with interfering gluons \\
\hline HWBAZF & Computes azimuthal correlation functions \\
\hline HWBCON & Makes colour connections between jets \\
\hline HWBDED & Correction to the 'dead zone' in $e^{+} e^{-}$ \\
\hline HWBDIS & Correction to the 'dead zone' in DIS \\
\hline HWBDYP & Correction to the 'dead zone' in Drell-Yan \\
\hline HWBFIN & Transfers external lines of jet to /HEPEVT/ \\
\hline HWBGEN & Finds unevolved partons and generates jets \\
\hline HWBJCO & Combines jets with correct kinematics \\
\hline HWBMAS & Computes masses and trans. momenta in jet \\
\hline HWBRAN & Generates a timelike parton branching \\
\hline HWBRCN & Replaces HWBCON if R-parity is violated \\
\hline HWBRC1 & Finds colour partner in gluino decay \\
\hline HWBRC2 & Finds colour partner in jet \\
\hline HWBSPA & Computes momenta in spacelike jet \\
\hline HWBSPN & Computes spin density/decay matrices \\
\hline HWBSU1 & First term in quark Sudakov form factor \\
\hline HWBSU2 & Second term in quark Sudakov form factor \\
\hline HWBSUD & Computes (or reads) Sudakov form factors \\
\hline HWBSUG & Integrand in gluon Sudakov form factor \\
\hline HWBSUL & Logarithmic part of Sudakov form factor \\
\hline HWBTIM & Computes momenta in timelike jet \\
\hline HWBTOP & Correction to the 'dead zone' in top decay \\
\hline \multirow[t]{2}{*}{ HWBVMC } & Virtual mass cutoff for parton type ID \\
\hline & Cluster hadronization model \\
\hline HWCBCT & Cuts a massive baryon cluster in two \\
\hline HWCBVI & Clusters quarks from a $\not B$ interaction \\
\hline HWCBVT & Finds which $\not B$ interaction partons came from \\
\hline HWCCCC & Finds colour connections after gluon splitting if $\beta$ \\
\hline HWCCUT & Cuts a massive cluster in two \\
\hline HWCDEC & Decays clusters into primary hadrons \\
\hline HWCFLA & Sets up flavours for HWCHAD \\
\hline HWCFOR & Forms clusters \\
\hline HWCGSP & Splits gluons \\
\hline HWCHAD & Decays a cluster into one or two hadrons \\
\hline
\end{tabular}

Table 20: Subroutines. (Continues) 


\begin{tabular}{|c|c|}
\hline Name & Description \\
\hline & Particle and heavy quark decays \\
\hline HWDBOS & Finds and decays $\mathrm{W}$ and $\mathrm{Z}$ bosons \\
\hline HWDBOZ & Chooses decay mode of $\mathrm{W}$ and $\mathrm{Z}$ bosons \\
\hline HWDCHK & Checks given decay mode is self-consistent \\
\hline HWDCLE & Interface to CLEO package for B decays \\
\hline HWDEUR & Interface to EURODEC package for B decays \\
\hline HWDFIV & Generates a five-body decay \\
\hline HWDFOR & Generates a four-body decay \\
\hline HWDHAD & Generates decays of unstable hadrons \\
\hline HWDHGC & Higgs $\rightarrow \gamma \gamma$ decay \\
\hline HWDHGF & Higgs $\rightarrow W^{+} W^{-}$decay \\
\hline HWDHIG & Finds and decays Higgs bosons \\
\hline HWDHOB & Finds and decays heavy objects \\
\hline HWDHVY & Finds and decays heavy flavours \\
\hline HWDIDP & Chooses a parton for HWDHVY \\
\hline HWDPWT & Phase space three-body decay weight \\
\hline HWDRCL & Colour connections for a B SUSY decay \\
\hline HWDRME & Main $R_{\mathrm{p}}$ 3-body decay matrix element subroutine \\
\hline HWDRM1 & $R_{\mathrm{p}}$ 3-body decay matrix element subroutine \\
\hline HWDRM2 & $R_{\mathrm{p}}$ 3-body decay matrix element subroutine \\
\hline HWDRM3 & $R_{\mathrm{p}}$ 3-body decay matrix element subroutine \\
\hline HWDRM4 & $R_{\mathrm{p}}$ 3-body decay matrix element subroutine \\
\hline HWDRM5 & $R_{\mathrm{p}}$ 3-body decay matrix element subroutine \\
\hline HWDTHR & Generates a three-body decay \\
\hline HWDTOP & Decides whether to decay top quark \\
\hline HWDTWO & Generates a two-body decay \\
\hline HWDWWT & Weak $(V-A)$ three-body decay weight \\
\hline \multirow[t]{2}{*}{ HWDXLM } & Tests if decay vertex lies in given volume \\
\hline & Elementary subprocess generation \\
\hline HWEFIN & Final calculations on elementary subprocess \\
\hline HWEGAM & Generates incoming photon \\
\hline HWEINI & Initializes elementary subprocess \\
\hline HWEISR & Generates photon emission from $e$ or $\mu$ \\
\hline HWEONE & Sets up a $2 \rightarrow 1$ hard subprocess \\
\hline HWEPRO & Generates elementary subprocess \\
\hline \multirow[t]{2}{*}{ HWETWO } & Sets up a $2 \rightarrow 2$ hard subprocess \\
\hline & Individual hard subprocesses \\
\hline HWH2BK & Matrix element for $b \bar{b} \rightarrow W^{ \pm} H^{\mp}$ \\
\hline HWH2QH & Matrix element for $q \bar{q}, g g \rightarrow Q \bar{Q}^{\left({ }^{\prime}\right)}$ Higgs \\
\hline
\end{tabular}

Table 20: Subroutines. (Continues) 


\begin{tabular}{|c|c|}
\hline Name & Description \\
\hline HWH2VH & Matrix element for $q \bar{q}^{\left({ }^{\prime}\right)} \rightarrow V$ Higgs, $V=W^{ \pm}, Z^{0}$ \\
\hline HWH4 J1 & Matrix element for $e^{+} e^{-} \rightarrow 4$ jets \\
\hline HWH4 JT & Hard subprocess: $e^{+} e^{-} \rightarrow 4$ jets \\
\hline HWHBGF & Hard subprocess: boson-gluon fusion (BGF) \\
\hline HWHBKI & Computes kinematics for BGF \\
\hline HWHBRN & Returns a phase-space point for BGF \\
\hline HWHBSG & Computes cross section for BGF \\
\hline HWHDIS & Hard subprocess: deep inelastic $e / \mu$ quark \\
\hline HWHDYP & Hard subprocess: Drell-Yan $Z^{0} / \gamma$ prodn \\
\hline HWHEGG & Hard subprocess: two-photon processes in $e^{+} e^{-}$ \\
\hline HWHEGW & Hard subprocess: $\gamma W$ processes in $e^{+} e^{-}$ \\
\hline HWHEGX & Calculates cross section for HWHEGW \\
\hline HWHEPA & Hard subprocess: $e^{+} e^{-} \rightarrow q \bar{q}$ \\
\hline HWHEPG & Hard subprocess: $e^{+} e^{-} \rightarrow q \bar{q} g$ \\
\hline HWHESG & Gaugino pair production in $e^{+} e^{-}$collisons \\
\hline HWHESL & Slepton pair production in $e^{+} e^{-}$collisons \\
\hline HWHESP & Sparticle pair production in $e^{+} e^{-}$collisons \\
\hline HWHESQ & Squark pair production in $e^{+} e^{-}$collisons \\
\hline HWHEWO & $e^{+} e^{-} \rightarrow W^{+} W^{-}$subroutine \\
\hline HWHEW1 & $e^{+} e^{-} \rightarrow W^{+} W^{-}$subroutine \\
\hline HWHEW2 & $e^{+} e^{-} \rightarrow W^{+} W^{-}$subroutine \\
\hline HWHEW3 & $e^{+} e^{-} \rightarrow W^{+} W^{-}$subroutine \\
\hline HWHEW4 & $e^{+} e^{-} \rightarrow W^{+} W^{-}$subroutine \\
\hline HWHEW5 & $e^{+} e^{-} \rightarrow W^{+} W^{-}$subroutine \\
\hline HWHEWW & Hard subprocess: $e^{+} e^{-} \rightarrow W^{+} W^{-}$ \\
\hline HWHGRV & Graviton resonance production \\
\hline HWHHVY & Hard subprocess: heavy quark production \\
\hline HWHIBG & Hard subprocess: for $b g \rightarrow Q$ Higgs, with $Q=t, b$ \\
\hline HWHIBK & Hard subprocess: $b \bar{b} \rightarrow W^{ \pm} H^{\mp}$ \\
\hline HWHIG1 & Matrix elements for Higgs + jet production \\
\hline HWHIGA & Amplitudes squared for Higgs + jet \\
\hline HWHIGB & Loop integrals for Higgs + jet \\
\hline HWHIGH & Hard subprocess: $q \bar{q} \rightarrow$ Higgs $_{1}+$ Higgs $_{2}$ \\
\hline HWHIGJ & QCD Higgs + jet production \\
\hline HWHIGM & Choose any Higgs mass for production routines \\
\hline HWHIGQ & Hard subprocess: $q \bar{q}, g g \rightarrow Q \bar{Q}^{\left({ }^{\prime}\right)}$ Higgs \\
\hline HWHIGS & Hard subprocess: $g g / q \bar{q} \rightarrow$ Higgs \\
\hline HWHIGT & Computes $g g \rightarrow$ Higgs cross section \\
\hline
\end{tabular}

Table 26: Subroutines. (Continues) 


\begin{tabular}{|c|c|}
\hline Name & Description \\
\hline HWHIGV & Hard subprocess: $q \bar{q}^{\left({ }^{\prime}\right)} \rightarrow V$ Higgs, $V=W^{ \pm}, Z^{0}$ \\
\hline HWHIGW & Hard subprocess: $W^{+} W^{-} / Z^{0} Z^{0} \rightarrow$ Higgs \\
\hline HWHIGY & Computes $e^{+} e^{-} \rightarrow Z^{0} \rightarrow Z^{0} H_{\mathrm{SM}}^{0}$ cross section \\
\hline HWHIGZ & Hard subprocess: $e^{+} e^{-} \rightarrow Z^{0} \rightarrow Z^{0} H_{\mathrm{SM}}^{0}$ \\
\hline HWHPH2 & Hard subprocess: direct photon pairs \\
\hline HWHPHO & Hard subprocess: direct photon production \\
\hline HWHPPB & Box contribution to $g g \rightarrow \gamma \gamma$ \\
\hline HWHPPE & Pointlike photon-parton (fixed flavour) \\
\hline HWHPPH & Pointlike photon-parton (fixed pair flavour) \\
\hline HWHPPM & Pointlike photon-parton direct light meson \\
\hline HWHPPT & Pointlike photon-parton (all flavours) \\
\hline HWHPQS & Pointlike photon-quark (Compton) scattering \\
\hline HWHQCD & QCD $2 \rightarrow 2$ hard subprocesses \\
\hline HWHQCP & Identifies QCD $2 \rightarrow 2$ hard subprocess \\
\hline HWHRBB & $R_{\mathrm{p}}$ resonant squark to SM particles \\
\hline HWHRBS & $R_{\mathrm{p}}$ resonant squark to SUSY particles \\
\hline HWHREE & $R_{\mathrm{p}} \mathrm{SM}$ particle production in $e^{+} e^{-}$collisions \\
\hline HWHREM & Treats hard scattering remnants \\
\hline HWHREP & Decides which $R_{\mathrm{p}}$ subroutine to use in $e^{+} e^{-}$ \\
\hline HWHRES & $R_{\mathrm{p}}$ single sparticle production in $e^{+} e^{-}$collisions \\
\hline HWHRLL & $R_{\mathrm{p}}$ resonant slepton to $\mathrm{SM}$ particles \\
\hline HWHRLS & $R_{\mathrm{p}}$ resonant slepton to SUSY particles \\
\hline HWHRSP & Decides which $R_{\mathrm{p}}$ subroutine to use in hadron-hadron \\
\hline HWHRSS & Identifies $R_{\mathrm{p}}$ process \\
\hline HWHSCT & Process extra hard scatterings \\
\hline HWHSNG & Colour singlet parton scattering \\
\hline HWHSNM & Colour singlet parton scattering matrix element \\
\hline HWHSS1 & Gaugino-gaugino production matrix element \\
\hline HWHSSG & Gaugino-gaugino/gaugino-sparton production \\
\hline HWHSSL & Slepton pair production \\
\hline HWHSSP & Combines MSSM subprocesses \\
\hline HWHSSQ & SQCD $2 \rightarrow 2$ hard subprocesses \\
\hline HWHSSS & Identifies MSSM hard subprocess \\
\hline HWHV1 J & Hard subprocess $\mathrm{W} / \mathrm{Z}+$ jet production \\
\hline HWHWEX & Top production by $\mathrm{W}$ exchange \\
\hline \multirow[t]{2}{*}{ HWHWPR } & Hard subprocess: W production \\
\hline & Soft minimum-bias or underlying event \\
\hline HWMEVT & $\begin{array}{c}\text { Generates min bias or soft underlying event } \\
\text { Table } \mathbf{\mathbf { 2 6 }} \text { : Subroutines. (Continues) }\end{array}$ \\
\hline
\end{tabular}




\begin{tabular}{|c|c|}
\hline Name & Description \\
\hline HWMLPS & Generates longitudinal phase space \\
\hline HWMNBI & Computes negative binomial probability \\
\hline HWMULT & Chooses min bias charged multiplicity \\
\hline \multirow[t]{2}{*}{ HWMWGT } & Calculates weight for minimum bias events \\
\hline & Random number generators \\
\hline HWRAZM & Randomly rotated azimuth \\
\hline HWREXP & Random number: exponential distribution \\
\hline HWREXQ & Random number: exp. dist. with cutoff \\
\hline HWREXT & Random number: exponential transverse mass \\
\hline HWRGAU & Random number: gaussian \\
\hline HWRGEN & Random number generator (l'Ecuyer's method) \\
\hline HWRINT & Random integer \\
\hline HWRLOG & Random logical \\
\hline HWRPIP & Random primary interaction point \\
\hline HWRPOW & Random number: power distribution \\
\hline HWRUNG & Random number: uniform + gaussian tails \\
\hline \multirow[t]{2}{*}{ HWRUNI } & Random number: uniform \\
\hline & Spacelike branching of incoming partons \\
\hline HWSBRN & Generates spacelike parton branching \\
\hline HWSDGG & Drees-Grassie gluon distribution in photon \\
\hline HWSDGQ & Drees-Grassie quark distribution in photon \\
\hline HWSFBR & Chooses a spacelike branching \\
\hline HWSFUN & Hadron structure functions \\
\hline HWSGAM & Gamma function (for structure functions) \\
\hline HWSGEN & Generates $x$ values for spacelike partons \\
\hline HWSGQQ & Inserts $g \rightarrow q \bar{q}$ part of gluon form factor \\
\hline HWSSPC & Replaces spacelike partons by spectators \\
\hline HWSSUD & Sudakov form factor/structure function \\
\hline HWSTAB & Interpolates in function table (for HWSSUD) \\
\hline \multirow[t]{2}{*}{ HWSVAL } & Checks for valence parton \\
\hline & Miscellaneous utilities \\
\hline HWUAEM & Running electromagnetic coupling constant \\
\hline HWUAER & Real part of photon self-energy \\
\hline HWUALF & Two-loop QCD running coupling constant \\
\hline HWUANT & Finds a particle's antiparticle \\
\hline HWUBPR & Prints branching data for last parton shower \\
\hline HWUBST & Boost event record to/from hadron-hadron c.m.f. \\
\hline HWUCFF & Coefficients for $e^{+} e^{-}$and DIS cross sections \\
\hline
\end{tabular}

Table 26: Subroutines. (Continues) 


\begin{tabular}{|l|l|}
\hline NAme & Description \\
\hline HWUCI2 & Logarithmic integral Ci 2 \\
HWUDAT & Particle properties (N.B. BLOCK DATA) \\
HWUDKL & Generates decay vertex of unstable particle \\
HWUDKM & Stores new decay modes \\
HWUDKS & Converts decay modes into internal format \\
HWUDPR & Prints particle properties and decay modes \\
HWUECM & Centre-of-mass energy \\
HWUEDT & Insert or delete entries in the event record \\
HWUEEC & Computes coefficients for $e^{+} e^{-}$cross section \\
HWUEMV & Moves entries within the event record \\
HWUEPH & Prints event headers \\
HWUEPR & Prints event data \\
HWUFNE & Finishes an event \\
HWUGAU & Adaptive gaussian integration \\
HWUIDT & Translates particle identity codes \\
HWUINC & Initial parameter-dependent calculations \\
HWUINE & Initializes an event \\
HWULB4 & Boost: rest frame to lab, no masses assumed \\
HWULDO & Lorentz 4-vector dot product \\
HWULF4 & Boost: lab frame to rest, no masses assumed \\
HWULI2 & Logarithmic integral Li ${ }_{2}$ Spence function) \\
HWULOB & Lorentz transformation: rest frame $\rightarrow$ lab \\
HWULOF & Lorentz transformation: lab $\rightarrow$ rest frame \\
HWULOR & Multiplies by Lorentz matrix \\
HWUTAB & HWUM
\end{tabular}

Table 26: Subroutines. (Continues) 


\begin{tabular}{|c|l|}
\hline Name & Description \\
\hline & Vector manipulation \\
\hline HWVDIF & Vector difference \\
HWVDOT & Vector dot product \\
HWVEQU & Vector equality \\
HWVSCA & Vector times scalar \\
HWVSUM & Vector sum \\
HWVZRI & Vector zero (integer) \\
HWVZRO & Vector zero \\
\hline HWWARN & Issues warnings and deals with errors \\
\hline
\end{tabular}

Table 늠: Subroutines.

In addition there are the routines for generating the Schuler-Sjöstrand parton distributions of the photon:

\section{SASANO SASBEH SASDIR SASGAM SASVMD}

Finally, there are dummy versions of external routines, which should be deleted if the relevant packages are used:

- CERN library function for time remaining (dummy sets this to $10^{5}$ seconds).

TIMEL

- CERN PDFLIB structure function package:

PDFSET STRUCTM

- EURODEC B decay package:

EUDINI FRAGMT IEUPDG IPDGEU

- CLEO B decay package:

DECADD QQINIT QQLMAT

- HERBVI baryon number violation package:

HVCBVI HVHBVI 


\section{Acknowledgments}

We thank G. Abbiendi, J. Chýla and L. Stanco for their collaboration on earlier versions of HERWIG and for their continuing interest. We also thank U. Baur, M.J. Gibbs, E.W.N. Glover, Z. Kunszt and D.R. Ward for contributing code to be incorporated into the program.

We are grateful to the many experimental colleagues who have made valuable criticisms and suggestions over the years, especially K. Hamacher, R. Hemingway and G. Rudolph.

\section{References}

[1] G. Marchesini, B.R. Webber, G. Abbiendi, I.G. Knowles, M. H. Seymour and L. Stanco, HERWIG: a Monte Carlo event generator for simulating hadron emission reactions with interfering gluons. Version 5.1 - april 1991, $\bar{C}_{\text {Comput. }} \bar{P} \bar{h} \bar{y}$ s. (-

[2] A. Bassetto, M. Ciafaloni and G. Marchesini, Jet structure and infrared sensitive

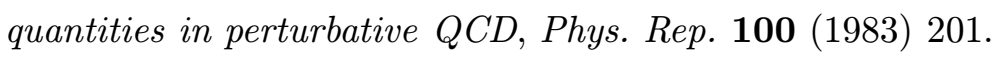

[3] G. Marchesini and B. R. Webber, Simulation of QCD jets including soft gluon inter-

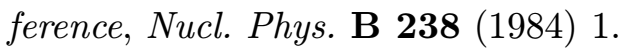

[4] B.R. Webber, A QCD model for jet fragmentation including soft gluon interference,

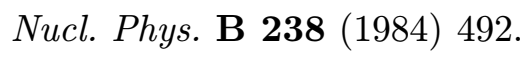

[5] B.R. Webber, Monte Carlo simulation of hard hadronic processes, iĀnn._Rev. Nucl.' :

[6] R.K. Ellis, G. Marchesini and B.R. Webber, Soft radiation in parton parton scattering, 'Nucl.

[7] G. Marchesini and B.R. Webber, Monte Carlo simulation of general hard processes with coherent $Q C D$ radiation, № $u c l$. Phys. B 310 $(1988)-461$.

[8] G. Marchesini and B.R. Webber, Associated transverse energy in hadronic jet production, 'Ṕ

[9] I.G. Knowles, Spin correlations in parton-parton scattering, 'Nučl. Phys. - - - - -

[10] I.G. Knowles, A linear algorithm for calculating spin correlations in hadronic colli-

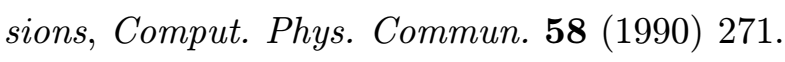

[11] G. Marchesini and B.R. Webber, Simulation of QCD coherence in heavy quark production and decay, iNucl. Phys. B 330 1990$)-261$ 
[12] G. Marchesini and B.R. Webber, Simulation of QCD initial state radiation at small

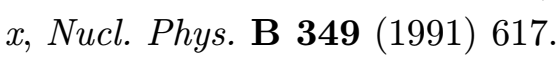

[13] S. Catani, B.R. Webber and G. Marchesini, QCD coherent branching and semiinclusive processes at large $x$, Nucl. Phys. B $\mathbf{3} 49$ (1991) 635 .

[14] G. Abbiendi and L. Stanco, Heavy flavor production at HERA. Simulation with a

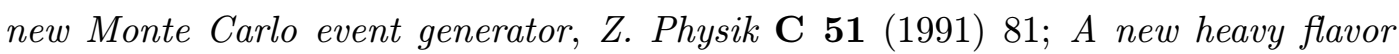
generator in e-p collisions, 'Comput. Phys. Commun.66 $19 \overline{1} \overline{1} 1 \overline{1}$.

[15] M.H. Seymour, Photon radiation in final state parton showering, 'ZZ Physik $\overline{\mathbf{C}}-\overline{\mathbf{5}} \overline{\mathbf{6}}$ :

[16] M.H. Seymour, Matrix element corrections to parton shower algorithms, 'Comput.: i.

[17] M.H. Seymour, A simple prescription for first order corrections to quark scattering

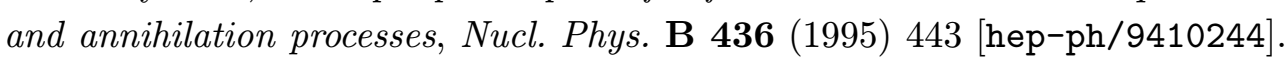

[18] M.H. Seymour, Matrix element corrections to parton shower simulation of deep inelastic scattering, contributed to 27th International Conference on High Energy Physics (ICHEP), Glasgow 1994, Lund preprint LU-TP-94-12, unpublished.

[19] G. Corcella and M.H. Seymour, Matrix element corrections to parton shower simu-

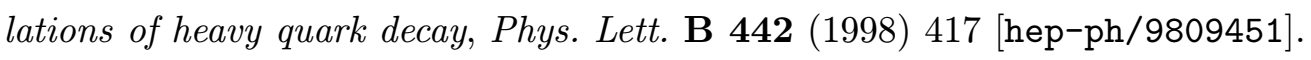

[20] G. Corcella, M.H. Seymour, Initial state radiation in simulations of vector boson

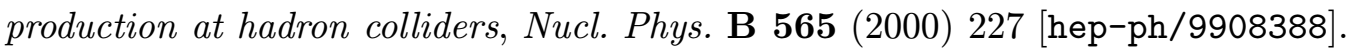

[21] S. Moretti, K. Odagiri, P. Richardson, M.H. Seymour and B.R. Webber, preprint Cavendish-HEP-98/06, in preparation.

[22] D. Amati and G. Veneziano, Preconfinement as a property of perturbative $Q C D$, P'Phys.' (- Lett. B 83. 1 1979) 87 .

[23] Yu.L. Dokshitzer and S.I. Troyan, Nonleading perturbative corrections to the dynamics of quark-gluon cascades and soft hadron spectra in $e^{+} e^{-}$annihilation, Leningrad Nuclear Physics Institute preprint N 922 (1984);

Y.I. Azimov, Yu.L. Dokshitzer, V.A. Khoze and S.I. Troian, The string effect and

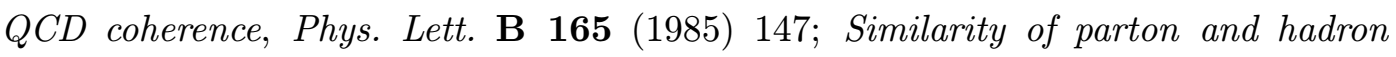
spectra in $Q C D$ jets, $\bar{Z}$.

[24] T. Sjöstrand et al., QCD event generators for LEP, in $Z$ Physics at LEP, G. Altarelli, R. Kleiss and C. Verzegnassi eds., CERN 89-08.

[25] I.G. Knowles, T. Sjöstrand et al. Report of QCD Event Generators Working Group, in Physics at LEP2, ed. G. Altarelli, T. Sjöstrand and F. Zwirner, CERN 96-01.

[26] Yu.L. Dokshitzer, V.A. Khoze, S.I. Troyan and A.H. Müller, QCD coherence in high-

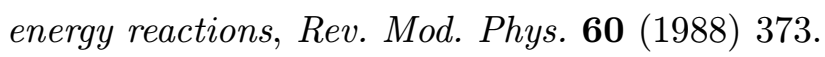




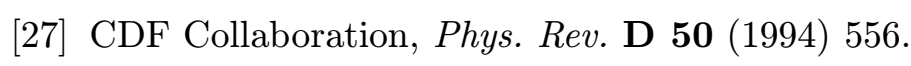

[28] D $\varnothing$ collaboration, Color coherent radiation in multijet events from ppbar collisions at $\sqrt{s}=1.8 \mathrm{TeV}$ 'P hyss. Lett. B $\mathbf{4} \mathbf{1} 4$ (1997) 419' [hep-ex/9706012];

Evidence of color coherence effects in $W^{+}$jets events from $p \bar{p}$ collisions at $\sqrt{s}=$

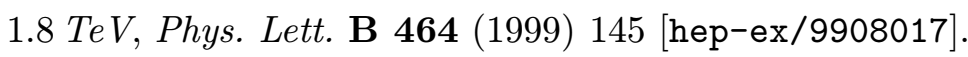

[29] K. Odagiri, Color connection structure of (supersymmetric) QCD (2 $\rightarrow$ 2) processes,

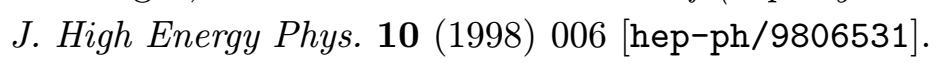

[30] Yu.L. Dokshitzer, V.A. Khoze and S.I. Troian, New perturbative results in hadron jet physics, in Proc. Physics in Collision 6, Chicago 1986, p.417.

[31] A. Einstein, B. Podolski and N. Rosen, Can quantum mechanical description of phys-

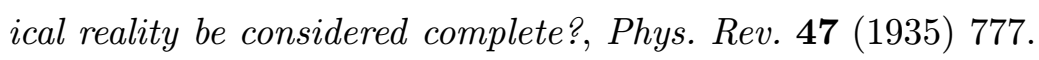

[32] G. Marchesini, QCD coherence in the structure function and associated distributions at small $x$, Nucl. Phys. B 445 (1995) 49 i [hep-ph/9412327i].

[33] T. Sjöstrand, High-energy physics event generation with PYTHIA 5.7 and JETSET 7.4, 'Comput. Phys. Commun. $\mathbf{8 2}(1994)$ 74.

[34] M.H. Seymour, The Higgs boson line shape and perturbative unitarity, . - . - 3

[35] D. E. Groom et al., Review of particle physics, 'Eur. Phys.

[36] F.E. Paige, S.D. Protopopescu, H. Baer and X. Tata, ISAJET 7.37: a Monte Carlo event generator for $p p, \bar{p} p$ and $e^{+} e^{-}$reactions, hhep-ph/9804321; ISAJET 7.40: a Monte Carlo event generator for $p p$, $\bar{p} p$, and $e^{+} e^{-}$reactions preprint BNL-HET98/39, FSU-HEP-981016, UH-511-917-98, October 1998, [hep-ph/9810440i]. Latest version available from 'fetp: //penguin.phy.bni.gov:/pub/isajet/l

[37] H.E. Haber and G.L. Kane, The search for supersymmetry: probing physics beyond the Standard Model, $\stackrel{\bar{P}}{\mathrm{P}} \overline{\mathrm{y}} \mathrm{s}$. $\mathrm{Re}$.

[38] J.F. Gunion, H. E. Haber, G.L. Kane and S. Dawson, The Higgs hunter guide, Addison-Wesley, Reading MA 1990.

[39] H. Dreiner, P. Richardson and M.H. Seymour, Parton-shower simulations of $R$ -

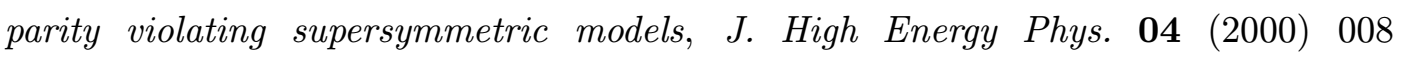
[hep-ph/9912407i].

[40] H. Dreiner, An introduction to explicit R-parity violation in Perspectives on Supersymmetry, G.L. Kane ed., World Scientific, pp. 462-479 [hep-ph/9707435i].

[41] M.J. Gibbs, A. Ringwald, B.R. Webber and J.T. Zadrozny, Monte Carlo simulation of baryon and lepton number violating processes at high-energies, 'IZ : 
M.J. Gibbs, B.R. Webber, HERBVI: a program for simulation of baryon and

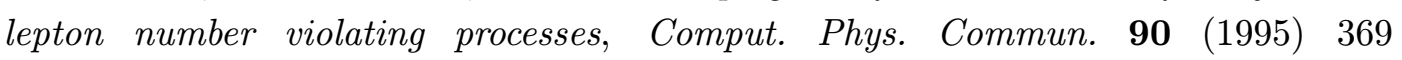
[hep-ph/9504232].

[42] A. Kupco, Cluster hadronization in HERWIG 5.9, in Proc. Workshop on Monte Carlo Generators for HERA Physics, Hamburg 1998, p.292 [hep-ph/9906412i].

[43] B. Andersson, G. Gustafsson, G. Ingelman and T. Sjöstrand, ํㅡㄴ -

[44] TPC/Two Gamma collaboration, H. Aihara et al., Study of baryon correlations in $e^{+} e^{-}$annihilation at $29 \mathrm{GeV},{ }^{\prime}$ Phys. Rev. Lett. $\mathbf{5} 7$ (1986)_3140.

[45] UA5 collaboration, G.J. Alner et al., The UA5 high-energy $\bar{p} p$ simulation program,

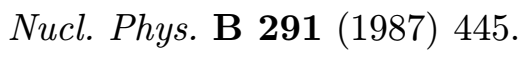

[46] J.M. Butterworth and J.R. Forshaw, Photoproduction of multi-jet events at HERA: a Monte Carlo simulation,

J.M. Butterworth, J.R. Forshaw and M.H. Seymour, Multiparton interactions in

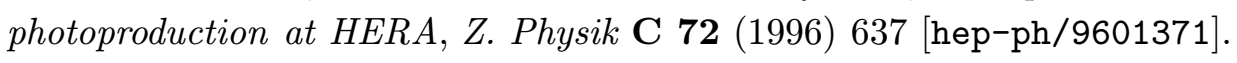

[47] H. Plothow-Besch, PDFLIB: a library of all available parton density functions of the nucleon, the pion and the photon and the corresponding alpha-s calculations, ${ }_{1} \bar{C}$ omput.'

:- Phys. Commun.

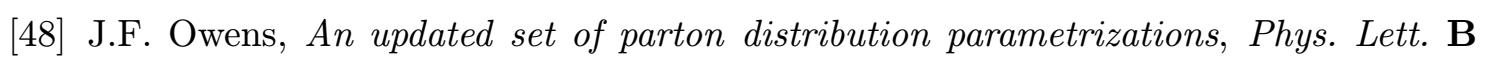
(-2)

[49] M. Drees and K. Grassie, Parametrizations of the photon structure and applications

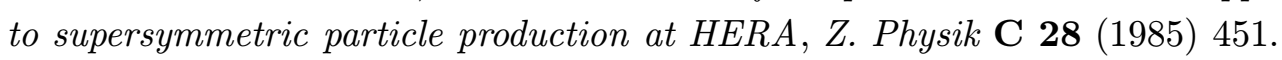

[50] M. Drees and C.S. Kim, Associate production of a heavy quark and a gauge boson at $e^{+} p$ colliders, 'ZZ

[51] G. A. Schuler and T. Sjostrand, Parton distributions of the virtual photon, 'Phys.' [

[52] M. Drees and R.M. Godbole, Virtual photon structure functions and the parton content of the electron, 'Phys. Rev. D 50 (1994)_3124i [hep-ph/9403229i'].

[53] Z Physics at LEP1, CERN 89-09, vol. 3, p. 129.

[54] H. Burkhardt, F. Jegerlehner, G. Penso and C. Verzegnassi, Uncertainties in the

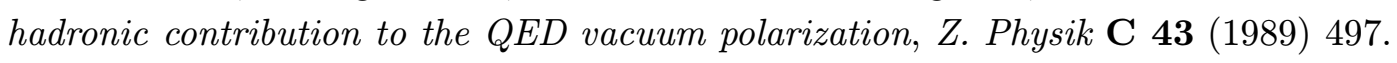

[55] R. K. Ellis, D. A. Ross and A. E. Terrano, The perturbative calculation of jet structure

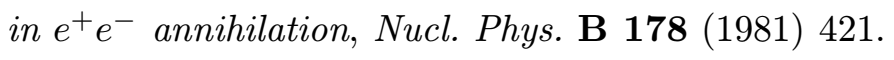


[56] S. Catani and M.H. Seymour, A general algorithm for calculating jet cross sections in NLO QCD, iNucl. Phys. B 485 (1997) 291 [hep-ph/9605323';

erratum ibid. $\mathbf{5 1 0}$ (1997) 503.

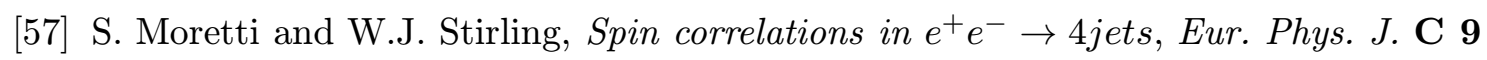
:

S. Moretti, The implementation of the four-jet matrix elements in HERWIG and elsewhere, preprint RAL-TR-2000-039, August 2000 [hep-ph/0008197i].

[58] S. Katsanevas and P. Morawitz, SUSYGEN 2.2: a Monte Carlo event generator for MSSM sparticle production at $e^{+} e^{-}$colliders, ${ }^{-}$Comput. Phys.Commun. $\mathbf{1} \mathbf{1} 2$ (1998), 227 [hep-ph/9711417i].

[59] J. Kalinowski, R. Ruckl, H. Spiesberger and P.M. Zerwas, Supersymmetry with Rparity breaking: contact interactions and resonance formation in leptonic processes at LEPQ, 'P

[60] A.H. Mueller and W.K. Tang, High-energy parton-parton elastic scattering in QCD,

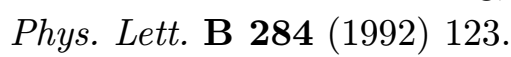

[61] E. Eichten, I. Hinchliffe, K. Lane and C. Quigg, Super collider physics, Reve. Mod: (1986) 1065.

[62] S. Dawson, E. Eichten and C. Quigg, Search for supersymmetric particles in hadronhadron collisions, 'Phys. Rev. D $\mathbf{3} 1$ (19.985) 15811 .

[63] A.A. Barrientos-Bendezù and B.A. Kniehl, $W^{ \pm} H^{\mp}$ associated production at the Large

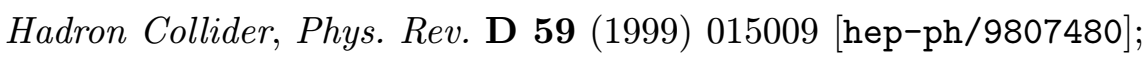

S. Moretti and K. Odagiri, The phenomenology of $W^{ \pm} H^{\mp}$ production at the Large

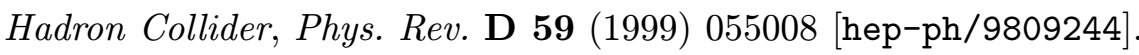

[64] ATLAS collaboration, Technical proposal, LHCC/P2 1994.

[65] CMS collaboration, Discovery potential for supersymmetry in CMS, Technical proposal, LHCC/P1 1994;

S. Abdullin et al., preprint TE 1998/06, June 1998, [hep-ph/9806366i].

[66] B.C. Allanach, K. Odagiri, M.A. Parker and B.R. Webber, Searching for narrow graviton resonances with the ATLAS detector at the large hadron collider, ' $\bar{J}-\bar{H} \bar{i} \bar{h} \bar{h}$

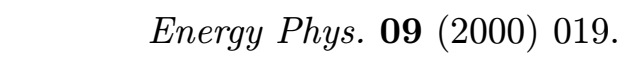

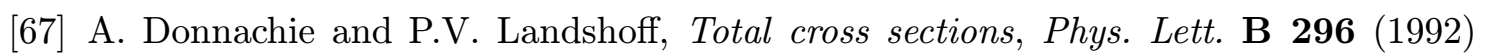
- 227 [ [hep-ph/9209205i].

[68] S. Moch, A. Ringwald and F. Schrempp, Instantons in deep-inelastic scattering. The simplest process, '슬. induced cross-sections in deep inelastic scattering, [hep-ph/9806528']. 
[69] M. Gibbs, A. Ringwald and F. Schrempp, QCD-instanton induced final states in deep inelastic scattering, in Proc. DIS 1995 (Paris), pp. 341-344 [hep-ph/9506392;

A. Ringwald and F. Schrempp, QCDINS 2.0:a Monte Carlo generator for instantoninduced processes in deep-inelastic scattering, to be published in Comp. Phys. Commun. [hep-ph/99911516]

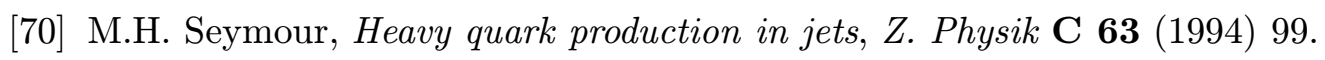

